\title{
Chiral extrapolations of baryon masses for unquenched three-flavor lattice simulations*
}

\author{
Matthias Frink \\ Institut für Kernphysik (Theorie), Forschungszentrum Jülich, D-52425 Jülich, Germany \\ and \\ Helmholtz-Institut für Strahlen- und Kernphysik (Theorie), Universität Bonn \\ Nußallee 14-16, D-53115 Bonn, Germany \\ E-mail: m.frink@fz-juelich.de
}

\author{
Ulf-G. Meißner \\ Helmholtz-Institut für Strahlen- und Kernphysik (Theorie), Universität Bonn \\ Nußallee 14-16, D-53115 Bonn, Germany \\ and \\ Institut für Kernphysik (Theorie), Forschungszentrum Jülich, D-52425 Jülich, Germany \\ E-mail: meissner@itkp.uni-bonn.de
}

\begin{abstract}
We have analyzed the chiral expansion of the baryon masses to second order in the quark masses, including strong isospin violation. The calculations are performed in Lorentz-invariant baryon chiral perturbation theory. Chiral extrapolation functions for three-flavor unquenched lattice simulations are derived. The matching to the two-flavor case and the sigma terms are also considered.
\end{abstract}

KEYwORDs: Chiral perturbation theory, baryon masses, lattice extrapolations.

${ }^{*}$ Work supported in part by funds provided from the EU to the project "Study of Strongly Interacting Matter" under contract no. RII3-CT-2004-506078. 


\section{Contents}

1. Introduction 1

2. Effective Lagrangian $\quad 2$

2.1 Meson Lagrangian 3

2.2 Meson-Baryon Lagrangian 5

3. Calculation of baryon masses $\quad 6$

4. Chiral extrapolation functions $\quad 9$

5. Matching to the two-flavor case 20

5.1 Matching equations 20

5.2 Bounds on the low-energy constants 24

6. Sigma terms $\quad 25$

$\begin{array}{ll}\text { 7. Summary and conclusions } & 27\end{array}$

A. Meson mass representation 28

B. Analysis of tapdole graphs 44

C. Renormalization and $\beta$-functions $\quad 46$

\section{Introduction}

Lattice QCD supplied with chiral perturbation theory is a powerful non-perturbative tool to analyze the structure of QCD in the confinement regime. It has been realized that lattice simulations performed at unphysically large quark masses can be extrapolated systematically to the physical limit based on functions derived in chiral perturbation theory or extensions thereof, see e.g. [1, 2]. Many observables like the pion mass, nucleon form factors and so on have been discussed in the literature, we focus here on the simplest baryonic two-point functions, namely the masses of the ground state octet. While there is still on-going debate about the precise implementation of such schemes in terms of regulator prescriptions and the corresponding resummation of classes of higher order terms (see e.g. the discussions in Refs. $[3,4,5,6]$ ), there is little controversy about the inherent usefulness of such studies. This is documented by the excellent fits one obtains for the quark mass expansion of the nucleon mass applied to two-flavor simulations, which typically have pion masses larger than $400 \mathrm{MeV}$, see e.g. [7, 8, 9]. It should also be pointed out that 
such two-flavor studies can be constrained by chiral perturbation theory analyses of pionnucleon scattering, since these allow one to pin down some combinations of the low-energy constants (LECs) appearing in the quark mass expansion of the nucleon mass. Recently, detailed finite size studies for the nucleon mass $\left(N_{F}=2\right)$ have also become available [10] 1 . Still, simulations at lower quark masses are needed to further sharpen these analyses.

The situation is entirely different in the three-flavor sector $\left(N_{F}=3\right)$. Only recently a large lattice effort was initiated to study tree-flavor dynamical QCD [12], and it will take some time before detailed results are available. In the meson sector, one has little doubt that chiral perturbation theory can be applied, although there are speculations of a strong flavor dependence of certain order parameters [13]. In the baryon sector, the issue of the convergence of the chiral expansion is not settled, but it is fair to say that more higher order calculations are needed for a final clarification ${ }^{2}$. Calculations of the nucleon (baryon) mass(es) to third and fourth order in various variants of chiral perturbation theory have been performed, see e.g. [15]-[27]. It is obvious that for reliable extrapolation functions for the baryon masses one has to go beyond the leading non-analytic terms $\sim m_{\text {quark }}^{3 / 2}$, because these do not even provide a reliable extrapolation function for two flavors. It is thus mandatory to consider at least the terms quadratic in the quark masses. This will be the topic of the present paper. In contrast to the earlier work [18], we perform the calculation in a Lorentz-invariant framework based on the so-called infrared regularization (IR) [25]. Furthermore, we also include all strong isospin breaking effects $\sim m_{u}-m_{d}$ up-to-and-including fourth order in the chiral expansion. Concerning the inclusion of the spin-3/2 decuplet, we follow exactly the lines of [5], were it is discussed how such effects can be absorbed in the values of certain LECs. We are well aware that present day lattice studies are far from reaching the required accuracy to resolve these fine effects, but we hope that the representations given here will become useful in the future.

The manuscript is organized as follows. The effective Lagrangian underlying our calculation is briefly presented in Section 2. The calculation of the baryon masses is outlined in Section 3 and the resulting quark mass expansions of the octet ground state masses are collected in Section 4. To constrain combinations of the appearing low-energy constants, we perform matching to the $\mathrm{SU}(2)$ case in Section 5. Sigma terms are discussed in Section 6. We briefly summarize in Section 7. The appendices contain the representation of the baryon masses in terms of the Goldstone boson masses and one-loop integrals, a general discussion of the quark mass dependence of tadpole diagrams, and the $\beta$-functions related to the renormalization of the one-loop graphs.

\section{Effective Lagrangian}

In this section, we collect the terms of the effective chiral Lagrangian underlying our calculation. We only display the terms relevant to the topic under investigation and keep

\footnotetext{
${ }^{1}$ Note that in this paper we will only be concerned with unquenched lattice QCD, for a study of nucleon properties in partially quenched QCD see [11] and references therein.

${ }^{2}$ For a recent discussion about the onset of the chiral regime, see e.g. [14].
} 
the discussion as brief as possible. We employ the standard power counting in which external momenta count as order $p$ (more precisely meant are meson four- and baryonthree momenta), whereas a quark mass insertion is booked as $\mathcal{O}\left(p^{2}\right)$. Here, $p$ denotes a genuine small parameter. Our calculation includes all terms up-to-and-including order $p^{4}$ from the effective Lagrangian (one-loop approximation). The chiral limit is defined by $m_{u}=m_{d}=m_{s}=0$, where $m_{u}\left(m_{d}, m_{s}\right)$ denotes the up (down, strange) quark mass. The basic fields in our effective Lagrangian are the eight pseudoscalar (Pseudo-)Goldstone bosons $\left(\pi^{ \pm}, \pi^{0}, K^{ \pm}, K^{0}, \bar{K}^{0}, \eta\right)$ chirally coupled to the octet of the spin- $1 / 2$ baryons. The effects of the spin-3/2 decuplet are included in the low-energy constants appearing at the various orders.

\subsection{Meson Lagrangian}

First, we consider the mesonic Lagrangian [28]. The current quark mass matrix $\mathcal{M}=$ $\operatorname{diag}\left(m_{u}, m_{d}, m_{s}\right)$ is contained in the external source $\chi=2 B(s(x)+i p(x)), s(x)=\mathcal{M}+\ldots$ and $B=|\langle 0|\bar{q} q| 0\rangle| / F_{\pi}^{2}$ measures the strength of the quark condensate in the chiral limit. To one-loop accuracy, the pertinent effective Lagrangian includes terms of chiral dimension two and four,

$$
\mathcal{L}_{\text {eff }}=\mathcal{L}^{(2)}+\mathcal{L}^{(4)}
$$

The relevant terms are given by

$$
\begin{gathered}
\mathcal{L}^{(2)}=\frac{F_{\pi}^{2}}{4} \operatorname{Tr}\left\{u_{\mu} u^{\mu}\right\}+\frac{F_{\pi}^{2}}{4} \operatorname{Tr}\left\{\chi_{+}\right\}, \\
\mathcal{L}^{(4)}=L_{1}\left[\operatorname{Tr}\left\{u_{\mu} u^{\mu}\right\}\right]^{2}+L_{2} \operatorname{Tr}\left\{u_{\mu} u_{\nu}\right\} \operatorname{Tr}\left\{u^{\mu} u^{\nu}\right\}+L_{3} \operatorname{Tr}\left\{u_{\mu} u^{\mu} u_{\nu} u^{\nu}\right\} \\
+L_{4} \operatorname{Tr}\left\{u_{\mu} u^{\mu}\right\} \operatorname{Tr}\left\{\chi_{+}\right\}+L_{5} \operatorname{Tr}\left\{u_{\mu} u^{\mu} \chi_{+}\right\}+L_{6}\left[\operatorname{Tr}\left\{\chi_{+}\right\}\right]^{2} \\
+L_{7}\left[\operatorname{Tr}\left\{\chi_{-}\right\}\right]^{2}+L_{8} \frac{1}{2} \operatorname{Tr}\left\{\chi_{+} \chi_{+}+\chi_{-} \chi_{-}\right\},
\end{gathered}
$$

with

$$
u_{\mu}=i u^{\dagger} \partial_{\mu} U u^{\dagger} \sim \mathcal{O}(p), \quad \chi_{ \pm}=u^{\dagger} \chi u^{\dagger} \pm u \chi^{\dagger} u \sim \mathcal{O}\left(p^{2}\right)
$$

Here, $F_{\pi}$ is the pseudoscalar decay constant (in the chiral limit) and the trace "Tr" refers to flavor space. The $L_{i}$ are low-energy constants, they are in general scale-dependent and absorb the infinities generated by the one-loop graphs. The pseudoscalar Goldstone fields $(\phi=\pi, K, \eta)$ are collected in the $3 \times 3$ unimodular, unitary matrix $U$,

$$
U(\phi)=u^{2}(\phi)=\exp \left\{i \phi / F_{\pi}\right\}
$$

with

$$
\phi=\sqrt{2}\left(\begin{array}{ccc}
\frac{1}{\sqrt{2}} \pi^{0}+\frac{1}{\sqrt{6}} \eta & \pi^{+} & K^{+} \\
\pi^{-} & -\frac{1}{\sqrt{2}} \pi^{0}+\frac{1}{\sqrt{6}} \eta & K^{0} \\
K^{-} & \bar{K}^{0} & -\frac{2}{\sqrt{6}} \eta
\end{array}\right)
$$


in terms of the physical fields without $\pi^{0}-\eta$ mixing. Under $\mathrm{SU}(3)_{L} \times \mathrm{SU}(3)_{R}, U$ transforms as $U \rightarrow U^{\prime}=L U R^{\dagger}$, with $L, R \in \mathrm{SU}(3)_{L, R}$. The neutral pion and the eta mix, the physical fields are related to the pure $\mathrm{SU}(3)$ components via

$$
\left(\begin{array}{c}
\pi^{0} \\
\eta
\end{array}\right)=\left(\begin{array}{cc}
\cos \varepsilon & \sin \varepsilon \\
-\sin \varepsilon & \cos \varepsilon
\end{array}\right)\left(\begin{array}{l}
\phi_{3} \\
\phi_{8}
\end{array}\right) .
$$

The (lowest order) mixing angle can be fixed from the condition that the second order expression for the meson self-energy is diagonal,

$$
\Sigma_{\pi^{0} \eta}^{(2)}=\Sigma_{\eta \pi^{0}}^{(2)}=0
$$

resulting in

$$
\tan 2 \varepsilon=\sqrt{3} \frac{m_{d}-m_{u}}{2 m_{s}-m_{u}-m_{d}}=\frac{\sqrt{3}}{2} \frac{m_{d}-m_{u}}{m_{s}-\hat{m}}
$$

where $\hat{m}=\left(m_{u}+m_{d}\right) / 2$ is the average light quark mass. Numerically, the mixing angle is quite small. Note that the effect of further mixing with the $\eta^{\prime}$ is contained in the numerical values of some of the low-energy constants. From this, one reads off the leading terms in the quark mass expansion of the meson masses (as denoted by the bar),

$$
\begin{aligned}
\bar{M}_{\pi^{+}}^{2} & =B\left(m_{u}+m_{d}\right)=2 B \hat{m}, \\
\bar{M}_{\pi^{0}}^{2} & =2 B\left[\frac{m_{u}+m_{d}+m_{s}}{3}+\frac{m_{u}+m_{d}-2 m_{s}}{6} \cos (2 \varepsilon)+\frac{\sqrt{3}}{6}\left(m_{u}-m_{d}\right) \sin (2 \varepsilon)\right] \\
& =2 B\left[\frac{2 \hat{m}+m_{s}}{3}+\frac{\hat{m}-m_{s}}{3} \frac{1}{\cos (2 \varepsilon)}\right], \\
\bar{M}_{K^{+}}^{2} & =B\left(m_{u}+m_{s}\right)=B\left[\hat{m}-\frac{1}{\sqrt{3}} \tan (2 \varepsilon)\left(m_{s}-\hat{m}\right)+m_{s}\right], \\
\bar{M}_{K^{0}}^{2} & =B\left(m_{d}+m_{s}\right)=B\left[\hat{m}+\frac{1}{\sqrt{3}} \tan (2 \varepsilon)\left(m_{s}-\hat{m}\right)+m_{s}\right], \\
\bar{M}_{\eta}^{2} & =2 B\left[\frac{m_{u}+m_{d}+m_{s}}{3}-\frac{m_{u}+m_{d}-2 m_{s}}{6} \cos (2 \varepsilon)-\frac{\sqrt{3}}{6}\left(m_{u}-m_{d}\right) \sin (2 \varepsilon)\right] \\
& =2 B\left[\frac{2 \hat{m}+m_{s}}{3}-\frac{\hat{m}-m_{s}}{3} \frac{1}{\cos (2 \varepsilon)}\right],
\end{aligned}
$$

and by CPT $M_{\pi^{-}}=M_{\pi^{+}}, M_{\bar{K}^{0}}=M_{K^{0}}$. The complete one-loop expressions for the $\pi^{+}$ and $K^{+}$are collected in Appendix A. Note that in the following we use the charged pion and kaon masses as our reference values. If we use Eqs. (2.10) together with the leading isospin violating corrections $\sim m_{u}-m_{d}$ and $\sim e^{2}$, where the latter are the electromagnetic effects, to relate the Goldstone boson to the the quark masses, we get $\varepsilon=1 \cdot 10^{-2}$. This justifies expanding all functions of $\varepsilon$ in powers of this small parameter to the appropriate order, as will be done in what follows. 


\subsection{Meson-Baryon Lagrangian}

The ground state baryons with $J^{P}=\frac{1}{2}^{+}$are collected in the $\mathrm{SU}(3)$ matrix

$$
B=\left(\begin{array}{ccc}
\frac{1}{\sqrt{2}} \Sigma^{0}+\frac{1}{\sqrt{6}} \Lambda & \Sigma^{+} & p \\
\Sigma^{-} & -\frac{1}{\sqrt{2}} \Sigma^{0}+\frac{1}{\sqrt{6}} \Lambda & n \\
\Xi^{-} & \Xi^{0} & -\frac{2}{\sqrt{6}} \Lambda
\end{array}\right)
$$

in terms of the physical fields without $\Lambda-\Sigma^{0}$ mixing. It turns out convenient to diagonalize the second order baryon self-energy contribution with the help of the lowest order mixing angle $\varepsilon$ in Eq. (2.9). Note that to higher order in the chiral expansion the concept of a unique $\Lambda-\Sigma^{0}$ mixing angle breaks down, which calls for the introduction of two different angles. Under chiral $\mathrm{SU}(3) \times \mathrm{SU}(3), B$ transforms as any matter field, $B \rightarrow B^{\prime}=K B K^{\dagger}$, with $K(U, L, R)$ the compensator field representing an element of the conserved subgroup $\mathrm{SU}(3)_{V}$. The corresponding effective meson-baryon Lagrangian contains terms of even and odd dimension,

$$
\mathcal{L}_{\text {eff }}=\mathcal{L}^{(1)}+\mathcal{L}^{(2)}+\mathcal{L}^{(3)}+\mathcal{L}^{(4)},
$$

to the accuracy required here. The form of the lowest order Lagrangian is standard,

$$
\mathcal{L}^{(1)}=\operatorname{Tr}\left\{\bar{B}\left(i \gamma^{\mu} D_{\mu}-m_{0}\right) B\right\}+\frac{D}{2} \operatorname{Tr}\left\{\bar{B} \gamma^{\mu} \gamma^{5}\left\{u_{\mu}, B\right\}\right\}+\frac{F}{2} \operatorname{Tr}\left\{\bar{B} \gamma^{\mu} \gamma^{5}\left[u_{\mu}, B\right]\right\},
$$

with $D \simeq 0.81$ and $F \simeq 0.46$ [29] the two axial-vector couplings. $D_{\mu}$ is the chiral covariant derivative. For our analysis it suffices to use $D_{\mu}=\partial_{\mu}$. Furthermore, $m_{0}$ is the average octet mass in the chiral limit. This large mass scale, which is of the order of the scale of chiral symmetry breaking, $\Lambda_{\chi} \simeq 4 \pi F_{\pi}$, requires special treatment as detailed below. At dimension two, we have considerably more terms (for the construction principles see $[30,31,32])$

$$
\begin{aligned}
\mathcal{L}^{(2)} & =b_{D} \operatorname{Tr}\left\{\bar{B}\left\{\chi_{+}, B\right\}\right\}+b_{F} \operatorname{Tr}\left\{\bar{B}\left[\chi_{+}, B\right]\right\}+b_{0} \operatorname{Tr}\{\bar{B} B\} \operatorname{Tr}\left\{\chi_{+}\right\} \\
& +b_{1} \operatorname{Tr}\left\{\bar{B}\left[u_{\mu},\left[u^{\mu}, B\right]\right]\right\}+b_{2} \operatorname{Tr}\left\{\bar{B}\left\{u_{\mu},\left[u^{\mu}, B\right]\right\}\right\} \\
& +b_{3} \operatorname{Tr}\left\{\bar{B}\left\{u_{\mu},\left\{u^{\mu}, B\right\}\right\}\right\}+b_{4} \operatorname{Tr}\{\bar{B} B\} \operatorname{Tr}\left\{u_{\mu} u^{\mu}\right\} \\
& +\frac{b_{5}}{2}\left(\operatorname{Tr}\left\{\bar{B} i \gamma^{\mu}\left[u_{\mu},\left[u_{\nu},\left[D^{\nu}, B\right]\right]\right]\right\}+\operatorname{Tr}\left\{\bar{B} i \gamma^{\mu}\left[D_{\nu},\left[u^{\nu},\left[u_{\mu}, B\right]\right]\right\}\right)\right. \\
& +\frac{b_{6}}{2}\left(\operatorname{Tr}\left\{\bar{B} i \gamma^{\mu}\left[u_{\mu},\left\{u_{\nu},\left[D^{\nu}, B\right]\right\}\right]\right\}+\operatorname{Tr}\left\{\bar{B} i \gamma^{\mu}\left[D_{\nu},\left\{u^{\nu},\left[u_{\mu}, B\right]\right\}\right]\right\}\right) \\
& +\frac{b_{7}}{2}\left(\operatorname{Tr}\left\{\bar{B} i \gamma^{\mu}\left\{u_{\mu},\left\{u_{\nu},\left[D^{\nu}, B\right]\right\}\right\}\right\}+\operatorname{Tr}\left\{\bar{B} i \gamma^{\mu}\left[D_{\nu},\left\{u^{\nu},\left\{u_{\mu}, B\right\}\right\}\right]\right\}\right) \\
& +b_{8} \operatorname{Tr}\left\{\bar{B} i \gamma^{\mu}\left[D_{\nu}, B\right]\right\} \operatorname{Tr}\left\{u_{\mu} u^{\nu}\right\} \\
+ & \frac{b_{9}}{2}\left(\operatorname{Tr}\left\{\bar{B} u_{\mu}\right\} i \gamma^{\mu} \operatorname{Tr}\left\{u_{\nu}\left[D^{\nu}, B\right]\right\}+\operatorname{Tr}\left\{\bar{B} u_{\nu}\right\} i \gamma^{\mu} \operatorname{Tr}\left\{u_{\mu}\left[D^{\nu}, B\right]\right\}\right. \\
& \left.+\operatorname{Tr}\left\{\bar{B}\left[D_{\nu}, u^{\nu}\right]\right\} i \gamma^{\mu} \operatorname{Tr}\left\{u_{\mu} B\right\}+\operatorname{Tr}\left\{\bar{B} u_{\nu}\right\} i \gamma^{\mu} \operatorname{Tr}\left\{\left[D^{\nu}, u_{\mu}\right] B\right\}\right) .
\end{aligned}
$$

The LECs $b_{i}(i=0, D, F, 1, \ldots, 4)$ have dimension mass ${ }^{-1}$, whereas the $b_{j}(j=5, \ldots, 9)$ have dimension mass ${ }^{-2}$. The first three of these terms $\sim b_{0, D, F}$ stick out since they 
parameterize the explicit symmetry breaking and contribute already at tree level to the masses and the corresponding sigma terms. All other terms only play a role in the fourth order loop graphs. Note that we have more terms than were given in [18]. There, all terms with one or two covariant derivatives were absorbed in the structures $\sim b_{1,2,3,4}$. This can be done as long as one works for a set of fixed quark masses. For our purpose, we need to retain all terms that lead to structures of different quark mass dependences. A more detailed discussion of this topic is relegated to App. B. We also note that the complete minimal meson-baryon Lagrangian can be found in Ref.[32]. We have no contribution from the dimension three terms, thus

$$
\mathcal{L}^{(3)}=0 \text {. }
$$

Finally, there are tree contributions from the dimension four Lagrangian (we have performed a relabeling of the last two terms as compared to [18])

$$
\begin{aligned}
\mathcal{L}^{(4)} & =d_{1} \operatorname{Tr}\left\{\bar{B}\left[\chi_{+},\left[\chi_{+}, B\right]\right]\right\}+d_{2} \operatorname{Tr}\left\{\bar{B}\left[\chi_{+},\left\{\chi_{+}, B\right\}\right]\right\} \\
& +d_{3} \operatorname{Tr}\left\{\bar{B}\left\{\chi_{+},\left\{\chi_{+}, B\right\}\right\}\right\}+d_{4} \operatorname{Tr}\left\{\bar{B} \chi_{+}\right\} \operatorname{Tr}\left\{\chi_{+} B\right\} \\
& +d_{5} \operatorname{Tr}\left\{\bar{B}\left[\chi_{+}, B\right]\right\} \operatorname{Tr}\left\{\chi_{+}\right\}+d_{6} \operatorname{Tr}\{\bar{B} B\} \operatorname{Tr}\left\{\chi_{+}\right\} \operatorname{Tr}\left\{\chi_{+}\right\} \\
& +d_{7} \operatorname{Tr}\{\bar{B} B\} \operatorname{Tr}\left\{\chi_{+}^{2}\right\} .
\end{aligned}
$$

The LECs $d_{i}$ are of dimension mass ${ }^{-3}$. They are the sum of an infinite part to absorb the infinities from the boson loops and a finite part, that is of relevance here. Whenever we write down any of these LECs, it refers to its finite (renormalized) value at the renormalization scale $\lambda=m_{0}$ (as discussed in more detail below). Note that some of the terms in Eq. (2.16) are quark mass renormalizations of some of the second order terms listed in Eq. (2.14), but for our purpose we have to keep these contributions separated since they lead to different quark mass dependences.

\section{Calculation of baryon masses}

To calculate the baryon masses in chiral perturbation theory, we consider the baryonic two-point functions

$$
\int d^{4} x e^{i p \cdot x}\left\langle 0\left|T\left\{B(x) \bar{B}^{\prime}(0)\right\}\right| 0\right\rangle=\left(\frac{i}{\not p-m_{0}-\Sigma}\right)_{B B^{\prime}},
$$

with $\Sigma(\not p)$ the self-energy. Furthermore, $B\left(B^{\prime}\right)$ stands for any member of the ground state octet, $B=p, n, \Lambda, \Sigma^{0}, \Sigma^{+}, \Sigma^{-}, \Xi^{0}, \Xi^{-}$. The matrix notation is necessary because of the $\Lambda-\Sigma^{0}$ mixing. The baryon mass at a given order in the chiral expansion can be expressed in terms of the corresponding self-energy at the baryon pole for the diagonal states as

$$
\begin{aligned}
& m_{B}^{(0)}=m_{0} \\
& m_{B}^{(2)}=\Sigma_{B B}^{(2)}\left(\not p=m_{0}\right) \\
& m_{B}^{(3)}=\Sigma_{B B}^{(3)}\left(\not p=m_{0}\right) \\
& m_{B}^{(4)}=\Sigma_{B B}^{(4)}\left(\not p=m_{0}\right)+\frac{\partial}{\partial \not p}\left(\Sigma_{B B}^{(3)}\left(\not p=m_{0}\right)\right) \Sigma_{B B}^{(2)}\left(\not p=m_{0}\right),
\end{aligned}
$$



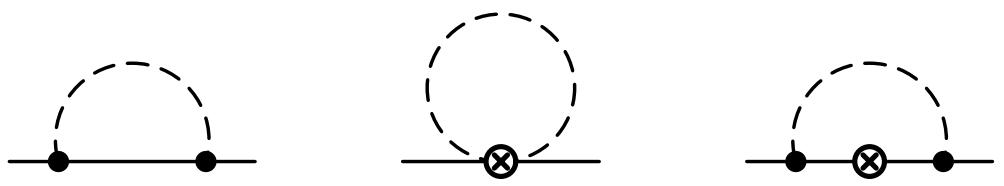

Figure 1: Loop contributions to the baryon masses at third and fourth order. Solid (dashed) lines refer to baryons (Goldstone bosons). The solid dot (circlecross) denotes an insertion from the dimension one (two) meson-baryon Lagrangian.

where the superscript $(n)$ denotes the chiral dimension. We remark that due to parity there are no first order contributions and $m_{0}$ denotes the octet mass in the chiral limit of vanishing up, down, and strange quark masses. For the $\Lambda$, one has

$$
\begin{aligned}
& m_{\Lambda}^{(0)}=m_{0}, \\
& m_{\Lambda}^{(2)}=\Sigma_{\Lambda \Lambda}^{(2)}\left(\not p=m_{0}\right) \\
& m_{\Lambda}^{(3)}=\Sigma_{\Lambda \Lambda}^{(3)}\left(\not p=m_{0}\right) \\
& m_{\Lambda}^{(4)}=\Sigma_{\Lambda \Lambda}^{(4)}\left(\not p=m_{0}\right)+\frac{\partial}{\partial \not p}\left(\Sigma_{\Lambda \Lambda}^{(3)}\left(\not p=m_{0}\right)\right) \Sigma_{\Lambda \Lambda}^{(2)}\left(\not p=m_{0}\right) \\
& \quad+\frac{\Sigma_{\Sigma^{0} \Lambda}^{(3)}\left(\not p=m_{0}\right) \Sigma_{\Lambda \Sigma^{0}}^{(3)}\left(\not p=m_{0}\right)}{\Sigma_{\Lambda \Lambda}^{(2)}\left(\not p=m_{0}\right)-\Sigma_{\Sigma^{0} \Sigma^{0}}^{(2)}\left(\not p=m_{0}\right)}
\end{aligned}
$$

and a similar expression holds for the $\Sigma^{0}$. It can be obtained from Eq. (3.3) by the interchange $\Lambda \leftrightarrow \Sigma^{0}$. The chiral expansion is equivalent to the quark mass expansion. The latter can symbolically be written as

$$
m_{B}=m_{0}+\sum_{q=u, d, s} A_{q}^{B} m_{q}+\sum_{q=u, d, s} B_{q}^{B} m_{q}^{3 / 2}+\sum_{q=u, d, s} C_{q}^{B} m_{q}^{2}+\ldots
$$

and the state-dependent coefficients $A_{q}^{B}, B_{q}^{B}$ and $C_{q}^{B}$ can be read off from Eqs. $(3.2,3.3)$ if one utilizes the relations between the Goldstone boson and the quark masses.

We now turn to the explicit calculation of the baryon masses. At first and second order, one only has contributions from tree graphs, whereas at third order one-loop and at fourth order tree and one-loop diagrams contribute. The relevant one-loop graphs are displayed in Fig. 1. We briefly discuss the structure of the related loop integrals. The finite baryon mass in the chiral limit spoils the one-to-one correspondence between the chiral and the loop expansion. There exist various methods to overcome this problem, we use here the socalled infrared regularization to separate the soft (chiral) loop contributions from the hard ones. The latter can be absorbed in local terms given by the effective chiral Lagrangian (for a more detailed discussion we refer to [25]). The essential trick in this method is to write a Feynman parameter representation for any loop integral and split the integration range $[0,1]$ as $[0, \infty]-[1, \infty]$. The first term defines the irregular part which contains all the interesting chiral physics and obeys the power counting. The regular part given by the second integral (which is generated by momenta of the size of the hard scale $m_{0}$ ) is a polynomial in external momenta and quark masses. This splitting is symmetry-preserving 
and can be applied to any loop graph. In what follows, the symbol $\int_{I}$ stands for the irregular part obtained by applying this procedure. As done in [25], we use dimensional regularization for separating the divergent parts and set $\lambda=m_{0}$, where $\lambda$ is the scale of dimensional regularization. In principle, the IR method generates some higher divergences, we follow the same strategy as in [25] and [33] and simply ignore these (a different approach is followed in [6]).

We now turn to the explicit representation of the relevant loop diagrams. The tadpole graph (one meson propagator) is standard

$$
\bar{\Delta}_{P}=\int_{I} \frac{d^{d} k}{(2 \pi)^{d}} \frac{i}{k^{2}-\bar{M}_{P}^{2}} \stackrel{d \rightarrow 4}{\longrightarrow} 2 \bar{M}_{P}^{2}\left(\bar{L}+\frac{1}{2} \bar{\mu}_{P}\right),
$$

with

$$
\begin{aligned}
\bar{\mu}_{P} & =\frac{1}{16 \pi^{2}} \ln \left(\frac{\bar{M}_{P}^{2}}{m_{0}^{2}}\right), \\
\bar{L} & =\frac{m_{0}^{d-4}}{16 \pi^{2}}\left[\frac{1}{d-4}+\frac{1}{2}\left(\gamma_{E}-1-\ln 4 \pi\right)\right],
\end{aligned}
$$

where $P$ stands for any Goldstone boson, $d$ denotes the number of space-time dimensions and $\gamma_{E}$ is the Euler-Mascheroni constant. This type of graph contributes only at fourth order since the tadpole with the lowest order $\bar{B} B P P$-vertex vanishes. The self-energy graph with lowest order insertions is given by (one meson and one nucleon propagator)

$$
I_{P}\left(q^{2}\right)=-\int_{I} \frac{d^{d} k}{(2 \pi)^{d}} \frac{i}{k^{2}-\bar{M}_{P}^{2}} \frac{1}{(q-k)^{2}-m_{0}^{2}} \stackrel{d \rightarrow 4}{\longrightarrow}-\frac{q^{2}-m_{0}^{2}+\bar{M}_{P}^{2}}{q^{2}} \bar{L}+\bar{I}_{P}\left(q^{2}\right) .
$$

This diagram gives the complete third order contribution. From the finite part, we only need the quark (meson) mass expansion at $q^{2}=m_{0}^{2}$,

$$
\bar{I}_{P}\left(m_{0}^{2}\right)=-\frac{1}{16 \pi} \frac{\bar{M}_{P}}{m_{0}}-\frac{1}{16 \pi^{2}} \frac{\bar{M}_{P}^{2}}{2 m_{0}^{2}}-\frac{\bar{M}_{P}^{2}}{2 m_{0}^{2}} \bar{\mu}_{P}+\mathcal{O}\left(p^{3}\right) .
$$

The first term $\sim \bar{M}_{P}$ in this integral generates the leading non-analytic terms in the quark mass expansion of the baryon masses. Similarly, the self-energy with one dimension two insertion (one meson and two nucleon propagators) can be expressed in terms of the loop function (note that we only need a special case of the corresponding function defined in [25])

$$
\tilde{I}_{P}^{12}\left(q^{2}\right)=\int_{I} \frac{d^{d} k}{(2 \pi)^{d}} \frac{i}{k^{2}-\bar{M}_{P}^{2}} \frac{1}{\left[(q-k)^{2}-m_{0}^{2}\right]^{2}} \stackrel{d \rightarrow 4}{\longrightarrow}-\frac{1}{q^{2}} \bar{L}+\bar{I}_{P}^{12}\left(q^{2}\right),
$$

and at $q^{2}=m_{0}^{2}$ the finite piece can be expanded as

$$
\bar{I}_{P}^{12}\left(m_{0}^{2}\right)=-\frac{1}{16 \pi^{2}} \frac{1}{2 m_{0}^{2}}-\frac{1}{2 m_{0}^{2}} \bar{\mu}_{P}+\mathcal{O}(p) .
$$

The third order self-energy contributions and the fourth order tadpoles with insertions $\sim b_{0, D, F}$ have already been evaluated in [26] in the isospin limit. In App. C, we discuss briefly the renormalization of the various loop contributions and collect the pertinent $\beta$ functions. 


\section{Chiral extrapolation functions}

This section contains the main part of this paper, namely the explicit expressions for the baryon masses in terms of the quark masses. We express all masses in terms of $\hat{m}, \varepsilon$ and $m_{s}$ and utilize the chiral limit value of $B$ throughout. To arrive at these results, we express the formulas for the baryon masses as functions of the meson masses given in App. A in terms of the quark masses. We only retain the linear terms in the parameter $\varepsilon$ that parameterizes isospin violation. Note that in the strict chiral counting, the second order terms from the effective meson-baryon Lagrangian also generate terms $\sim\left(m_{d}-m_{u}\right)^{2}$ to the accuracy we are working here. Since these terms are numerically irrelevant, we do not display them here (although we have calculated them). We now give the various terms proportional to $m_{q}, m_{q}^{3 / 2}$ and $m_{q}^{2}$ for the different baryons. The second order contributions $\sim m_{q}$ are

$$
m_{B}^{(2)}=\left(\gamma_{1, B}+\tilde{\gamma}_{1, B} \frac{\varepsilon}{\sqrt{3}}\right) B \hat{m}+\left(\gamma_{2, B}+\tilde{\gamma}_{2, B} \frac{\varepsilon}{\sqrt{3}}\right) B m_{s},
$$

where the $\gamma_{i, B}$ are the isospin-symmetric coefficients defined via

$$
\gamma_{i, \Sigma} \equiv \gamma_{i, \Sigma^{+}}=\gamma_{i, \Sigma^{-}}=\gamma_{i, \Sigma^{0}}, \quad \gamma_{i, N} \equiv \gamma_{i, p}=\gamma_{i, n}, \quad \gamma_{i, \Xi} \equiv \gamma_{i, \Xi^{-}}=\gamma_{i, \Xi^{0}} \quad(i=1,2) .
$$

These second order coefficients $\gamma_{B}$ are

$$
\begin{array}{ll}
\gamma_{1, \Sigma}=-8\left(b_{0}+b_{D}\right), & \gamma_{2, \Sigma}=-4 b_{0} \\
\gamma_{1, N}=-8 b_{0}-4\left(b_{D}+b_{F}\right), & \gamma_{2, N}=4\left(-b_{0}-b_{D}+b_{F}\right) \\
\gamma_{1, \Xi}=-8 b_{0}+4\left(-b_{D}+b_{F}\right), & \gamma_{2, \Xi}=-4\left(b_{0}+b_{D}+b_{F}\right) \\
\gamma_{1, \Lambda}=-8 b_{0}-\frac{8}{3} b_{D}, & \gamma_{2, \Lambda}=-4 b_{0}-\frac{16}{3} b_{D}
\end{array}
$$

in agreement with earlier calculations. The corresponding coefficients of the isospin breaking corrections $\sim \varepsilon$ are

$$
\begin{array}{ll}
\tilde{\gamma}_{1, \Sigma^{+}}=-16 b_{F}, & \tilde{\gamma}_{2, \Sigma^{+}}=16 b_{F}, \\
\tilde{\gamma}_{1, \Sigma^{-}}=16 b_{F}, & \tilde{\gamma}_{2, \Sigma^{-}}=-16 b_{F}, \\
\tilde{\gamma}_{1, p}=-8\left(b_{D}+b_{F}\right), & \tilde{\gamma}_{2, p}=8\left(b_{D}+b_{F}\right), \\
\tilde{\gamma}_{1, \Xi^{-}}=8\left(-b_{D}+b_{F}\right), & \tilde{\gamma}_{2, \Xi^{-}}=8\left(b_{D}-b_{F}\right), \\
\tilde{\gamma}_{1, n}=8\left(b_{D}+b_{F}\right), & \tilde{\gamma}_{2, n}=-8\left(b_{D}+b_{F}\right), \\
\tilde{\gamma}_{1, \Xi^{0}}=8\left(b_{D}-b_{F}\right), & \tilde{\gamma}_{2, \Xi^{0}}=8\left(-b_{D}+b_{F}\right) .
\end{array}
$$

Note that for the $\Lambda$ and $\Sigma^{0}$ the isospin breaking effects (and in particular the non-diagonal contributions in Eqs. (3.3)) are of order $\left(m_{u}-m_{d}\right)^{2}$. The third order contributions $\sim m_{q}^{3 / 2}$ can be most compactly written as

$$
\begin{aligned}
m_{B}^{(3)} & =\left(\delta_{1, B}+\tilde{\delta}_{1, B} \frac{\varepsilon}{\sqrt{3}}\right) \frac{\sqrt{2} B^{3 / 2}}{4 \pi F_{\pi}^{2}} \hat{m}^{3 / 2}+\delta_{2, B} \frac{B^{3 / 2}}{4 \pi F_{\pi}^{2}}\left(\hat{m}+m_{s}\right)^{3 / 2} \\
& +\left(\delta_{3, B}+\tilde{\delta}_{3, B} \frac{\varepsilon}{\sqrt{3}}\right) \frac{\sqrt{2} B^{3 / 2}}{4 \sqrt{3} \pi F_{\pi}^{2}}\left(\hat{m}+2 m_{s}\right)^{3 / 2}+\tilde{\delta}_{4, B} \frac{\varepsilon}{\sqrt{3}} \frac{B^{3 / 2}}{4 \pi F_{\pi}^{2}}\left(\hat{m}-m_{s}\right)\left(\hat{m}+m_{s}\right)^{1 / 2}
\end{aligned}
$$

where again we have isospin-symmetric coefficients,

$$
\delta_{i, \Sigma} \equiv \delta_{i, \Sigma^{+}}=\delta_{i, \Sigma^{-}}=\delta_{i, \Sigma^{0}}, \quad \delta_{i, N} \equiv \delta_{i, p}=\delta_{i, n}, \quad \delta_{i, \Xi} \equiv \delta_{i, \Xi^{-}}=\delta_{i, \Xi^{0}} \quad(i=1,2,3)
$$


which are explicitly given by

$$
\begin{array}{ll}
\delta_{1, \Sigma}=-\frac{1}{3} D^{2}-2 F^{2}, & \delta_{2, \Sigma}=-\frac{1}{2}\left(D^{2}+F^{2}\right), \\
\delta_{3, \Sigma}=-\frac{1}{9} D^{2}, & \delta_{1, N}=-\frac{3}{4} D^{2}-\frac{3}{2} D F-\frac{3}{4} F^{2}, \\
\delta_{2, N}=-\frac{5}{12} D^{2}+\frac{1}{2} D F-\frac{3}{4} F^{2}, & \delta_{3, N}=-\frac{1}{36} D^{2}+\frac{1}{6} D F-\frac{1}{4} F^{2} \\
\delta_{1, \Xi}=-\frac{3}{4} D^{2}+\frac{3}{2} D F-\frac{3}{4} F^{2}, & \delta_{2, \Xi}=-\frac{5}{12} D^{2}-\frac{1}{2} D F-\frac{3}{4} F^{2}, \\
\delta_{3, \Xi}=-\frac{1}{36} D^{2}-\frac{1}{6} D F-\frac{1}{4} F^{2}, & \delta_{1, \Lambda}=-D^{2}, \\
\delta_{2, \Lambda}=-\frac{1}{6} D^{2}-\frac{3}{2} F^{2}, & \delta_{3, \Lambda}=-\frac{1}{9} D^{2} .
\end{array}
$$

The coefficients of the third order isospin breaking $\varepsilon$-corrections are

$$
\begin{aligned}
& \tilde{\delta}_{1, \Sigma^{+}}=-2 D F, \quad \tilde{\delta}_{3, \Sigma^{+}}=\frac{2}{3} D F, \\
& \tilde{\delta}_{4, \Sigma^{+}}=-3 D F, \quad \tilde{\delta}_{1, \Sigma^{-}}=2 D F, \\
& \tilde{\delta}_{3, \Sigma^{-}}=-\frac{2}{3} D F, \quad \tilde{\delta}_{4, \Sigma^{-}}=3 D F, \\
& \tilde{\delta}_{1, p}=\frac{1}{2} D^{2}-D F-\frac{3}{2} F^{2}, \quad \tilde{\delta}_{3, p}=-\frac{1}{6} D^{2}+\frac{1}{3} D F+\frac{1}{2} F^{2} \text {, } \\
& \tilde{\delta}_{4, p}=\frac{1}{4} D^{2}-\frac{3}{2} D F-\frac{3}{4} F^{2}, \quad \tilde{\delta}_{1, \Xi^{-}}=\frac{1}{2} D^{2}+D F-\frac{3}{2} F^{2}, \\
& \tilde{\delta}_{3, \Xi^{-}}=-\frac{1}{6} D^{2}-\frac{1}{3} D F+\frac{1}{2} F^{2} \quad, \quad \tilde{\delta}_{4, \Xi^{-}}=\frac{1}{4} D^{2}+\frac{3}{2} D F-\frac{3}{4} F^{2} \text {, } \\
& \tilde{\delta}_{1, n}=-\frac{1}{2} D^{2}+D F+\frac{3}{2} F^{2}, \quad \tilde{\delta}_{3, n}=\frac{1}{6} D^{2}-\frac{1}{3} D F-\frac{1}{2} F^{2} \text {, } \\
& \tilde{\delta}_{4, n}=-\frac{1}{4} D^{2}+\frac{3}{2} D F+\frac{3}{4} F^{2}, \quad \tilde{\delta}_{1, \Xi^{0}}=-\frac{1}{2} D^{2}-D F+\frac{3}{2} F^{2}, \\
& \tilde{\delta}_{3, \Xi^{0}}=\frac{1}{6} D^{2}+\frac{1}{3} D F-\frac{1}{2} F^{2}, \quad \tilde{\delta}_{4, \Xi^{0}}=-\frac{1}{4} D^{2}-\frac{3}{2} D F+\frac{3}{4} F^{2} \text {. }
\end{aligned}
$$

Since these are the leading one-loop corrections, they only depend on the lowest order axial coupling constants $D$ and $F$. The major result of this investigation are the complete fourth order corrections $\sim m_{q}^{2}$. In terms of the quark masses, they can most conveniently be written as

$$
\begin{aligned}
m_{B}^{(4)} & =\left(\epsilon_{1, B}+\tilde{\epsilon}_{1, B} \frac{\varepsilon}{\sqrt{3}}\right) B^{2} \hat{m}^{2}+\left(\epsilon_{2, B}+\tilde{\epsilon}_{2, B} \frac{\varepsilon}{\sqrt{3}}\right) B^{2} m_{s}^{2}+\left(\epsilon_{3, B}+\tilde{\epsilon}_{3, B} \frac{\varepsilon}{\sqrt{3}}\right) B^{2} \hat{m} m_{s} \\
& +\left(\epsilon_{4, B}+\tilde{\epsilon}_{4, B} \frac{\varepsilon}{\sqrt{3}}\right) \frac{B^{2}}{16 \pi^{2} F_{\pi}^{2}} \hat{m}^{2} \ln \left(\frac{2 B \hat{m}}{m_{0}^{2}}\right) \\
& +\left(\epsilon_{5, B}+\tilde{\epsilon}_{5, B} \frac{\varepsilon}{\sqrt{3}}\right) \frac{B^{2}}{16 \pi^{2} F_{\pi}^{2}} \hat{m}^{2} \ln \left(\frac{B\left(\hat{m}+m_{s}\right)}{m_{0}^{2}}\right) \\
& +\left(\epsilon_{6, B}+\tilde{\epsilon}_{6, B} \frac{\varepsilon}{\sqrt{3}}\right) \frac{B^{2}}{16 \pi^{2} F_{\pi}^{2}} \hat{m}^{2} \ln \left(\frac{2 B\left(\hat{m}+2 m_{s}\right)}{3 m_{0}^{2}}\right) \\
& +\left(\epsilon_{7, B}+\tilde{\epsilon}_{7, B} \frac{\varepsilon}{\sqrt{3}}\right) \frac{B^{2}}{16 \pi^{2} F_{\pi}^{2}} m_{s}^{2} \ln \left(\frac{B\left(\hat{m}+m_{s}\right)}{m_{0}^{2}}\right) \\
& +\left(\epsilon_{8, B}+\tilde{\epsilon}_{8, B} \frac{\varepsilon}{\sqrt{3}}\right) \frac{B^{2}}{16 \pi^{2} F_{\pi}^{2}} m_{s}^{2} \ln \left(\frac{2 B\left(\hat{m}+2 m_{s}\right)}{3 m_{0}^{2}}\right) \\
& +\left(\epsilon_{9, B}+\tilde{\epsilon}_{9, B} \frac{\varepsilon}{\sqrt{3}}\right) \frac{B^{2}}{16 \pi^{2} F_{\pi}^{2}} \hat{m} m_{s} \ln \left(\frac{2 B \hat{m}}{m_{0}^{2}}\right) \\
& +\left(\epsilon_{10, B}+\tilde{\epsilon}_{10, B} \frac{\varepsilon}{\sqrt{3}}\right) \frac{B^{2}}{16 \pi^{2} F_{\pi}^{2}} \hat{m} m_{s} \ln \left(\frac{B\left(\hat{m}+m_{s}\right)}{m_{0}^{2}}\right) \\
& +\left(\epsilon_{11, B}+\tilde{\epsilon}_{11, B} \frac{\varepsilon}{\sqrt{3}}\right) \frac{B^{2}}{16 \pi^{2} F_{\pi}^{2}} \hat{m} m_{s} \ln \left(\frac{2 B\left(\hat{m}+2 m_{s}\right)}{3 m_{0}^{2}}\right)
\end{aligned}
$$


where again we defined isospin-symmetric coefficients as before

$$
\epsilon_{i, \Sigma} \equiv \epsilon_{i, \Sigma^{+}}=\epsilon_{i, \Sigma^{-}}=\epsilon_{i, \Sigma^{0}}, \quad \epsilon_{i, N} \equiv \epsilon_{i, p}=\epsilon_{i, n}, \quad \epsilon_{i, \Xi} \equiv \epsilon_{i, \Xi^{-}}=\epsilon_{i, \Xi^{0}} \quad(i=1, \ldots, 11)
$$

Note that to this order in $\varepsilon$, there are no terms $\sim m_{s}^{2} \ln \hat{m}$. The largest contributions stem indeed from the terms of order $m_{s}^{2}$ and $m_{s}^{2} \ln m_{s}$ at the physical quark masses, but in actual lattice simulations the up and down quarks might have a similar mass as the strange quark. It is therefore important to retain all these terms when connecting lattice results to the physical world.

The fourth order contributions $\epsilon_{B}$ read:

$$
\begin{aligned}
& \epsilon_{1, \Sigma}=\frac{1}{(4 \pi)^{2} F_{\pi}^{2}}\left(-\frac{67}{27} \frac{D^{2}}{m_{0}}-9 \frac{F^{2}}{m_{0}}+\left(\frac{136}{9} D^{2}+8 F^{2}\right) b_{D}+16 D F b_{F}-\frac{9}{2} m_{0} b_{5}\right. \\
& \left.-\frac{139}{54} m_{0} b_{7}-\frac{37}{9} m_{0} b_{8}-m_{0} b_{9}\right)-64\left(d_{3}+d_{6}\right)-32 d_{7} \text {, } \\
& \epsilon_{2, \Sigma}=\frac{1}{(4 \pi)^{2} F_{\pi}^{2}}\left(-\frac{43}{27} \frac{D^{2}}{m_{0}}-\frac{F^{2}}{m_{0}}-8\left(D^{2}+F^{2}\right) b_{D}-16 D F b_{F}-\frac{m_{0}}{2} b_{5}\right. \\
& \left.-\frac{43}{54} m_{0} b_{7}-\frac{13}{9} m_{0} b_{8}\right)-16\left(d_{6}+d_{7}\right) \\
& \epsilon_{3, \Sigma}=\frac{1}{(4 \pi)^{2} F_{\pi}^{2}}\left(-\frac{70}{27} \frac{D^{2}}{m_{0}}-2 \frac{F^{2}}{m_{0}}-\frac{64}{9} D^{2} b_{D}-m_{0} b_{5}-\frac{35}{27} m_{0} b_{7}-\frac{22}{9} m_{0} b_{8}\right)-64 d_{6}, \\
& \epsilon_{4, \Sigma}=-\frac{4}{3} \frac{D^{2}}{m_{0}}-8 \frac{F^{2}}{m_{0}}+24 b_{0}+\left(24+\frac{32}{3} D^{2}\right) b_{D}-32 b_{1}-16 b_{3}-24 b_{4}+8 m_{0} b_{5} \\
& +4 m_{0} b_{7}+6 m_{0} b_{8}+2 m_{0} b_{9} \\
& \epsilon_{5, \Sigma}=-\frac{D^{2}}{m_{0}}-\frac{F^{2}}{m_{0}}+8 b_{0}+\left(4+12\left(D^{2}+F^{2}\right)\right) b_{D}+24 D F b_{F}-4\left(b_{1}+b_{3}\right)-8 b_{4} \\
& +m_{0}\left(b_{5}+b_{7}\right)+2 m_{0} b_{8}, \\
& \epsilon_{6, \Sigma}=-\frac{4}{27} \frac{D^{2}}{m_{0}}+\frac{8}{9}\left(b_{0}+b_{D}\right)-\frac{16}{27} b_{3}-\frac{8}{9} b_{4}+\frac{4}{27} m_{0} b_{7}+\frac{2}{9} m_{0} b_{8}, \\
& \epsilon_{7, \Sigma}=-\frac{D^{2}}{m_{0}}-\frac{F^{2}}{m_{0}}+8 b_{0}+\left(4-12\left(D^{2}+F^{2}\right)\right) b_{D}-24 D F b_{F}-4\left(b_{1}+b_{3}\right)-8 b_{4} \\
& +m_{0}\left(b_{5}+b_{7}\right)+2 m_{0} b_{8}, \\
& \epsilon_{8, \Sigma}=-\frac{16}{27} \frac{D^{2}}{m_{0}}+\frac{32}{9} b_{0}-\frac{64}{27} b_{3}-\frac{32}{9} b_{4}+\frac{16}{27} m_{0} b_{7}+\frac{8}{9} m_{0} b_{8}, \\
& \epsilon_{9, \Sigma}=-\frac{32}{3} D^{2} b_{D} \\
& \epsilon_{10, \Sigma}=-2\left(\frac{D^{2}}{m_{0}}+\frac{F^{2}}{m_{0}}\right)+16 b_{0}+8\left(b_{D}-b_{1}-b_{3}\right)-16 b_{4}+2 m_{0}\left(b_{5}+b_{7}\right)+4 m_{0} b_{8}, \\
& \epsilon_{11, \Sigma}=-\frac{16}{27} \frac{D^{2}}{m_{0}}+\frac{32}{9} b_{0}+\frac{16}{9} b_{D}-\frac{64}{27} b_{3}-\frac{32}{9} b_{4}+\frac{16}{27} m_{0} b_{7}+\frac{8}{9} m_{0} b_{8},
\end{aligned}
$$




$$
\begin{aligned}
\epsilon_{1, N} & =\frac{1}{(4 \pi)^{2} F_{\pi}^{2}}\left(-\frac{209}{54} \frac{D^{2}}{m_{0}}-\frac{43}{9} \frac{D F}{m_{0}}-\frac{29}{6} \frac{F^{2}}{m_{0}}+\left(-\frac{52}{9} D^{2}+\frac{40}{3} D F-4 F^{2}\right) b_{D}\right. \\
& \left.+\left(\frac{20}{3} D^{2}-8 D F+12 F^{2}\right) b_{F}-\frac{29}{12} m_{0} b_{5}-\frac{43}{36} m_{0} b_{6}-\frac{245}{108} m_{0} b_{7}-\frac{37}{9} m_{0} b_{8}-\frac{m_{0}}{4} b_{9}\right) \\
& -16\left(d_{1}+d_{2}+d_{3}\right)-32 d_{5}-64 d_{6}-32 d_{7}, \\
\epsilon_{2, N} & =\frac{1}{(4 \pi)^{2} F_{\pi}^{2}}\left(-\frac{53}{54} \frac{D^{2}}{m_{0}}+\frac{17}{9} \frac{D F}{m_{0}}-\frac{17}{6} \frac{F^{2}}{m_{0}}+\left(\frac{52}{9} D^{2}-\frac{40}{3} D F+4 F^{2}\right) b_{D}\right. \\
& \left.+\left(-\frac{20}{3} D^{2}+8 D F-12 F^{2}\right) b_{F}-\frac{17}{12} m_{0} b_{5}+\frac{17}{36} m_{0} b_{6}-\frac{89}{108} m_{0} b_{7}-\frac{13}{9} m_{0} b_{8}-\frac{m_{0}}{4} b_{9}\right) \\
& +16\left(-d_{1}+d_{2}-d_{3}+d_{5}-d_{6}-d_{7}\right),
\end{aligned}
$$

$$
\begin{aligned}
\epsilon_{3, N} & =\frac{1}{(4 \pi)^{2} F_{\pi}^{2}}\left(-\frac{49}{27} \frac{D^{2}}{m_{0}}+\frac{26}{9} \frac{D F}{m_{0}}-\frac{13}{3} \frac{F^{2}}{m_{0}}-\frac{13}{6} m_{0} b_{5}+\frac{13}{18} m_{0} b_{6}-\frac{85}{54} m_{0} b_{7}\right. \\
& \left.-\frac{22}{9} m_{0} b_{8}-\frac{m_{0}}{2} b_{9}\right)+32\left(d_{1}-d_{3}\right)+16 d_{5}-64 d_{6} \\
\epsilon_{4, N} & =-3 \frac{D^{2}}{m_{0}}-6 \frac{D F}{m_{0}}-3 \frac{F^{2}}{m_{0}}+24 b_{0}+12\left(b_{D}+b_{F}-b_{1}-b_{2}-b_{3}\right)-24 b_{4} \\
& +3 m_{0}\left(b_{5}+b_{6}+b_{7}\right)+6 m_{0} b_{8}
\end{aligned}
$$

$$
\begin{aligned}
\epsilon_{5, N} & =-\frac{5}{6} \frac{D^{2}}{m_{0}}+\frac{D F}{m_{0}}-\frac{3}{2} \frac{F^{2}}{m_{0}}+8 b_{0}+\left(6-\frac{26}{3} D^{2}+20 D F-6 F^{2}\right) b_{D} \\
& +\left(-2+10 D^{2}-12 D F+18 F^{2}\right) b_{F}-6 b_{1}+2 b_{2}-6 b_{3}-8 b_{4}+\frac{3}{2} m_{0} b_{5}-\frac{m_{0}}{2} b_{6} \\
& +\frac{3}{2} m_{0} b_{7}+2 m_{0} b_{8}+\frac{m_{0}}{2} b_{9}, \\
\epsilon_{6, N} & =-\frac{1}{27} \frac{D^{2}}{m_{0}}+\frac{2}{9} \frac{D F}{m_{0}}-\frac{1}{3} \frac{F^{2}}{m_{0}}+\frac{8}{9} b_{0}+\frac{4}{9}\left(b_{D}+b_{F}\right)-\frac{4}{3} b_{1}+\frac{4}{9} b_{2}-\frac{4}{27} b_{3}-\frac{8}{9} b_{4} \\
& +\frac{m_{0}}{3} b_{5}-\frac{m_{0}}{9} b_{6}+\frac{m_{0}}{27} b_{7}+\frac{2}{9} m_{0} b_{8},
\end{aligned}
$$

$$
\begin{aligned}
\epsilon_{7, N} & =-\frac{5}{6} \frac{D^{2}}{m_{0}}+\frac{D F}{m_{0}}-\frac{3}{2} \frac{F^{2}}{m_{0}}+8 b_{0}+\left(6+\frac{26}{3} D^{2}-20 D F+6 F^{2}\right) b_{D} \\
& +\left(-2-10 D^{2}+12 D F-18 F^{2}\right) b_{F}-6 b_{1}+2 b_{2}-6 b_{3}-8 b_{4}+\frac{3}{2} m_{0} b_{5}-\frac{m_{0}}{2} b_{6} \\
& +\frac{3}{2} m_{0} b_{7}+2 m_{0} b_{8}+\frac{m_{0}}{2} b_{9}, \\
\epsilon_{8, N} & =-\frac{4}{27} \frac{D^{2}}{m_{0}}+\frac{8}{9} \frac{D F}{m_{0}}-\frac{4}{3} \frac{F^{2}}{m_{0}}+\frac{32}{9}\left(b_{0}+b_{D}-b_{F}\right)-\frac{16}{3} b_{1}+\frac{16}{9} b_{2}-\frac{16}{27} b_{3}-\frac{32}{9} b_{4} \\
& +\frac{4}{3} m_{0} b_{5}-\frac{4}{9} m_{0} b_{6}+\frac{4}{27} m_{0} b_{7}+\frac{8}{9} m_{0} b_{8},
\end{aligned}
$$




$$
\begin{aligned}
& \epsilon_{10, N}=-\frac{5}{3} \frac{D^{2}}{m_{0}}+2 \frac{D F}{m_{0}}-3 \frac{F^{2}}{m_{0}}+16 b_{0}+12 b_{D}-4 b_{F}-12 b_{1}+4 b_{2}-12 b_{3}-16 b_{4} \\
& +3 m_{0} b_{5}-m_{0} b_{6}+3 m_{0} b_{7}+4 m_{0} b_{8}+m_{0} b_{9} \\
& \epsilon_{11, N}=-\frac{4}{27} \frac{D^{2}}{m_{0}}+\frac{8}{9} \frac{D F}{m_{0}}-\frac{4}{3} \frac{F^{2}}{m_{0}}+\frac{32}{9} b_{0}+\frac{8}{3} b_{D}-\frac{8}{9} b_{F}-\frac{16}{3} b_{1}+\frac{16}{9} b_{2}-\frac{16}{27} b_{3}-\frac{32}{9} b_{4} \\
& +\frac{4}{3} m_{0} b_{5}-\frac{4}{9} m_{0} b_{6}+\frac{4}{27} m_{0} b_{7}+\frac{8}{9} m_{0} b_{8}, \\
& \epsilon_{1, \Xi}=\frac{1}{(4 \pi)^{2} F_{\pi}^{2}}\left(-\frac{209}{54} \frac{D^{2}}{m_{0}}+\frac{43}{9} \frac{D F}{m_{0}}-\frac{29}{6} \frac{F^{2}}{m_{0}}-\left(\frac{52}{9} D^{2}+\frac{40}{3} D F+4 F^{2}\right) b_{D}\right. \\
& \left.-\left(\frac{20}{3} D^{2}+8 D F+12 F^{2}\right) b_{F}-\frac{29}{12} m_{0} b_{5}+\frac{43}{36} m_{0} b_{6}-\frac{245}{108} m_{0} b_{7}-\frac{37}{9} m_{0} b_{8}-\frac{m_{0}}{4} b_{9}\right) \\
& +16\left(-d_{1}+d_{2}-d_{3}\right)+32 d_{5}-64 d_{6}-32 d_{7}, \\
& \epsilon_{2, \Xi}=\frac{1}{(4 \pi)^{2} F_{\pi}^{2}}\left(-\frac{53}{54} \frac{D^{2}}{m_{0}}-\frac{17}{9} \frac{D F}{m_{0}}-\frac{17}{6} \frac{F^{2}}{m_{0}}+\left(\frac{52}{9} D^{2}+\frac{40}{3} D F+4 F^{2}\right) b_{D}\right. \\
& \left.+\left(\frac{20}{3} D^{2}+8 D F+12 F^{2}\right) b_{F}-\frac{17}{12} m_{0} b_{5}-\frac{17}{36} m_{0} b_{6}-\frac{89}{108} m_{0} b_{7}-\frac{13}{9} m_{0} b_{8}-\frac{m_{0}}{4} b_{9}\right) \\
& -16\left(d_{1}+d_{2}+d_{3}+d_{5}+d_{6}+d_{7}\right) \text {, } \\
& \epsilon_{3, \Xi}=\frac{1}{(4 \pi)^{2} F_{\pi}^{2}}\left(-\frac{49}{27} \frac{D^{2}}{m_{0}}-\frac{26}{9} \frac{D F}{m_{0}}-\frac{13}{3} \frac{F^{2}}{m_{0}}-\frac{13}{6} m_{0} b_{5}-\frac{13}{18} m_{0} b_{6}-\frac{85}{54} m_{0} b_{7}\right. \\
& \left.-\frac{22}{9} m_{0} b_{8}-\frac{m_{0}}{2} b_{9}\right)+32\left(d_{1}-d_{3}\right)-16 d_{5}-64 d_{6} \\
& \epsilon_{4, \Xi}=-3 \frac{D^{2}}{m_{0}}+6 \frac{D F}{m_{0}}-3 \frac{F^{2}}{m_{0}}+24 b_{0}+12\left(b_{D}-b_{F}-b_{1}+b_{2}-b_{3}\right)-24 b_{4} \\
& +3 m_{0}\left(b_{5}-b_{6}+b_{7}\right)+6 m_{0} b_{8} \\
& \epsilon_{5, \Xi}=-\frac{5}{6} \frac{D^{2}}{m_{0}}-\frac{D F}{m_{0}}-\frac{3}{2} \frac{F^{2}}{m_{0}}+8 b_{0}+\left(6-\frac{26}{3} D^{2}-20 D F-6 F^{2}\right) b_{D} \\
& +\left(2-10 D^{2}-12 D F-18 F^{2}\right) b_{F}-6 b_{1}-2 b_{2}-6 b_{3}-8 b_{4}+\frac{3}{2} m_{0} b_{5}+\frac{m_{0}}{2} b_{6} \\
& +\frac{3}{2} m_{0} b_{7}+2 m_{0} b_{8}+\frac{m_{0}}{2} b_{9} \\
& \epsilon_{6, \Xi}=-\frac{1}{27} \frac{D^{2}}{m_{0}}-\frac{2}{9} \frac{D F}{m_{0}}-\frac{1}{3} \frac{F^{2}}{m_{0}}+\frac{8}{9} b_{0}+\frac{4}{9}\left(b_{D}-b_{F}\right)-\frac{4}{3} b_{1}-\frac{4}{9} b_{2}-\frac{4}{27} b_{3}-\frac{8}{9} b_{4} \\
& +\frac{m_{0}}{3} b_{5}+\frac{m_{0}}{9} b_{6}+\frac{m_{0}}{27} b_{7}+\frac{2}{9} m_{0} b_{8} \\
& \epsilon_{7, \Xi}=-\frac{5}{6} \frac{D^{2}}{m_{0}}-\frac{D F}{m_{0}}-\frac{3}{2} \frac{F^{2}}{m_{0}}+8 b_{0}+\left(6+\frac{26}{3} D^{2}+20 D F+6 F^{2}\right) b_{D} \\
& +\left(2+10 D^{2}+12 D F+18 F^{2}\right) b_{F}-6 b_{1}-2 b_{2}-6 b_{3}-8 b_{4}+\frac{3}{2} m_{0} b_{5}+\frac{m_{0}}{2} b_{6} \\
& +\frac{3}{2} m_{0} b_{7}+2 m_{0} b_{8}+\frac{m_{0}}{2} b_{9},
\end{aligned}
$$




$$
\begin{aligned}
& \epsilon_{8, \Xi}=-\frac{4}{27} \frac{D^{2}}{m_{0}}-\frac{8}{9} \frac{D F}{m_{0}}-\frac{4}{3} \frac{F^{2}}{m_{0}}+\frac{32}{9}\left(b_{0}+b_{D}+b_{F}\right)-\frac{16}{3} b_{1}-\frac{16}{9} b_{2}-\frac{16}{27} b_{3}-\frac{32}{9} b_{4} \\
& +\frac{4}{3} m_{0} b_{5}+\frac{4}{9} m_{0} b_{6}+\frac{4}{27} m_{0} b_{7}+\frac{8}{9} m_{0} b_{8}, \\
& \epsilon_{10, \Xi}=-\frac{5}{3} \frac{D^{2}}{m_{0}}-2 \frac{D F}{m_{0}}-3 \frac{F^{2}}{m_{0}}+16 b_{0}+12 b_{D}+4 b_{F}-12 b_{1}-4 b_{2}-12 b_{3}-16 b_{4} \\
& +3 m_{0} b_{5}+m_{0} b_{6}+3 m_{0} b_{7}+4 m_{0} b_{8}+m_{0} b_{9}, \\
& \epsilon_{11, \Xi}=-\frac{4}{27} \frac{D^{2}}{m_{0}}-\frac{8}{9} \frac{D F}{m_{0}}-\frac{4}{3} \frac{F^{2}}{m_{0}}+\frac{32}{9} b_{0}+\frac{8}{3} b_{D}+\frac{8}{9} b_{F}-\frac{16}{3} b_{1}-\frac{16}{9} b_{2}-\frac{16}{27} b_{3}-\frac{32}{9} b_{4} \\
& +\frac{4}{3} m_{0} b_{5}+\frac{4}{9} m_{0} b_{6}+\frac{4}{27} m_{0} b_{7}+\frac{8}{9} m_{0} b_{8}, \\
& \epsilon_{1, \Lambda}=\frac{1}{(4 \pi)^{2} F_{\pi}^{2}}\left(-\frac{121}{27} \frac{D^{2}}{m_{0}}-3 \frac{F^{2}}{m_{0}}-\left(\frac{200}{9} D^{2}+8 F^{2}\right) b_{D}-16 D F b_{F}-\frac{3}{2} m_{0} b_{5}\right. \\
& \left.-\frac{43}{18} m_{0} b_{7}-\frac{37}{9} m_{0} b_{8}-\frac{m_{0}}{9} b_{9}\right)-\frac{64}{3} d_{3}-\frac{32}{3} d_{4}-64 d_{6}-32 d_{7} \\
& \epsilon_{2, \Lambda}=\frac{1}{(4 \pi)^{2} F_{\pi}^{2}}\left(-\frac{25}{27} \frac{D^{2}}{m_{0}}-3 \frac{F^{2}}{m_{0}}+\left(\frac{8}{9} D^{2}+8 F^{2}\right) b_{D}+16 D F b_{F}-\frac{3}{2} m_{0} b_{5}\right. \\
& \left.-\frac{19}{18} m_{0} b_{7}-\frac{13}{9} m_{0} b_{8}-\frac{4}{9} m_{0} b_{9}\right)-\frac{128}{3} d_{3}-\frac{32}{3} d_{4}-16\left(d_{6}+d_{7}\right) \text {, } \\
& \epsilon_{3, \Lambda}=\frac{1}{(4 \pi)^{2} F_{\pi}^{2}}\left(-\frac{34}{27} \frac{D^{2}}{m_{0}}-6 \frac{F^{2}}{m_{0}}+\frac{64}{3} D^{2} b_{D}-3 m_{0} b_{5}-\frac{11}{9} m_{0} b_{7}-\frac{22}{9} m_{0} b_{8}-\frac{4}{9} m_{0} b_{9}\right) \\
& +\frac{64}{3} d_{4}-64 d_{6} \\
& \epsilon_{4, \Lambda}=-4 \frac{D^{2}}{m_{0}}+24 b_{0}+\left(8-32 D^{2}\right) b_{D}-16 b_{3}-24 b_{4}+4 m_{0} b_{7}+6 m_{0} b_{8}, \\
& \epsilon_{5, \Lambda}=-\frac{1}{3} \frac{D^{2}}{m_{0}}-3 \frac{F^{2}}{m_{0}}+8 b_{0}+\left(\frac{20}{3}-\frac{4}{3} D^{2}-12 F^{2}\right) b_{D}-24 D F b_{F}-12 b_{1}-\frac{4}{3} b_{3}-8 b_{4} \\
& +3 m_{0} b_{5}+\frac{m_{0}}{3} b_{7}+2 m_{0} b_{8} \\
& \epsilon_{6, \Lambda}=-\frac{4}{27} \frac{D^{2}}{m_{0}}+\frac{8}{9} b_{0}+\frac{8}{27} b_{D}-\frac{16}{9} b_{3}-\frac{8}{9} b_{4}+\frac{4}{9} m_{0} b_{7}+\frac{2}{9} m_{0}\left(b_{8}+b_{9}\right), \\
& \epsilon_{7, \Lambda}=-\frac{1}{3} \frac{D^{2}}{m_{0}}-3 \frac{F^{2}}{m_{0}}+8 b_{0}+\left(\frac{20}{3}+\frac{4}{3} D^{2}+12 F^{2}\right) b_{D}+24 D F b_{F}-12 b_{1}-\frac{4}{3} b_{3}-8 b_{4} \\
& +3 m_{0} b_{5}+\frac{m_{0}}{3} b_{7}+2 m_{0} b_{8} \\
& \epsilon_{8, \Lambda}=-\frac{16}{27} \frac{D^{2}}{m_{0}}+\frac{32}{9} b_{0}+\frac{128}{27} b_{D}-\frac{64}{9} b_{3}-\frac{32}{9} b_{4}+\frac{16}{9} m_{0} b_{7}+\frac{8}{9} m_{0}\left(b_{8}+b_{9}\right), \\
& \epsilon_{9, \Lambda}=32 D^{2} b_{D} \\
& \epsilon_{10, \Lambda}=-\frac{2}{3} \frac{D^{2}}{m_{0}}-6 \frac{F^{2}}{m_{0}}+16 b_{0}+\frac{40}{3} b_{D}-24 b_{1}-\frac{8}{3} b_{3}-16 b_{4}+6 m_{0} b_{5}+\frac{2}{3} m_{0} b_{7}+4 m_{0} b_{8}, \\
& \epsilon_{11, \Lambda}=-\frac{16}{27} \frac{D^{2}}{m_{0}}+\frac{32}{9} b_{0}+\frac{80}{27} b_{D}-\frac{64}{9} b_{3}-\frac{32}{9} b_{4}+\frac{16}{9} m_{0} b_{7}+\frac{8}{9} m_{0}\left(b_{8}+b_{9}\right) .
\end{aligned}
$$


The corresponding $\varepsilon$-coefficients are given by

$$
\begin{aligned}
& \tilde{\epsilon}_{1, \Sigma^{+}}=\frac{1}{(4 \pi)^{2} F_{\pi}^{2}}\left(-\frac{172}{9} \frac{D F}{m_{0}}+\frac{464}{3} D F b_{D}+\left(8+\frac{232}{3} D^{2}+120 F^{2}\right) b_{F}\right. \\
& \left.-8 b_{2}-\frac{16}{9} m_{0} b_{6}\right)-128\left(d_{2}+d_{5}\right) \\
& \tilde{\epsilon}_{2, \Sigma^{+}}=\frac{1}{(4 \pi)^{2} F_{\pi}^{2}}\left(\frac{140}{9} \frac{D F}{m_{0}}+48 D F b_{D}+\left(-8+24\left(D^{2}+F^{2}\right)\right) b_{F}+8 b_{2}\right. \\
& \left.+\frac{8}{9} m_{0} b_{6}\right)+64 d_{5} \\
& \tilde{\epsilon}_{3, \Sigma^{+}}=\frac{1}{(4 \pi)^{2} F_{\pi}^{2}}\left(\frac{32}{9} \frac{D F}{m_{0}}-\frac{608}{3} D F b_{D}-\left(\frac{304}{3} D^{2}+144 F^{2}\right) b_{F}+\frac{8}{9} m_{0} b_{6}\right) \\
& +128 d_{2}+64 d_{5} \\
& \tilde{\epsilon}_{4, \Sigma^{+}}=-8 \frac{D F}{m_{0}}+64 D F b_{D}+\left(32\left(1+D^{2}\right)+96 F^{2}\right) b_{F}-16 b_{2}+4 m_{0} b_{6}, \\
& \tilde{\epsilon}_{5, \Sigma^{+}}=-8 \frac{D F}{m_{0}}+96 D F b_{D}+\left(16+48\left(D^{2}+F^{2}\right)\right) b_{F}-16 b_{2}+4 m_{0} b_{6}, \\
& \tilde{\epsilon}_{6, \Sigma^{+}}=\frac{8}{9} \frac{D F}{m_{0}}-\frac{32}{9} b_{F}+\frac{16}{9} b_{2}-\frac{4}{9} m_{0} b_{6} \\
& \tilde{\epsilon}_{7, \Sigma^{+}}=8 \frac{D F}{m_{0}}+16\left(-b_{F}+b_{2}\right)-4 m_{0} b_{6}, \\
& \tilde{\epsilon}_{8, \Sigma^{+}}=\frac{32}{9}\left(\frac{D F}{m_{0}}-b_{F}\right)+\frac{64}{9} b_{2}-\frac{16}{9} m_{0} b_{6}, \\
& \tilde{\epsilon}_{9, \Sigma^{+}}=-64 D F b_{D}-\left(16+32 D^{2}+96 F^{2}\right) b_{F}, \\
& \tilde{\epsilon}_{10, \Sigma^{+}}=-96 D F b_{D}-48\left(D^{2}+F^{2}\right) b_{F} \text {, } \\
& \tilde{\epsilon}_{11, \Sigma^{+}}=\frac{32}{9} \frac{D F}{m_{0}}-\frac{80}{9} b_{F}+\frac{64}{9} b_{2}-\frac{16}{9} m_{0} b_{6}, \\
& \tilde{\epsilon}_{1, \Sigma^{-}}=\frac{1}{(4 \pi)^{2} F_{\pi}^{2}}\left(\frac{172}{9} \frac{D F}{m_{0}}-\frac{464}{3} D F b_{D}-\left(8+\frac{232}{3} D^{2}+120 F^{2}\right) b_{F}\right. \\
& \left.+8 b_{2}+\frac{16}{9} m_{0} b_{6}\right)+128\left(d_{2}+d_{5}\right) \text {, } \\
& \tilde{\epsilon}_{2, \Sigma^{-}}=\frac{1}{(4 \pi)^{2} F_{\pi}^{2}}\left(-\frac{140}{9} \frac{D F}{m_{0}}-48 D F b_{D}+\left(8-24\left(D^{2}+F^{2}\right)\right) b_{F}-8 b_{2}\right. \\
& \left.-\frac{8}{9} m_{0} b_{6}\right)-64 d_{5} \\
& \tilde{\epsilon}_{3, \Sigma^{-}}=\frac{1}{(4 \pi)^{2} F_{\pi}^{2}}\left(-\frac{32}{9} \frac{D F}{m_{0}}+\frac{608}{3} D F b_{D}+\left(\frac{304}{3} D^{2}+144 F^{2}\right) b_{F}-\frac{8}{9} m_{0} b_{6}\right) \\
& -128 d_{2}-64 d_{5} \text {, } \\
& \tilde{\epsilon}_{4, \Sigma^{-}}=8 \frac{D F}{m_{0}}-64 D F b_{D}-\left(32\left(1+D^{2}\right)+96 F^{2}\right) b_{F}+16 b_{2}-4 m_{0} b_{6},
\end{aligned}
$$




$$
\begin{aligned}
& \tilde{\epsilon}_{5, \Sigma^{-}}=8 \frac{D F}{m_{0}}-96 D F b_{D}-\left(16+48\left(D^{2}+F^{2}\right)\right) b_{F}+16 b_{2}-4 m_{0} b_{6}, \\
& \tilde{\epsilon}_{6, \Sigma^{-}}=-\frac{8}{9} \frac{D F}{m_{0}}+\frac{32}{9} b_{F}-\frac{16}{9} b_{2}+\frac{4}{9} m_{0} b_{6}, \\
& \tilde{\epsilon}_{7, \Sigma^{-}}=-8 \frac{D F}{m_{0}}+16\left(b_{F}-b_{2}\right)+4 m_{0} b_{6}, \\
& \tilde{\epsilon}_{8, \Sigma^{-}}=\frac{32}{9}\left(-\frac{D F}{m_{0}}+b_{F}\right)-\frac{64}{9} b_{2}+\frac{16}{9} m_{0} b_{6},
\end{aligned}
$$

$$
\begin{aligned}
\tilde{\epsilon}_{9, \Sigma^{-}} & =64 D F b_{D}+\left(16+32 D^{2}+96 F^{2}\right) b_{F}, \\
\tilde{\epsilon}_{10, \Sigma^{-}} & =96 D F b_{D}+48\left(D^{2}+F^{2}\right) b_{F}, \\
\tilde{\epsilon}_{11, \Sigma^{-}} & =-\frac{32}{9} \frac{D F}{m_{0}}+\frac{80}{9} b_{F}-\frac{64}{9} b_{2}+\frac{16}{9} m_{0} b_{6},
\end{aligned}
$$

$$
\begin{aligned}
\tilde{\epsilon}_{1, p} & =\frac{1}{(4 \pi)^{2} F_{\pi}^{2}}\left(\frac{25}{9} \frac{D^{2}}{m_{0}}-\frac{86}{9} \frac{D F}{m_{0}}-\frac{25}{3} \frac{F^{2}}{m_{0}}+\left(4+\frac{556}{9} D^{2}+\frac{136}{3} D F+60 F^{2}\right) b_{D}\right. \\
& +\left(4+\frac{68}{3} D^{2}+120 D F+60 F^{2}\right) b_{F}-4\left(b_{1}+b_{2}+b_{3}\right)-\frac{8}{3} m_{0} b_{5} \\
& \left.+\frac{8}{9} m_{0}\left(-b_{6}+b_{7}\right)\right)-64\left(d_{1}+d_{2}+d_{3}+d_{5}\right), \\
\tilde{\epsilon}_{2, p} & =\frac{1}{(4 \pi)^{2} F_{\pi}^{2}}\left(-\frac{17}{9} \frac{D^{2}}{m_{0}}+\frac{70}{9} \frac{D F}{m_{0}}+\frac{17}{3} \frac{F^{2}}{m_{0}}+\left(-4-\frac{68}{9} D^{2}-8 D F+12 F^{2}\right) b_{D}\right. \\
& +\left(-4\left(1+D^{2}\right)+24 D F+12 F^{2}\right) b_{F}+4\left(b_{1}+b_{2}+b_{3}\right)+\frac{4}{3} m_{0} b_{5} \\
& \left.+\frac{4}{9} m_{0}\left(b_{6}-b_{7}\right)\right)+64\left(-d_{1}+d_{3}\right)+32 d_{5},
\end{aligned}
$$

$$
\begin{aligned}
\tilde{\epsilon}_{3, p} & =\frac{1}{(4 \pi)^{2} F_{\pi}^{2}}\left(-\frac{8}{9} \frac{D^{2}}{m_{0}}+\frac{16}{9} \frac{D F}{m_{0}}+\frac{8}{3} \frac{F^{2}}{m_{0}}-\left(\frac{488}{9} D^{2}+\frac{112}{3} D F+72 F^{2}\right) b_{D}\right. \\
& \left.-\left(\frac{56}{3} D^{2}+144 D F+72 F^{2}\right) b_{F}+\frac{4}{3} m_{0} b_{5}+\frac{4}{9} m_{0}\left(b_{6}-b_{7}\right)\right)+128 d_{1} \\
& +64 d_{2}+32 d_{5}, \\
\tilde{\epsilon}_{4, p} & =2 \frac{D^{2}}{m_{0}}-4 \frac{D F}{m_{0}}-6 \frac{F^{2}}{m_{0}}+\left(16+48 D^{2}+96 D F+48 F^{2}\right)\left(b_{D}+b_{F}\right)-24 b_{1} \\
& +8\left(-b_{2}+b_{3}\right)+6 m_{0} b_{5}+2 m_{0}\left(b_{6}-b_{7}\right),
\end{aligned}
$$




$$
\begin{aligned}
& \tilde{\epsilon}_{5, p}=\frac{2}{3} \frac{D^{2}}{m_{0}}-4 \frac{D F}{m_{0}}-2 \frac{F^{2}}{m_{0}}+\left(8+\frac{104}{3} D^{2}-16 D F+24 F^{2}\right) b_{D} \\
& +\left(8\left(1-D^{2}\right)+48 D F+24 F^{2}\right) b_{F}-8\left(b_{1}+b_{2}+b_{3}\right)+2 m_{0}\left(b_{5}+b_{6}+b_{7}+b_{9}\right), \\
& \tilde{\epsilon}_{6, p}=-\frac{2}{9} \frac{D^{2}}{m_{0}}+\frac{4}{9} \frac{D F}{m_{0}}+\frac{2}{3} \frac{F^{2}}{m_{0}}-\frac{16}{9}\left(b_{D}+b_{F}\right)+\frac{8}{3} b_{1}+\frac{8}{9}\left(b_{2}-b_{3}\right)-\frac{2}{3} m_{0} b_{5} \\
& +\frac{2}{9} m_{0}\left(-b_{6}+b_{7}\right) \\
& \tilde{\epsilon}_{7, p}=-\frac{2}{3} \frac{D^{2}}{m_{0}}+4 \frac{D F}{m_{0}}+2 \frac{F^{2}}{m_{0}}-\left(8+\frac{64}{3} D^{2}\right) b_{D}+8\left(-b_{F}+b_{1}+b_{2}+b_{3}\right) \\
& -2 m_{0}\left(b_{5}+b_{6}+b_{7}+b_{9}\right) \\
& \tilde{\epsilon}_{8, p}=-\frac{8}{9} \frac{D^{2}}{m_{0}}+\frac{16}{9} \frac{D F}{m_{0}}+\frac{8}{3} \frac{F^{2}}{m_{0}}-\frac{16}{9}\left(b_{D}+b_{F}\right)+\frac{32}{3} b_{1}+\frac{32}{9}\left(b_{2}-b_{3}\right)-\frac{8}{3} m_{0} b_{5} \\
& +\frac{8}{9} m_{0}\left(-b_{6}+b_{7}\right) \\
& \tilde{\epsilon}_{9, p}=-\left(8+48 D^{2}+96 D F+48 F^{2}\right)\left(b_{D}+b_{F}\right) \text {, } \\
& \tilde{\epsilon}_{10, p}=\left(-\frac{40}{3} D^{2}+16 D F-24 F^{2}\right) b_{D}+\left(8 D^{2}-48 D F-24 F^{2}\right) b_{F}, \\
& \tilde{\epsilon}_{11, p}=-\frac{8}{9} \frac{D^{2}}{m_{0}}+\frac{16}{9} \frac{D F}{m_{0}}+\frac{8}{3} \frac{F^{2}}{m_{0}}-\frac{40}{9}\left(b_{D}+b_{F}\right)+\frac{32}{3} b_{1}+\frac{32}{9}\left(b_{2}-b_{3}\right)-\frac{8}{3} m_{0} b_{5} \\
& +\frac{8}{9} m_{0}\left(-b_{6}+b_{7}\right) \\
& \tilde{\epsilon}_{1, \Xi^{-}}=\frac{1}{(4 \pi)^{2} F_{\pi}^{2}}\left(\frac{25}{9} \frac{D^{2}}{m_{0}}+\frac{86}{9} \frac{D F}{m_{0}}-\frac{25}{3} \frac{F^{2}}{m_{0}}+\left(4+\frac{556}{9} D^{2}-\frac{136}{3} D F+60 F^{2}\right) b_{D}\right. \\
& +\left(-4-\frac{68}{3} D^{2}+120 D F-60 F^{2}\right) b_{F}+4\left(-b_{1}+b_{2}-b_{3}\right)-\frac{8}{3} m_{0} b_{5} \\
& \left.+\frac{8}{9} m_{0}\left(b_{6}+b_{7}\right)\right)+64\left(-d_{1}+d_{2}-d_{3}+d_{5}\right), \\
& \tilde{\epsilon}_{2, \Xi^{-}}=\frac{1}{(4 \pi)^{2} F_{\pi}^{2}}\left(-\frac{17}{9} \frac{D^{2}}{m_{0}}-\frac{70}{9} \frac{D F}{m_{0}}+\frac{17}{3} \frac{F^{2}}{m_{0}}+\left(-4-\frac{68}{9} D^{2}+8 D F+12 F^{2}\right) b_{D}\right. \\
& +\left(4\left(1+D^{2}\right)+24 D F-12 F^{2}\right) b_{F}+4\left(b_{1}-b_{2}+b_{3}\right)+\frac{4}{3} m_{0} b_{5} \\
& \left.-\frac{4}{9} m_{0}\left(b_{6}+b_{7}\right)\right)+64\left(-d_{1}+d_{3}\right)-32 d_{5} \text {, } \\
& \tilde{\epsilon}_{3, \Xi^{-}}=\frac{1}{(4 \pi)^{2} F_{\pi}^{2}}\left(-\frac{8}{9} \frac{D^{2}}{m_{0}}-\frac{16}{9} \frac{D F}{m_{0}}+\frac{8}{3} \frac{F^{2}}{m_{0}}+\left(-\frac{488}{9} D^{2}+\frac{112}{3} D F-72 F^{2}\right) b_{D}\right. \\
& \left.+\left(\frac{56}{3} D^{2}-144 D F+72 F^{2}\right) b_{F}+\frac{4}{3} m_{0} b_{5}-\frac{4}{9} m_{0}\left(b_{6}+b_{7}\right)\right)+128 d_{1} \\
& -64 d_{2}-32 d_{5} \\
& \tilde{\epsilon}_{4, \Xi^{-}}=2 \frac{D^{2}}{m_{0}}+4 \frac{D F}{m_{0}}-6 \frac{F^{2}}{m_{0}}+\left(16+48 D^{2}-96 D F+48 F^{2}\right)\left(b_{D}-b_{F}\right)-24 b_{1} \\
& +8\left(b_{2}+b_{3}\right)+6 m_{0} b_{5}-2 m_{0}\left(b_{6}+b_{7}\right),
\end{aligned}
$$




$$
\begin{aligned}
& \tilde{\epsilon}_{5, \Xi^{-}}=\frac{2}{3} \frac{D^{2}}{m_{0}}+4 \frac{D F}{m_{0}}-2 \frac{F^{2}}{m_{0}}+\left(8+\frac{104}{3} D^{2}+16 D F+24 F^{2}\right) b_{D} \\
& +\left(8\left(-1+D^{2}\right)+48 D F-24 F^{2}\right) b_{F}+8\left(-b_{1}+b_{2}-b_{3}\right)+2 m_{0}\left(b_{5}-b_{6}+b_{7}+b_{9}\right) \text {, } \\
& \tilde{\epsilon}_{6, \Xi^{-}}=-\frac{2}{9} \frac{D^{2}}{m_{0}}-\frac{4}{9} \frac{D F}{m_{0}}+\frac{2}{3} \frac{F^{2}}{m_{0}}+\frac{16}{9}\left(-b_{D}+b_{F}\right)+\frac{8}{3} b_{1}-\frac{8}{9}\left(b_{2}+b_{3}\right)-\frac{2}{3} m_{0} b_{5} \\
& +\frac{2}{9} m_{0}\left(b_{6}+b_{7}\right) \\
& \tilde{\epsilon}_{7, \Xi^{-}}=-\frac{2}{3} \frac{D^{2}}{m_{0}}-4 \frac{D F}{m_{0}}+2 \frac{F^{2}}{m_{0}}-\left(8+\frac{64}{3} D^{2}\right) b_{D}+8\left(b_{F}+b_{1}-b_{2}+b_{3}\right) \\
& +2 m_{0}\left(-b_{5}+b_{6}-b_{7}-b_{9}\right) \text {, } \\
& \tilde{\epsilon}_{8, \Xi^{-}}=-\frac{8}{9} \frac{D^{2}}{m_{0}}-\frac{16}{9} \frac{D F}{m_{0}}+\frac{8}{3} \frac{F^{2}}{m_{0}}+\frac{16}{9}\left(-b_{D}+b_{F}\right)+\frac{32}{3} b_{1}-\frac{32}{9}\left(b_{2}+b_{3}\right)-\frac{8}{3} m_{0} b_{5} \\
& +\frac{8}{9} m_{0}\left(b_{6}+b_{7}\right) \\
& \tilde{\epsilon}_{9, \Xi^{-}}=\left(-8-48 D^{2}+96 D F-48 F^{2}\right)\left(b_{D}-b_{F}\right), \\
& \tilde{\epsilon}_{10, \Xi^{-}}=-\left(\frac{40}{3} D^{2}+16 D F+24 F^{2}\right) b_{D}+\left(-8 D^{2}-48 D F+24 F^{2}\right) b_{F}, \\
& \tilde{\epsilon}_{11, \Xi^{-}}=-\frac{8}{9} \frac{D^{2}}{m_{0}}-\frac{16}{9} \frac{D F}{m_{0}}+\frac{8}{3} \frac{F^{2}}{m_{0}}+\frac{40}{9}\left(-b_{D}+b_{F}\right)+\frac{32}{3} b_{1}-\frac{32}{9}\left(b_{2}+b_{3}\right)-\frac{8}{3} m_{0} b_{5} \\
& +\frac{8}{9} m_{0}\left(b_{6}+b_{7}\right) \\
& \tilde{\epsilon}_{1, n}=\frac{1}{(4 \pi)^{2} F_{\pi}^{2}}\left(-\frac{25}{9} \frac{D^{2}}{m_{0}}+\frac{86}{9} \frac{D F}{m_{0}}+\frac{25}{3} \frac{F^{2}}{m_{0}}-\left(4+\frac{556}{9} D^{2}+\frac{136}{3} D F+60 F^{2}\right) b_{D}\right. \\
& -\left(4+\frac{68}{3} D^{2}+120 D F+60 F^{2}\right) b_{F}+4\left(b_{1}+b_{2}+b_{3}\right)+\frac{8}{3} m_{0} b_{5} \\
& \left.+\frac{8}{9} m_{0}\left(b_{6}-b_{7}\right)\right)+64\left(d_{1}+d_{2}+d_{3}+d_{5}\right) \text {, } \\
& \tilde{\epsilon}_{2, n}=\frac{1}{(4 \pi)^{2} F_{\pi}^{2}}\left(\frac{17}{9} \frac{D^{2}}{m_{0}}-\frac{70}{9} \frac{D F}{m_{0}}-\frac{17}{3} \frac{F^{2}}{m_{0}}+\left(4+\frac{68}{9} D^{2}+8 D F-12 F^{2}\right) b_{D}\right. \\
& +\left(4\left(1+D^{2}\right)-24 D F-12 F^{2}\right) b_{F}-4\left(b_{1}+b_{2}+b_{3}\right)-\frac{4}{3} m_{0} b_{5} \\
& \left.+\frac{4}{9} m_{0}\left(-b_{6}+b_{7}\right)\right)+64\left(d_{1}-d_{3}\right)-32 d_{5}, \\
& \tilde{\epsilon}_{3, n}=\frac{1}{(4 \pi)^{2} F_{\pi}^{2}}\left(\frac{8}{9} \frac{D^{2}}{m_{0}}-\frac{16}{9} \frac{D F}{m_{0}}-\frac{8}{3} \frac{F^{2}}{m_{0}}+\left(\frac{488}{9} D^{2}+\frac{112}{3} D F+72 F^{2}\right) b_{D}\right. \\
& \left.+\left(\frac{56}{3} D^{2}+144 D F+72 F^{2}\right) b_{F}-\frac{4}{3} m_{0} b_{5}+\frac{4}{9} m_{0}\left(-b_{6}+b_{7}\right)\right)-128 d_{1} \\
& -64 d_{2}-32 d_{5} \text {, } \\
& \tilde{\epsilon}_{4, n}=-2 \frac{D^{2}}{m_{0}}+4 \frac{D F}{m_{0}}+6 \frac{F^{2}}{m_{0}}-\left(16+48 D^{2}+96 D F+48 F^{2}\right)\left(b_{D}+b_{F}\right)+24 b_{1} \\
& +8\left(b_{2}-b_{3}\right)-6 m_{0} b_{5}+2 m_{0}\left(-b_{6}+b_{7}\right),
\end{aligned}
$$




$$
\begin{aligned}
& \tilde{\epsilon}_{5, n}=-\frac{2}{3} \frac{D^{2}}{m_{0}}+4 \frac{D F}{m_{0}}+2 \frac{F^{2}}{m_{0}}+\left(-8-\frac{104}{3} D^{2}+16 D F-24 F^{2}\right) b_{D} \\
& +\left(8\left(-1+D^{2}\right)-48 D F-24 F^{2}\right) b_{F}+8\left(b_{1}+b_{2}+b_{3}\right)-2 m_{0}\left(b_{5}+b_{6}+b_{7}+b_{9}\right) \text {, } \\
& \tilde{\epsilon}_{6, n}=\frac{2}{9} \frac{D^{2}}{m_{0}}-\frac{4}{9} \frac{D F}{m_{0}}-\frac{2}{3} \frac{F^{2}}{m_{0}}+\frac{16}{9}\left(b_{D}+b_{F}\right)-\frac{8}{3} b_{1}+\frac{8}{9}\left(-b_{2}+b_{3}\right)+\frac{2}{3} m_{0} b_{5} \\
& +\frac{2}{9} m_{0}\left(b_{6}-b_{7}\right) \\
& \tilde{\epsilon}_{7, n}=\frac{2}{3} \frac{D^{2}}{m_{0}}-4 \frac{D F}{m_{0}}-2 \frac{F^{2}}{m_{0}}+\left(8+\frac{64}{3} D^{2}\right) b_{D}+8\left(b_{F}-b_{1}-b_{2}-b_{3}\right) \\
& +2 m_{0}\left(b_{5}+b_{6}+b_{7}+b_{9}\right) \text {, } \\
& \tilde{\epsilon}_{8, n}=\frac{8}{9} \frac{D^{2}}{m_{0}}-\frac{16}{9} \frac{D F}{m_{0}}-\frac{8}{3} \frac{F^{2}}{m_{0}}+\frac{16}{9}\left(b_{D}+b_{F}\right)-\frac{32}{3} b_{1}+\frac{32}{9}\left(-b_{2}+b_{3}\right)+\frac{8}{3} m_{0} b_{5} \\
& +\frac{8}{9} m_{0}\left(b_{6}-b_{7}\right) \\
& \tilde{\epsilon}_{9, n}=\left(8+48 D^{2}+96 D F+48 F^{2}\right)\left(b_{D}+b_{F}\right) \text {, } \\
& \tilde{\epsilon}_{10, n}=\left(\frac{40}{3} D^{2}-16 D F+24 F^{2}\right) b_{D}+\left(-8 D^{2}+48 D F+24 F^{2}\right) b_{F}, \\
& \tilde{\epsilon}_{11, n}=\frac{8}{9} \frac{D^{2}}{m_{0}}-\frac{16}{9} \frac{D F}{m_{0}}-\frac{8}{3} \frac{F^{2}}{m_{0}}+\frac{40}{9}\left(b_{D}+b_{F}\right)-\frac{32}{3} b_{1}+\frac{32}{9}\left(-b_{2}+b_{3}\right)+\frac{8}{3} m_{0} b_{5} \\
& +\frac{8}{9} m_{0}\left(b_{6}-b_{7}\right) \\
& \tilde{\epsilon}_{1, \Xi^{0}}=\frac{1}{(4 \pi)^{2} F_{\pi}^{2}}\left(-\frac{25}{9} \frac{D^{2}}{m_{0}}-\frac{86}{9} \frac{D F}{m_{0}}+\frac{25}{3} \frac{F^{2}}{m_{0}}+\left(-4-\frac{556}{9} D^{2}+\frac{136}{3} D F-60 F^{2}\right) b_{D}\right. \\
& +\left(4+\frac{68}{3} D^{2}-120 D F+60 F^{2}\right) b_{F}+4\left(b_{1}-b_{2}+b_{3}\right)+\frac{8}{3} m_{0} b_{5} \\
& \left.-\frac{8}{9} m_{0}\left(b_{6}+b_{7}\right)\right)+64\left(d_{1}-d_{2}+d_{3}-d_{5}\right) \text {, } \\
& \tilde{\epsilon}_{2, \Xi^{0}}=\frac{1}{(4 \pi)^{2} F_{\pi}^{2}}\left(\frac{17}{9} \frac{D^{2}}{m_{0}}+\frac{70}{9} \frac{D F}{m_{0}}-\frac{17}{3} \frac{F^{2}}{m_{0}}+\left(4+\frac{68}{9} D^{2}-8 D F-12 F^{2}\right) b_{D}\right. \\
& +\left(-4\left(1+D^{2}\right)-24 D F+12 F^{2}\right) b_{F}+4\left(-b_{1}+b_{2}-b_{3}\right)-\frac{4}{3} m_{0} b_{5} \\
& \left.+\frac{4}{9} m_{0}\left(b_{6}+b_{7}\right)\right)+64\left(d_{1}-d_{3}\right)+32 d_{5}, \\
& \tilde{\epsilon}_{3, \Xi^{0}}=\frac{1}{(4 \pi)^{2} F_{\pi}^{2}}\left(\frac{8}{9} \frac{D^{2}}{m_{0}}+\frac{16}{9} \frac{D F}{m_{0}}-\frac{8}{3} \frac{F^{2}}{m_{0}}+\left(\frac{488}{9} D^{2}-\frac{112}{3} D F+72 F^{2}\right) b_{D}\right. \\
& \left.+\left(-\frac{56}{3} D^{2}+144 D F-72 F^{2}\right) b_{F}-\frac{4}{3} m_{0} b_{5}+\frac{4}{9} m_{0}\left(b_{6}+b_{7}\right)\right)-128 d_{1} \\
& +64 d_{2}+32 d_{5} \\
& \tilde{\epsilon}_{4, \Xi^{0}}=-2 \frac{D^{2}}{m_{0}}-4 \frac{D F}{m_{0}}+6 \frac{F^{2}}{m_{0}}+\left(-16-48 D^{2}+96 D F-48 F^{2}\right)\left(b_{D}-b_{F}\right)+24 b_{1} \\
& -8\left(b_{2}+b_{3}\right)-6 m_{0} b_{5}+2 m_{0}\left(b_{6}+b_{7}\right),
\end{aligned}
$$




$$
\begin{aligned}
\tilde{\epsilon}_{5, \Xi^{0}}= & -\frac{2}{3} \frac{D^{2}}{m_{0}}-4 \frac{D F}{m_{0}}+2 \frac{F^{2}}{m_{0}}-\left(8+\frac{104}{3} D^{2}+16 D F+24 F^{2}\right) b_{D} \\
+ & \left(8\left(1-D^{2}\right)-48 D F+24 F^{2}\right) b_{F}+8\left(b_{1}-b_{2}+b_{3}\right)+2 m_{0}\left(-b_{5}+b_{6}-b_{7}-b_{9}\right) \\
\tilde{\epsilon}_{6, \Xi^{0}}= & \frac{2}{9} \frac{D^{2}}{m_{0}}+\frac{4}{9} \frac{D F}{m_{0}}-\frac{2}{3} \frac{F^{2}}{m_{0}}+\frac{16}{9}\left(b_{D}-b_{F}\right)-\frac{8}{3} b_{1}+\frac{8}{9}\left(b_{2}+b_{3}\right)+\frac{2}{3} m_{0} b_{5} \\
& -\frac{2}{9} m_{0}\left(b_{6}+b_{7}\right) \\
\tilde{\epsilon}_{7, \Xi^{0}}= & \frac{2}{3} \frac{D^{2}}{m_{0}}+4 \frac{D F}{m_{0}}-2 \frac{F^{2}}{m_{0}}+\left(8+\frac{64}{3} D^{2}\right) b_{D}+8\left(-b_{F}-b_{1}+b_{2}-b_{3}\right) \\
+ & 2 m_{0}\left(b_{5}-b_{6}+b_{7}+b_{9}\right), \\
\tilde{\epsilon}_{8, \Xi^{0}} & =\frac{8}{9} \frac{D^{2}}{m_{0}}+\frac{16}{9} \frac{D F}{m_{0}}-\frac{8}{3} \frac{F^{2}}{m_{0}}+\frac{16}{9}\left(b_{D}-b_{F}\right)-\frac{32}{3} b_{1}+\frac{32}{9}\left(b_{2}+b_{3}\right)+\frac{8}{3} m_{0} b_{5} \\
& -\frac{8}{9} m_{0}\left(b_{6}+b_{7}\right), \\
\tilde{\epsilon}_{9, \Xi^{0}} & =\left(8+48 D^{2}-96 D F+48 F^{2}\right)\left(b_{D}-b_{F}\right), \\
\tilde{\epsilon}_{10, \Xi^{0}} & =\left(\frac{40}{3} D^{2}+16 D F+24 F^{2}\right) b_{D}+\left(8 D^{2}+48 D F-24 F^{2}\right) b_{F} \\
\tilde{\epsilon}_{11, \Xi^{0}} & =\frac{8}{9} \frac{D^{2}}{m_{0}}+\frac{16}{9} \frac{D F}{m_{0}}-\frac{8}{3} \frac{F^{2}}{m_{0}}+\frac{40}{9}\left(b_{D}-b_{F}\right)-\frac{32}{3} b_{1}+\frac{32}{9}\left(b_{2}+b_{3}\right)+\frac{8}{3} m_{0} b_{5} \\
& -\frac{8}{9} m_{0}\left(b_{6}+b_{7}\right) \cdot
\end{aligned}
$$

Note that from the dimension two (four) Lagrangian, the LECs $b_{4,8}\left(d_{4,6,7}\right)$ do not contribute to the strong isospin breaking corrections. There are no contributions $\sim b_{0}$ because this operator is only sensitive to the sum of the quark masses and also not affected by the $\Lambda-\Sigma^{0}$ mixing. While these expressions appear very voluminous and contain a fair amount of LECs, we show in the next paragraph how these can be constrained by reducing to the $\mathrm{SU}(2)$ case and some phenomenological results.

\section{Matching to the two-flavor case}

\subsection{Matching equations}

The quark mass expansions of the baryon masses given in the preceding section contain a sizeable amount of parameters (LECs). This does not pose a problem if one has sufficiently many lattice data at various quark masses. However, the fitting procedure to find the real minimum in this parameter space will be somewhat tedious and thus it is important to find further constraints on these parameters. This can be achieved for certain combinations of the LECs by matching to the $\mathrm{SU}(2)$ result for the nucleon mass. In the isospin limit $m_{u}=m_{d}$, we have $m_{p}=m_{n}=m_{N}$, and the quark mass expansion of $m_{N}$ is given by 
$[19,22,25]$

$$
\begin{aligned}
m_{N}=\tilde{m}_{0}-4 c_{1} \tilde{M}_{\pi}^{2}-\frac{3 g_{A}^{2}}{32 \pi F_{\pi}^{2}} \tilde{M}_{\pi}^{3}+\frac{3}{F_{\pi}^{2}}\left(2 c_{1}-\frac{c_{2}}{4}-c_{3}-\frac{g_{A}^{2}}{4 \tilde{m}_{0}}\right) \tilde{M}_{\pi}^{4} \bar{\mu} \\
+\left(-4 \bar{e}_{1}+\frac{3}{8} \frac{1}{\left(4 \pi F_{\pi}\right)^{2}} c_{2}-\frac{3}{4} \frac{g_{A}^{2}}{\left(4 \pi F_{\pi}\right)^{2}} \frac{1}{\tilde{m}_{0}}\right) \tilde{M}_{\pi}^{4}+\mathcal{O}\left(\tilde{M}_{\pi}^{5}\right) .
\end{aligned}
$$

Here, $\tilde{m}_{0}$ is the nucleon mass in the $\mathrm{SU}(2)$ chiral limit with $m_{u}=m_{d}=0$ and $m_{s}$ fixed at its physical value and $\tilde{M}_{\pi}$ is the $\mathrm{SU}(2)$ lowest order pion mass distinguished from its $\mathrm{SU}(3)$ counterpart $\bar{M}_{\pi}$ in Eqs. (2.10) by corrections in $m_{s}$. Strictly speaking, the same is true for the above $F_{\pi}$, yet, to the order we are working here, the difference is of no relevance. The $c_{i}$ are scale-independent LECs from the second order pion-nucleon Lagrangian [34] and $e_{1}$ is the sole scale-dependent combination of LECs that contributes to the nucleon mass $[19,31]$. In the notation of [31], we have $e_{1}=4 e_{38}+e_{115} / 2+e_{116} / 2$. Note that again we work at the scale given by the $\mathrm{SU}(2)$ nucleon mass in the chiral limit and thus use the LEC $\bar{e}_{1}$. These particular LECs are constrained from the various analyses of pion-nucleon scattering and pionic hydrogen/deuterium in chiral perturbation theory and the reaction $\pi N \rightarrow \pi \pi N$, see the next paragraph for a detailed discussion of this topic. Their numerical values can also be understood in terms of resonance saturation, see [35]; in particular they incorporate the important contribution from the $\Delta(1232)$ resonance. Mapping the quark mass expansion of $m_{N}$ onto Eq. (5.1), we obtain the following matching conditions:

\section{$\underline{\text { Chiral limit mass: }}$}

$$
\begin{aligned}
\tilde{m}_{0} & =\left(1+\left(-\frac{17}{12} b_{5}+\frac{17}{36} b_{6}-\frac{89}{108} b_{7}-\frac{13}{9} b_{8}-\frac{1}{4} b_{9}\right) \frac{\hat{M}_{K}^{4}}{\left(4 \pi F_{\pi}\right)^{2}}\right. \\
& +\left(\frac{3}{2} b_{5}-\frac{1}{2} b_{6}+\frac{3}{2} b_{7}+2 b_{8}+\frac{1}{2} b_{9}\right) \frac{\hat{M}_{K}^{4}}{\left(4 \pi F_{\pi}\right)^{2}} \ln \left(\frac{\hat{M}_{K}^{2}}{m_{0}^{2}}\right) \\
& \left.+\left(\frac{4}{3} b_{5}-\frac{4}{9} b_{6}+\frac{4}{27} b_{7}+\frac{8}{9} b_{8}\right) \frac{\hat{M}_{K}^{4}}{\left(4 \pi F_{\pi}\right)^{2}} \ln \left(\frac{4 \hat{M}_{K}^{2}}{3 m_{0}^{2}}\right)\right) m_{0} \\
& +4\left(-b_{0}-b_{D}+b_{F}\right) \hat{M}_{K}^{2}+\left(-\left(\frac{5}{12}+\frac{1}{9 \sqrt{3}}\right) D^{2}+\left(\frac{1}{2}+\frac{2}{3 \sqrt{3}}\right) D F\right. \\
& \left.-\left(\frac{3}{4}+\frac{1}{\sqrt{3}}\right) F^{2}\right) \frac{\hat{M}_{K}^{3}}{4 \pi F_{\pi}^{2}}+\left(\left(\frac{52}{9} D^{2}-\frac{40}{3} D F+4 F^{2}\right) \frac{b_{D}}{\left(4 \pi F_{\pi}\right)^{2}}\right. \\
& \left.+\left(-\frac{20}{3} D^{2}+8 D F-12 F^{2}\right) \frac{b_{F}}{\left(4 \pi F_{\pi}\right)^{2}}+16\left(-d_{1}+d_{2}-d_{3}+d_{5}-d_{6}-d_{7}\right)\right) \hat{M}_{K}^{4} \\
& +\left(8 b_{0}+\left(6+\frac{26}{3} D^{2}-20 D F+6 F^{2}\right) b_{D}+\left(-2-10 D^{2}+12 D F-18 F^{2}\right) b_{F}\right. \\
& \left.-6 b_{1}+2 b_{2}-6 b_{3}-8 b_{4}\right) \frac{\hat{M}_{K}^{4}}{\left(4 \pi F_{\pi}\right)^{2}} \ln \left(\frac{\hat{M}_{K}^{2}}{m_{0}^{2}}\right) \\
& +\left(\frac{32}{9}\left(b_{0}+b_{D}-b_{F}\right)-\frac{16}{3} b_{1}+\frac{16}{9} b_{2}-\frac{16}{27} b_{3}-\frac{32}{9} b_{4}\right) \frac{\hat{M}_{K}^{4}}{\left(4 \pi F_{\pi}\right)^{2}} \ln \left(\frac{4 \hat{M}_{K}^{2}}{3 m_{0}^{2}}\right)
\end{aligned}
$$




$$
\begin{aligned}
& +\left(\left(-\frac{53}{54} D^{2}+\frac{17}{9} D F-\frac{17}{6} F^{2}\right) \frac{\hat{M}_{K}^{4}}{\left(4 \pi F_{\pi}\right)^{2}}\right. \\
& +\left(-\frac{5}{6} D^{2}+D F-\frac{3}{2} F^{2}\right) \frac{\hat{M}_{K}^{4}}{\left(4 \pi F_{\pi}\right)^{2}} \ln \left(\frac{\hat{M}_{K}^{2}}{m_{0}^{2}}\right) \\
& \left.+\left(-\frac{4}{27} D^{2}+\frac{8}{9} D F-\frac{4}{3} F^{2}\right) \frac{\hat{M}_{K}^{4}}{\left(4 \pi F_{\pi}\right)^{2}} \ln \left(\frac{4 \hat{M}_{K}^{2}}{3 m_{0}^{2}}\right)\right) \frac{1}{m_{0}}+\mathcal{O}\left(\hat{M}_{K}^{5}\right)
\end{aligned}
$$

$\underline{\text { Axial-vector coupling: }}$

$$
g_{A}=D+F+\mathcal{O}\left(\hat{M}_{K}^{2}\right)
$$

Dimension two LECs:

$$
\begin{aligned}
c_{1} & =\left(\left(-\frac{3}{8} b_{5}+\frac{1}{8} b_{6}-\frac{3}{8} b_{7}-\frac{1}{2} b_{8}-\frac{1}{8} b_{9}\right) \frac{\hat{M}_{K}^{2}}{\left(4 \pi F_{\pi}\right)^{2}} \ln \left(\frac{\hat{M}_{K}^{2}}{m_{0}^{2}}\right)\right. \\
& \left.+\left(-\frac{1}{6} b_{5}+\frac{1}{18} b_{6}-\frac{1}{54} b_{7}-\frac{1}{9} b_{8}\right) \frac{\hat{M}_{K}^{2}}{\left(4 \pi F_{\pi}\right)^{2}} \ln \left(\frac{4 \hat{M}_{K}^{2}}{3 m_{0}^{2}}\right)\right) m_{0} \\
& +b_{0}+\frac{1}{2}\left(b_{D}+b_{F}\right)+\left(\left(\frac{5}{64}+\frac{1}{96 \sqrt{3}}\right) D^{2}-\left(\frac{3}{32}+\frac{1}{16 \sqrt{3}}\right) D F\right. \\
& \left.+\left(\frac{9}{64}+\frac{3}{32 \sqrt{3}}\right) F^{2}\right) \frac{\hat{M}_{K}}{4 \pi F_{\pi}^{2}}+\left(\left(16 L_{4}-32 L_{6}-\frac{11}{9} \frac{1}{(4 \pi)^{2}}\right) \frac{b_{0}}{F_{\pi}^{2}}\right. \\
& +\left(8 L_{4}-16 L_{6}+\left(-\frac{35}{36}-\frac{13}{12} D^{2}+\frac{5}{2} D F-\frac{3}{4} F^{2}\right) \frac{1}{(4 \pi)^{2}}\right) \frac{b_{D}}{F_{\pi}^{2}} \\
& +\left(8 L_{4}-16 L_{6}+\left(\frac{17}{36}+\frac{5}{4} D^{2}-\frac{3}{2} D F+\frac{9}{4} F^{2}\right) \frac{1}{(4 \pi)^{2}}\right) \frac{b_{F}}{F_{\pi}^{2}} \\
& \left.+\left(\frac{13}{12} b_{1}-\frac{13}{36} b_{2}+\frac{85}{108} b_{3}+\frac{11}{9} b_{4}\right) \frac{1}{\left(4 \pi F_{\pi}\right)^{2}}+4\left(-d_{1}+d_{3}\right)-2 d_{5}+8 d_{6}\right) \hat{M}_{K}^{2} \\
& +\left(-2 b_{0}-\frac{3}{2} b_{D}+\frac{1}{2} b_{F}+\frac{3}{2} b_{1}-\frac{1}{2} b_{2}+\frac{3}{2} b_{3}+2 b_{4}\right) \frac{\hat{M}_{K}^{2}}{\left(4 \pi F_{\pi}\right)^{2}} \ln \left(\frac{\hat{M}_{K}^{2}}{m_{0}^{2}}\right) \\
& \left.+\left(\frac{1}{54} D^{2}-\frac{1}{9} D F+\frac{1}{6} F^{2}\right) \frac{\hat{M}_{K}^{2}}{\left(4 \pi F_{\pi}\right)^{2}} \ln \left(\frac{4 \hat{M}_{K}^{2}}{3 m_{0}^{2}}\right)\right) \frac{1}{m_{0}}+\mathcal{O}\left(\hat{M}_{K}^{3}\right) \\
& +\left(\frac{2}{9}\left(-b_{0}-b_{D}+b_{F}\right)+\frac{2}{3} b_{1}-\frac{2}{9} b_{2}+\frac{2}{27} b_{3}+\frac{4}{9} b_{4}\right) \frac{\hat{M}_{K}^{2}}{\left(4 \pi F_{\pi}\right)^{2}} \ln \left(\frac{4 \hat{M}_{K}^{2}}{3 m_{0}^{2}}\right) \\
& +\left(\left(\frac{49}{144} D^{2}-\frac{13}{24} D F+\frac{13}{16} F^{2}\right) \frac{\hat{M}_{K}^{2}}{\left(4 \pi F_{\pi}\right)^{2}}\right. \\
& +\left(\frac{5}{24} D^{2}-\frac{1}{4} D F+\frac{3}{8} F^{2}\right) \frac{\hat{M}_{K}^{2}}{\left(4 \pi F_{\pi}\right)^{2}} \ln \left(\frac{\hat{M}_{K}^{2}}{m_{0}^{2}}\right) \\
& \left.+\left(b_{5}+b_{6}+b_{7}\right)+\frac{1}{2} b_{8}\right) m_{0}+b_{1}+b_{2}+b_{3}+2 b_{4}+\mathcal{O}\left(\hat{M}_{K}\right) \\
& +5
\end{aligned}
$$




\section{Dimension four LEC with recoil correction:}

$$
\begin{aligned}
& -4 \bar{e}_{1}+\frac{3}{8} \frac{1}{\left(4 \pi F_{\pi}\right)^{2}} c_{2}= \\
& \left(\left(\frac{1}{12} b_{5}-\frac{19}{36} b_{6}+\frac{1}{108} b_{7}-\frac{7}{36} b_{8}+\frac{1}{8} b_{9}\right) \frac{1}{\left(4 \pi F_{\pi}\right)^{2}}\right. \\
& +\left(\frac{3}{8} b_{5}-\frac{1}{8} b_{6}+\frac{3}{8} b_{7}+\frac{1}{2} b_{8}+\frac{1}{8} b_{9}\right) \frac{1}{\left(4 \pi F_{\pi}\right)^{2}} \ln \left(\frac{\hat{M}_{K}^{2}}{m_{0}^{2}}\right) \\
& \left.+\left(\frac{1}{12} b_{5}-\frac{1}{36} b_{6}+\frac{1}{108} b_{7}+\frac{1}{18} b_{8}\right) \frac{1}{\left(4 \pi F_{\pi}\right)^{2}} \ln \left(\frac{4 \hat{M}_{K}^{2}}{3 m_{0}^{2}}\right)\right) m_{0} \\
& +\left(-\left(\frac{5}{128}+\frac{1}{384 \sqrt{3}}\right) D^{2}+\left(\frac{3}{64}+\frac{1}{64 \sqrt{3}}\right) D F-\left(\frac{9}{128}+\frac{3}{128 \sqrt{3}}\right) F^{2}\right) \frac{1}{\hat{M}_{K} 4 \pi F_{\pi}^{2}} \\
& +\left(\frac{10}{3} b_{0}+\left(\frac{89}{36}-\frac{91}{36} D^{2}+\frac{35}{6} D F-\frac{7}{4} F^{2}\right) b_{D}+\left(-\frac{3}{4}+\frac{35}{12} D^{2}-\frac{7}{2} D F+\frac{21}{4} F^{2}\right) b_{F}\right. \\
& \left.-\frac{11}{4} b_{1}+\frac{11}{12} b_{2}-\frac{83}{36} b_{3}-\frac{10}{3} b_{4}\right) \frac{1}{\left(4 \pi F_{\pi}\right)^{2}}-4\left(d_{1}+d_{2}+d_{3}\right)-8 d_{5}-16 d_{6}-8 d_{7} \\
& +\left(2 b_{0}+\left(\frac{3}{2}-\frac{13}{6} D^{2}+5 D F-\frac{3}{2} F^{2}\right) b_{D}+\left(-\frac{1}{2}+\frac{5}{2} D^{2}-3 D F+\frac{9}{2} F^{2}\right) b_{F}\right. \\
& \left.-\frac{3}{2} b_{1}+\frac{1}{2} b_{2}-\frac{3}{2} b_{3}-2 b_{4}\right) \frac{1}{\left(4 \pi F_{\pi}\right)^{2}} \ln \left(\frac{\hat{M}_{K}^{2}}{m_{0}^{2}}\right) \\
& +\left(\frac{2}{9} b_{0}+\frac{1}{9}\left(b_{D}+b_{F}\right)-\frac{1}{3} b_{1}+\frac{1}{9} b_{2}-\frac{1}{27} b_{3}-\frac{2}{9} b_{4}\right) \frac{1}{\left(4 \pi F_{\pi}\right)^{2}} \ln \left(\frac{4 \hat{M}_{K}^{2}}{3 m_{0}^{2}}\right) \\
& +\left(\left(-\frac{235}{432} D^{2}+\frac{55}{72} D F-\frac{55}{48} F^{2}\right) \frac{1}{\left(4 \pi F_{\pi}\right)^{2}}\right. \\
& +\left(-\frac{5}{24} D^{2}+\frac{1}{4} D F-\frac{3}{8} F^{2}\right) \frac{1}{\left(4 \pi F_{\pi}\right)^{2}} \ln \left(\frac{\hat{M}_{K}^{2}}{m_{0}^{2}}\right) \\
& \left.+\left(-\frac{1}{108} D^{2}+\frac{1}{18} D F-\frac{1}{12} F^{2}\right) \frac{1}{\left(4 \pi F_{\pi}\right)^{2}} \ln \left(\frac{4 \hat{M}_{K}^{2}}{3 m_{0}^{2}}\right)\right) \frac{1}{m_{0}}+\mathcal{O}\left(\hat{M}_{K}\right),
\end{aligned}
$$

where $\hat{M}_{K}^{2}=m_{s} B$ is the leading strange quark contribution to the kaon mass. The first of these relations Eq. (5.2) connects the non-chiral part of the nucleon mass (generated by the gluon condensate) in the SU(2) limit with the one in the SU(3) case. Obviously, the only difference are terms proportional to powers of the strange quark mass (modulo logarithms). The difference between $m_{0}$ and $\tilde{m}_{0}$ is what is usually called the strangeness contribution to the nucleon mass. Eq. (5.3) is the usual reduction of the two axial-vector couplings in $\mathrm{SU}(3)$ to the single coupling $g_{A}$ for the two-flavor case. To the order we are working, this relation is unaffected by quark mass corrections. A similar statement holds for the relation between the dynamic dimension two LECs ${ }^{3}$ displayed in Eq. (5.5). Quite differently, the relation between the leading order symmetry breaking LEC in $\mathrm{SU}(2)$, called $c_{1}$, and its

\footnotetext{
${ }^{3}$ This name stems from the fact that they essentially contribute to pion/kaon-nucleon scattering.
} 
three $\mathrm{SU}(3)$ counterparts $b_{0, D, F}$, has sizeable quark mass corrections, simply because the corresponding operators contribute already to the baryon masses at first non-trivial order. In particular, one has corrections from the third order loop contributions that generate the terms $\sim \hat{M}_{K} \sim \sqrt{m_{s}}$. Finally, note that in the last relation the expansion of the loop functions $\bar{I}_{K, \eta}$ in powers of $M_{\pi}^{2}$ (using $\bar{M}_{K}^{2}=\hat{M}_{K}^{2}+\bar{M}_{\pi}^{2} / 2$ ) generates the contributions $\sim 1 / \hat{M}_{K} \sim 1 / \sqrt{m_{s}}$.

In the isospin breaking sector, we consider only the leading dimension two operator that

is parameterized by the LEC $c_{5}$ in $\mathrm{SU}(2)$ [35]. From matching the leading $\mathrm{SU}(3)$ terms $\sim\left(m_{u}-m_{d}\right)$ the following relation is obtained:

$$
\begin{aligned}
& c_{5}=\left(\left(\frac{1}{6} b_{5}+\frac{1}{18}\left(b_{6}-b_{7}\right)\right) \frac{\hat{M}_{K}^{2}}{\left(4 \pi F_{\pi}\right)^{2}}-\frac{1}{4}\left(b_{5}+b_{6}+b_{7}+b_{9}\right) \frac{\hat{M}_{K}^{2}}{\left(4 \pi F_{\pi}\right)^{2}} \ln \left(\frac{\hat{M}_{K}^{2}}{m_{0}^{2}}\right)\right. \\
& \left.+\left(-\frac{1}{3} b_{5}+\frac{1}{9}\left(-b_{6}+b_{7}\right)\right) \frac{\hat{M}_{K}^{2}}{\left(4 \pi F_{\pi}\right)^{2}} \ln \left(\frac{4 \hat{M}_{K}^{2}}{3 m_{0}^{2}}\right)\right) m_{0} \\
& +b_{D}+b_{F}+\left(-\left(\frac{1}{32}+\frac{1}{12 \sqrt{3}}\right) D^{2}+\left(\frac{3}{16}+\frac{1}{6 \sqrt{3}}\right) D F+\left(\frac{3}{32}+\frac{1}{4 \sqrt{3}}\right) F^{2}\right) \frac{\hat{M}_{K}}{4 \pi F_{\pi}^{2}} \\
& +\left(\left(16 L_{4}-32 L_{6}+\left(-\frac{1}{2}-\frac{17}{18} D^{2}-D F+\frac{3}{2} F^{2}\right) \frac{1}{(4 \pi)^{2}}\right) \frac{b_{D}}{F_{\pi}^{2}}\right. \\
& +\left(16 L_{4}-32 L_{6}+\left(-\frac{1}{2}\left(1+D^{2}\right)+3 D F+\frac{3}{2} F^{2}\right) \frac{1}{(4 \pi)^{2}}\right) \frac{b_{F}}{F_{\pi}^{2}} \\
& \left.+\frac{1}{2}\left(b_{1}+b_{2}+b_{3}\right) \frac{1}{\left(4 \pi F_{\pi}\right)^{2}}+8\left(-d_{1}+d_{3}\right)+4 d_{5}\right) \hat{M}_{K}^{2} \\
& +\left(-\left(1+\frac{8}{3} D^{2}\right) b_{D}-b_{F}+b_{1}+b_{2}+b_{3}\right) \frac{\hat{M}_{K}^{2}}{\left(4 \pi F_{\pi}\right)^{2}} \ln \left(\frac{\hat{M}_{K}^{2}}{m_{0}^{2}}\right) \\
& +\left(\frac{4}{3} b_{1}+\frac{4}{9}\left(b_{2}-b_{3}\right)\right) \frac{\hat{M}_{K}^{2}}{\left(4 \pi F_{\pi}\right)^{2}} \ln \left(\frac{4 \hat{M}_{K}^{2}}{3 m_{0}^{2}}\right) \\
& +\left(\left(-\frac{17}{72} D^{2}+\frac{35}{36} D F+\frac{17}{24} F^{2}\right) \frac{\hat{M}_{K}^{2}}{\left(4 \pi F_{\pi}\right)^{2}}\right. \\
& +\left(-\frac{1}{12} D^{2}+\frac{1}{2} D F+\frac{1}{4} F^{2}\right) \frac{\hat{M}_{K}^{2}}{\left(4 \pi F_{\pi}\right)^{2}} \ln \left(\frac{\hat{M}_{K}^{2}}{m_{0}^{2}}\right) \\
& \left.+\left(-\frac{1}{9} D^{2}+\frac{2}{9} D F+\frac{1}{3} F^{2}\right) \frac{\hat{M}_{K}^{2}}{\left(4 \pi F_{\pi}\right)^{2}} \ln \left(\frac{4 \hat{M}_{K}^{2}}{3 m_{0}^{2}}\right)\right) \frac{1}{m_{0}}+\mathcal{O}\left(\hat{M}_{K}^{3}\right) \text {. }
\end{aligned}
$$

\subsection{Bounds on the low-energy constants}

In this paragraph we collect what is known from various sources on the LECs appearing in the baryon masses. The $\mathrm{SU}(2)$ parameters have indeed been determined from the analysis

of pion-nucleon and nucleon-nucleon scattering. Combining the results from Refs. [35, 36, 37, 38], we find

$$
c_{1}=-0.9_{-0.2}^{+0.5}, \quad c_{2}=3.3 \pm 0.2, \quad c_{3}=-5.0_{-1.0}^{+1.6}, \quad c_{4}=3.5_{-0.2}^{+0.5},
$$


where all numbers are given in $\mathrm{GeV}^{-1}$. Furthermore, naturalness gives $-1 \lesssim e_{1} \lesssim 1 \mathrm{GeV}^{-3}$ (for $\lambda=1 \mathrm{GeV}$ ), which is consistent with the loose determination in [39]. Consequently, the left-hand-side (lhs) of Eq. (5.5) can be conservatively bounded by

$$
\frac{1}{4} c_{2}+c_{3}=-5.2 \ldots-2.5 \mathrm{GeV}^{-1}
$$

and similarly for the lhs of Eq. (5.6)

$$
3 \mathrm{GeV}^{-3} \leq-4 \bar{e}_{1}+\frac{3}{8} \frac{1}{\left(4 \pi F_{\pi}\right)^{2}} c_{2} \leq 5 \mathrm{GeV}^{-3} .
$$

These constraints are also consistent with recent lattice interpolations of $\mathrm{SU}(2)$ results, one has for Eqs. $(5.9,5.10)-3.1(-3.9 \ldots-2.6)$ and $5(3.2 \ldots 5.7)$ in $[5]([6])$, respectively. We now turn to estimating the SU(3) LECs $b_{i}$ and $d_{i}$. The symmetry breaking LECs $b_{0, D, F}$ can be determined from a third order fit to the baryon masses. We collect here the results from the comprehensive study in [17],

$$
-0.79 \leq b_{0} \leq-0.70, \quad 0.01 \leq b_{D} \leq 0.07, \quad-0.61 \leq b_{F} \leq-0.48,
$$

with all numbers given in $\mathrm{GeV}^{-1}$. The uncertainties on these numbers are certainly underestimated by looking only at the baryon masses, see the discussion in [18]. The resonance saturation estimates presented in that paper also give somewhat different values, but it should be noted that this method is not expected to work well for the LECs related to symmetry breaking. For orientation, one can use Eq. (68) of [18] for getting a rough idea of the size of the dimension four LECs $d_{i}$, provided one performs the relabeling $d_{7}^{\mathrm{BM}} \rightarrow d_{6}$ and $d_{8}^{\mathrm{BM}} \rightarrow d_{7}$. For the dynamical dimension two LECs $b_{i}(i=1, \ldots, 9)$, resonance saturation should work better. We map here the results of [18] onto our notation so that one can get some estimates about the size of some combinations of the dynamical dimension two LECs (we refrain here from redoing the calculations of that paper since these numbers only serve as a rough guide). We find the following relations

$$
\begin{aligned}
& b_{1}^{\mathrm{BM}} \hat{=} b_{1}-\frac{m_{0}}{4} b_{5}-\frac{m_{0}}{16} b_{9}, \\
& b_{2}^{\mathrm{BM}} \hat{=} b_{2}-\frac{m_{0}}{4} b_{6} \\
& b_{3}^{\mathrm{BM}} \hat{=} b_{3}-\frac{m_{0}}{4} b_{7}-\frac{3 m_{0}}{16} b_{9} \\
& b_{8}^{\mathrm{BM}} \hat{=} b_{4}-\frac{m_{0}}{4} b_{8}+\frac{m_{0}}{8} b_{9}
\end{aligned}
$$

and the pertinent resonance saturation estimates for the $b_{i}^{\mathrm{BM}}$ can be found in Eq. (68) of [18]. Last, we consider the leading isospin breaking LEC $c_{5}$. It was determined in [35] $c_{5}=-0.09 \pm 0.01 \mathrm{GeV}^{-1}$. Note that in [40] a somewhat larger value was obtained based on a leading order $\mathrm{SU}(3)$ estimate for the strong proton-neutron mass difference.

\section{Sigma terms}

Directly related to the baryon masses are the so-called sigma terms that measure the contribution of the QCD quark mass term to any baryon mass,

$$
\sigma_{q B}(0)=\left\langle B\left|m_{q} \bar{q} q\right| B\right\rangle .
$$


Here $q$ denotes any of the light quark flavors $\mathrm{u}$, $\mathrm{d}$ or s. These scalar-isoscalar operators can be directly read off from the quark mass expansion of the baryon masses given in the preceding section. We refrain from displaying the lengthy formulas here. Instead, we briefly discuss the so-called pion-nucleon sigma term. It measures the strength of the light quark condensate in the proton (and can be determined from the analytically continued isoscalar pion-nucleon scattering amplitude $\left.\bar{D}^{+}(\nu, t)\right)$,

$$
\sigma_{\pi N}(0)=\hat{m}\langle p|\bar{u} u+\bar{d} d| p\rangle=\hat{m} \frac{\partial m_{N}}{\partial \hat{m}},
$$

where the last equality follows from the Feynman-Hellmann theorem [41]. More precisely, what is given in Eq. (6.2) is the pion-nucleon sigma term in the isospin limit, its generalization to include isospin violation is obtained by the substitution $\hat{m}(\bar{u} u+\bar{d} d) \rightarrow m_{u} \bar{u} u+m_{d} \bar{d} d$. We will only consider the isospin symmetric case here and refer the reader to Refs.[42, 43] for a discussion of the effects generated by strong and electromagnetic isospin violation. In terms of the so-called strangeness fraction $y$, the sigma term can be expressed as

$$
\sigma_{\pi N}(0)=\frac{\hat{\sigma}}{1-y}
$$

with

$$
\begin{aligned}
\hat{\sigma} & =\hat{m}\langle p|\bar{u} u+\bar{d} d-2 \bar{s} s| p\rangle, \\
y & =\frac{2\langle p|\bar{s} s| p\rangle}{\langle p|\bar{u} u+\bar{d} d| p\rangle} .
\end{aligned}
$$

These two quantities can also be read off from the quark mass expansion of the nucleon mass via

$$
\begin{aligned}
& \hat{\sigma}=\hat{m} \frac{\partial m_{N}}{\partial \hat{m}}-2 \hat{m} \frac{\partial m_{N}}{\partial m_{s}}, \\
& y=2 \frac{\partial m_{N}}{\partial m_{s}}\left(\frac{\partial m_{N}}{\partial \hat{m}}\right)^{-1},
\end{aligned}
$$

which allows for a direct determination from the lattice result for $m_{N}=m_{N}\left(m_{u}, m_{d}, m_{s}\right)$. Chiral perturbation theory calculations to order $m_{q}^{2}$ give $\hat{\sigma}=35 \pm 5 \mathrm{MeV}[15], \hat{\sigma}=36 \pm$ $7 \mathrm{MeV}$ [18], and $\hat{\sigma}=33 \pm 3 \mathrm{MeV}$ [23]. For a recent analysis of the sigma term in view of SU(2) lattice results, see [6]. For earlier lattice studies of this interesting quantity, see [44]-[48]. Similarly, one can also analyze the two kaon-nucleon sigma terms, $\sigma_{K N}^{(1)}(0)=$ $\left(\hat{m}+m_{s}\right)\langle p|\bar{u} u+\bar{s} s| p\rangle / 2$ and $\sigma_{K N}^{(2)}(0)=\left(\hat{m}+m_{s}\right)\langle p|-\bar{u} u+2 \bar{d} d+\bar{s} s| p\rangle / 2$, in the isospin limit. The strange quark contribution to the nucleon mass can be directly expressed as a linear combination of the pion-nucleon and the two kaon-nucleon sigma terms via

$$
m_{s}\langle p|\bar{s} s| p\rangle=\left(\frac{1}{2}-\frac{\bar{M}_{\pi}^{2}}{4 \bar{M}_{K}^{2}}\right)\left[3 \sigma_{K N}^{(1)}(0)+\sigma_{K N}^{(2)}(0)\right]+\left(\frac{1}{2}-\frac{\bar{M}_{K}^{2}}{\bar{M}_{\pi}^{2}}\right) \sigma_{\pi N}(0) .
$$

Similar analyses can be performed for the other baryons, but only in the case of the nucleon sigma terms one can hope to compare lattice results to the ones of phenomenological investigations. 


\section{Summary and conclusions}

We summarize the main results of our work:

1) We have calculated the octet baryon masses to fourth order in the chiral expansion within a Lorentz-invariant formulation of baryon chiral perturbation theory. In contrast to earlier works (with the exception of Ref. [15] which utilizes an UV cut-off) we have systematically included strong isospin breaking, $m_{u} \neq m_{d}$. This amounts to considering all terms quadratic in the quark masses.

2) To disentangle the dependence of the baryon masses on the three light quark masses, one has to consider the dimension two and four Lagrangians displayed in Eq. (2.14) and Eq. (2.16), respectively. At dimension two, one has three symmetry breaking LECs $\left(b_{0}, b_{D}, b_{F}\right)$ and nine dynamical LECs, that enter at fourth order in the tadpole diagrams. At dimension four, we have seven LECs, which appear in various combinations in the masses.

3) We have derived chiral extrapolation functions for the octet ground state baryons, including all isospin breaking terms linear in the mixing angle $\varepsilon$, see Section 4 . These constitute the main result of this paper and might be used to analyze unquenched three-flavor simulations at varying quark masses above their physical values. The equations collected in this section can be obtained as a Mathematica notebook from the authors upon request. The corresponding meson mass representations of the baryon masses that are not truncated at order $\varepsilon$ are given in App. A.

4) We have performed the matching to the two-flavor case to obtain constraints on various combinations of dimension two and four SU(3) low-energy constants, see Section 5. We have also reviewed the determination of LECs in SU(2) and the symmetry breaking dimension two SU(3) LECs and also given resonance saturation estimates for the SU(3) LECs, based on the earlier work in [18].

5) The various sigma terms as defined in Eq. (6.1) can be obtained by differentiation of the mass formulas with respect to the quark masses. The corresponding extrapolation functions can be obtained from the authors upon request.

\section{Acknowledgements}

We thank Bastian Kubis for some useful comments in the initial stages of this investigation. 


\section{A. Meson mass representation}

Here, we give the explicit representations of the baryon masses in terms of the Goldstone boson masses. The local terms generated by the dimension two and four insertions from the effective Lagrangian are expressed in terms of the charged pion and kaon masses throughout. The loop contributions at third and fourth order are expressed in terms of the loop functions $\bar{I}_{P}, \bar{\mu}_{P}$, and $\bar{I}_{P}^{12}$, compare Eq. (3.7), Eq. (3.6), and Eq. (3.9), respectively. Here, $P$ stands for any of the charged and neutral pseudoscalar mesons. To arrive at the quark mass expansion given in Section4, one has to expand these loop functions as given there and further expand the various mixing functions to linear order in $\varepsilon$. Note that only the lowest order meson mass relations are used to convert to the quark masses, that means

in the quark mass representation we employ the chiral value of $B$ throughout (while the meson mass representation is formulated in physical masses to the order we are working here).

The various contributions to the baryon masses can be compactly written as

$$
\begin{aligned}
m_{B}^{(2)} & =\gamma_{B}^{\pi} M_{\pi^{+}}^{2}+\gamma_{B}^{K} M_{K^{+}}^{2}, \\
m_{B}^{(3)} & =\delta_{1, B} \bar{I}_{\pi^{+}}+\delta_{2, B} \bar{I}_{\pi^{0}}+\delta_{3, B} \bar{I}_{K^{+}}+\delta_{4, B} \bar{I}_{K^{0}}+\delta_{5, B} \bar{I}_{\eta}, \\
m_{B}^{(4)} & =\epsilon_{1, B} \bar{\mu}_{\pi^{+}}+\epsilon_{2, B} \bar{\mu}_{\pi^{0}}+\epsilon_{3, B} \bar{\mu}_{K^{+}}+\epsilon_{4, B} \bar{\mu}_{K^{0}}+\epsilon_{5, B} \bar{\mu}_{\eta} \\
& +\epsilon_{6, B} \bar{I}_{\pi^{+}}^{12}+\epsilon_{7, B} \bar{I}_{\pi^{0}}^{12}+\epsilon_{8, B} \bar{I}_{K^{+}}^{12}+\epsilon_{9, B} \bar{I}_{K^{0}}^{12}+\epsilon_{10, B} \bar{I}_{\eta}^{12} \\
& +\epsilon_{11, B}^{\pi} M_{\pi^{+}}^{4}+\epsilon_{11, B}^{K} M_{K^{+}}^{4}+\epsilon_{11, B}^{\pi K} M_{\pi^{+}}^{2} M_{K^{+}}^{2}
\end{aligned}
$$

with

$$
\begin{aligned}
\delta_{i, B} & =\frac{m_{0}}{F_{\pi}^{2}}\left(\delta_{i, B}^{\pi} M_{\pi^{+}}^{2}+\delta_{i, B}^{K} M_{K^{+}}^{2}\right), \quad(i=1, \ldots, 5), \\
\epsilon_{i, B} & =\frac{1}{F_{\pi}^{2}}\left(\epsilon_{i, B}^{\pi} M_{\pi^{+}}^{4}+\epsilon_{i, B}^{K} M_{K^{+}}^{4}+\epsilon_{i, B}^{\pi K} M_{\pi^{+}}^{2} M_{K^{+}}^{2}\right), \quad(i=1, \ldots, 5), \\
\epsilon_{j, B} & =\frac{m_{0}^{2}}{F_{\pi}^{2}}\left(\epsilon_{j, B}^{\pi} M_{\pi^{+}}^{4}+\epsilon_{j, B}^{K} M_{K^{+}}^{4}+\epsilon_{j, B}^{\pi K} M_{\pi^{+}}^{2} M_{K^{+}}^{2}\right), \quad(j=6, \ldots, 10) .
\end{aligned}
$$

For the $\Lambda$ and $\Sigma^{0}$ one has additional fourth order terms due to the mixing which take the form

$$
\begin{aligned}
& \delta m_{\Sigma^{0}}^{(4)}=\frac{1}{\eta_{7}\left(M_{\pi^{+}}^{2}-M_{K^{+}}^{2}\right)} \sum_{i=1}^{6}\left(\eta_{i}^{\pi} M_{\pi^{+}}^{4}+\eta_{i}^{K} M_{K^{+}}^{4}+\eta_{i}^{\pi K} M_{\pi^{+}}^{2} M_{K^{+}}^{2}\right) \frac{m_{0}^{2}}{F_{\pi}^{4}} \bar{A}_{i}, \\
& \delta m_{\Lambda}^{(4)}=-\delta m_{\Sigma^{0}}^{(4)}
\end{aligned}
$$

with $\bar{A}_{1}=\bar{I}_{\pi^{+}}^{2}, \bar{A}_{2}=\bar{I}_{\pi^{+}} \bar{I}_{K^{+}}, \bar{A}_{3}=\bar{I}_{\pi^{+}} \bar{I}_{K^{0}}, \bar{A}_{4}=\bar{I}_{K^{+}}^{2}, \bar{A}_{5}=\bar{I}_{K^{+}} \bar{I}_{K^{0}}$, and $\bar{A}_{6}=\bar{I}_{K^{0}}^{2}$.

In what follows, we only display the non-vanishing coefficients $\gamma_{B}, \delta_{B}$, and $\epsilon_{B}$. 
The second order coefficients $\gamma_{B}$ are:

$$
\begin{aligned}
\gamma_{\Sigma^{+}}^{\pi} & =(-6+4 I) b_{0}-4 b_{D}+8(1-I) b_{F} \\
\gamma_{\Sigma^{+}}^{K} & =-4 I b_{0}+8(-1+I) b_{F} \\
\gamma_{\Sigma^{-}}^{\pi} & =(-6+4 I) b_{0}-4 b_{D}+8(-1+I) b_{F} \\
\gamma_{\Sigma^{-}}^{K} & =-4 I b_{0}+8(1-I) b_{F}, \\
\gamma_{\Sigma^{0}}^{\pi} & =(-6+4 I) b_{0}+\left(-4+\frac{8}{3} I-\frac{8}{3} \cos (2 \varepsilon)+\frac{1}{\sqrt{3}}\left(8-\frac{32}{3} I\right) \sin (2 \varepsilon)\right) b_{D}, \\
\gamma_{\Sigma^{0}}^{K} & =-4 I b_{0}+\left(-\frac{8}{3} I+\frac{8}{3} \cos (2 \varepsilon)+\frac{1}{\sqrt{3}}\left(-8+\frac{32}{3} I\right) \sin (2 \varepsilon)\right) b_{D}, \\
\gamma_{p}^{\pi} & =(-6+4 I) b_{0}+(4-8 I) b_{F}, \\
\gamma_{p}^{K} & =-4 I b_{0}-4 b_{D}+(-4+8 I) b_{F}, \\
\gamma_{\Xi^{-}}^{\pi} & =(-6+4 I) b_{0}+(-4+8 I) b_{F}, \\
\gamma_{\Xi^{-}}^{K} & =-4 I b_{0}-4 b_{D}+(4-8 I) b_{F}, \\
\gamma_{n}^{\pi} & =(-6+4 I) b_{0}+8(-1+I) b_{D}-4 b_{F}, \\
\gamma_{n}^{K} & =-4 I b_{0}+(4-8 I) b_{D}+4 b_{F}, \\
\gamma_{\Xi^{0}}^{\pi} & =(-6+4 I) b_{0}+8(-1+I) b_{D}+4 b_{F}, \\
\gamma_{\Xi^{0}}^{K} & =-4 I b_{0}+(4-8 I) b_{D}-4 b_{F}, \\
\gamma_{\Lambda}^{\pi} & =(-6+4 I) b_{0}+\left(-4+\frac{8}{3} I+\frac{8}{3} \cos (2 \varepsilon)+\frac{1}{\sqrt{3}}\left(-8+\frac{32}{3} I\right) \sin (2 \varepsilon)\right) b_{D}, \\
\gamma_{\Lambda}^{K} & =-4 I b_{0}+\left(-\frac{8}{3} I-\frac{8}{3} \cos (2 \varepsilon)+\frac{1}{\sqrt{3}}\left(8-\frac{32}{3} I\right) \sin (2 \varepsilon)\right) b_{D},
\end{aligned}
$$

with

$$
I=\frac{\sqrt{3}}{\sqrt{3}-\tan (2 \varepsilon)} \simeq 1+\frac{2 \varepsilon}{\sqrt{3}},
$$

and furthermore $I=1$ in the isospin limit $m_{u}=m_{d}$.

The third order coefficients $\delta_{B}$ are:

$$
\begin{array}{ll}
\delta_{1, \Sigma^{+}}^{\pi}=\frac{2}{3} D^{2}+2 F^{2}, & \delta_{1, \Sigma^{-}}^{\pi}=\frac{2}{3} D^{2}+2 F^{2}, \\
\delta_{1, \Sigma^{0}}^{\pi}=\frac{2}{3}(1-\cos (2 \varepsilon)) D^{2}+2(1+\cos (2 \varepsilon)) F^{2}, & \delta_{1, p}^{\pi}=D^{2}+2 D F+F^{2}, \\
\delta_{1, \Xi^{-}}^{\pi}=D^{2}-2 D F+F^{2}, \\
\delta_{1, n}^{\pi}=D^{2}+2 D F+F^{2}, \\
\delta_{1, \Xi^{0}}^{\pi}=D^{2}-2 D F+F^{2} \\
\delta_{1, \Lambda}^{\pi}=\frac{2}{3}(1+\cos (2 \varepsilon)) D^{2}+2(1-\cos (2 \varepsilon)) F^{2},
\end{array}
$$




$$
\begin{aligned}
& \delta_{2, \Sigma^{+}}^{\pi}=\left(\frac{1}{3}-\frac{4}{9} I+\frac{1}{9} \cos (2 \varepsilon)+\frac{1}{\sqrt{3}}\left(-\frac{2}{3}+\frac{10}{9} I\right) \sin (2 \varepsilon)\right) D^{2}+\left(-\frac{4}{3}+\frac{4}{3} I\right. \\
& \left.+\frac{1}{\sqrt{3}}\left(2-\frac{4}{3} I\right) \sin (2 \varepsilon)\right) D F+\left(1+\cos (2 \varepsilon)+\frac{2}{\sqrt{3}}(-1+I) \sin (2 \varepsilon)\right) F^{2}, \\
& \delta_{2, \Sigma^{+}}^{K}=\left(\frac{4}{9} I-\frac{4}{9} \cos (2 \varepsilon)+\frac{1}{\sqrt{3}}\left(\frac{2}{3}-\frac{10}{9} I\right) \sin (2 \varepsilon)\right) D^{2}+\frac{4}{3}\left(1-I+\frac{\sin (2 \varepsilon)}{\sqrt{3}} I\right) D F \\
& +\frac{2}{\sqrt{3}}(1-I) \sin (2 \varepsilon) F^{2}, \\
& \delta_{2, \Sigma^{-}}^{\pi}=\left(\frac{1}{3}-\frac{4}{9} I+\frac{1}{9} \cos (2 \varepsilon)+\frac{1}{\sqrt{3}}\left(-\frac{2}{3}+\frac{10}{9} I\right) \sin (2 \varepsilon)\right) D^{2}+\left(\frac{4}{3}-\frac{4}{3} I\right. \\
& \left.+\frac{1}{\sqrt{3}}\left(-2+\frac{4}{3} I\right) \sin (2 \varepsilon)\right) D F+\left(1+\cos (2 \varepsilon)+\frac{2}{\sqrt{3}}(-1+I) \sin (2 \varepsilon)\right) F^{2}, \\
& \delta_{2, \Sigma^{-}}^{K}=\left(\frac{4}{9} I-\frac{4}{9} \cos (2 \varepsilon)+\frac{1}{\sqrt{3}}\left(\frac{2}{3}-\frac{10}{9} I\right) \sin (2 \varepsilon)\right) D^{2}+\frac{4}{3}\left(-1+I-\frac{\sin (2 \varepsilon)}{\sqrt{3}} I\right) D F \\
& +\frac{2}{\sqrt{3}}(1-I) \sin (2 \varepsilon) F^{2} \text {, } \\
& \delta_{2, \Sigma^{0}}^{\pi}=\left(\frac{2}{3}-\frac{4}{9} I+\frac{4}{9} \cos (2 \varepsilon)+\frac{1}{\sqrt{3}}\left(-\frac{4}{3}+\frac{16}{9} I\right) \sin (2 \varepsilon)\right) D^{2}, \\
& \delta_{2, \Sigma^{0}}^{K}=\left(\frac{4}{9} I-\frac{4}{9} \cos (2 \varepsilon)+\frac{1}{\sqrt{3}}\left(\frac{4}{3}-\frac{16}{9} I\right) \sin (2 \varepsilon)\right) D^{2}, \\
& \delta_{2, p}^{\pi}=\left(\frac{2}{3}-\frac{4}{9} I+\frac{5}{18} \cos (2 \varepsilon)+\frac{1}{\sqrt{3}}\left(-\frac{7}{6}+\frac{10}{9} I\right) \sin (2 \varepsilon)\right) D^{2}+\left(-\frac{2}{3}+\frac{4}{3} I+\frac{1}{3} \cos (2 \varepsilon)\right. \\
& \left.+\frac{1}{\sqrt{3}}\left(1-\frac{4}{3} I\right) \sin (2 \varepsilon)\right) D F+\left(\frac{1}{2} \cos (2 \varepsilon)+\frac{1}{\sqrt{3}}\left(-\frac{1}{2}+2 I\right) \sin (2 \varepsilon)\right) F^{2}, \\
& \delta_{2, p}^{K}=\left(-\frac{1}{3}+\frac{4}{9} I-\frac{1}{9} \cos (2 \varepsilon)+\frac{1}{\sqrt{3}}\left(\frac{2}{3}-\frac{10}{9} I\right) \sin (2 \varepsilon)\right) D^{2}+\left(\frac{2}{3}-\frac{4}{3} I+\frac{2}{3} \cos (2 \varepsilon)\right. \\
& \left.+\frac{4}{3} \frac{\sin (2 \varepsilon)}{\sqrt{3}} I\right) D F+\left(1-\cos (2 \varepsilon)+\frac{2}{\sqrt{3}}(1-I) \sin (2 \varepsilon)\right) F^{2} \\
& \delta_{2, \Xi^{-}}^{\pi}=\left(\frac{2}{3}-\frac{4}{9} I+\frac{5}{18} \cos (2 \varepsilon)+\frac{1}{\sqrt{3}}\left(-\frac{7}{6}+\frac{10}{9} I\right) \sin (2 \varepsilon)\right) D^{2}+\left(\frac{2}{3}-\frac{4}{3} I-\frac{1}{3} \cos (2 \varepsilon)\right. \\
& \left.+\frac{1}{\sqrt{3}}\left(-1+\frac{4}{3} I\right) \sin (2 \varepsilon)\right) D F+\left(\frac{1}{2} \cos (2 \varepsilon)+\frac{1}{\sqrt{3}}\left(-\frac{1}{2}+2 I\right) \sin (2 \varepsilon)\right) F^{2}, \\
& \delta_{2, \Xi^{-}}^{K}=\left(-\frac{1}{3}+\frac{4}{9} I-\frac{1}{9} \cos (2 \varepsilon)+\frac{1}{\sqrt{3}}\left(\frac{2}{3}-\frac{10}{9} I\right) \sin (2 \varepsilon)\right) D^{2}+\left(-\frac{2}{3}+\frac{4}{3} I-\frac{2}{3} \cos (2 \varepsilon)\right. \\
& \left.-\frac{4}{3} \frac{\sin (2 \varepsilon)}{\sqrt{3}} I\right) D F+\left(1-\cos (2 \varepsilon)+\frac{2}{\sqrt{3}}(1-I) \sin (2 \varepsilon)\right) F^{2},
\end{aligned}
$$




$$
\begin{aligned}
& \delta_{2, n}^{\pi}=\left(\frac{2}{9} I+\frac{5}{18} \cos (2 \varepsilon)+\frac{1}{\sqrt{3}}\left(-\frac{1}{6}+\frac{4}{9} I\right) \sin (2 \varepsilon)\right) D^{2}+\left(\frac{2}{3}+\frac{1}{3} \cos (2 \varepsilon)-\frac{\sin (2 \varepsilon)}{\sqrt{3}}\right) D F \\
& +\left(2-2 I+\frac{1}{2} \cos (2 \varepsilon)+\frac{1}{\sqrt{3}}\left(-\frac{7}{2}+4 I\right) \sin (2 \varepsilon)\right) F^{2}, \\
& \delta_{2, n}^{K}=\left(\frac{1}{3}-\frac{2}{9} I-\frac{1}{9} \cos (2 \varepsilon)+\frac{1}{\sqrt{3}}\left(\frac{2}{3}-\frac{4}{9} I\right) \sin (2 \varepsilon)\right) D^{2}+\frac{2}{3}(-1+\cos (2 \varepsilon)) D F \\
& +\left(-1+2 I-\cos (2 \varepsilon)+\frac{1}{\sqrt{3}}(2-4 I) \sin (2 \varepsilon)\right) F^{2} \\
& \delta_{2, \Xi^{0}}^{\pi}=\left(\frac{2}{9} I+\frac{5}{18} \cos (2 \varepsilon)+\frac{1}{\sqrt{3}}\left(-\frac{1}{6}+\frac{4}{9} I\right) \sin (2 \varepsilon)\right) D^{2}+\left(-\frac{2}{3}-\frac{1}{3} \cos (2 \varepsilon)+\frac{\sin (2 \varepsilon)}{\sqrt{3}}\right) D F \\
& +\left(2-2 I+\frac{1}{2} \cos (2 \varepsilon)+\frac{1}{\sqrt{3}}\left(-\frac{7}{2}+4 I\right) \sin (2 \varepsilon)\right) F^{2}, \\
& \delta_{2, \Xi^{0}}^{K}=\left(\frac{1}{3}-\frac{2}{9} I-\frac{1}{9} \cos (2 \varepsilon)+\frac{1}{\sqrt{3}}\left(\frac{2}{3}-\frac{4}{9} I\right) \sin (2 \varepsilon)\right) D^{2}+\frac{2}{3}(1-\cos (2 \varepsilon)) D F \\
& +\left(-1+2 I-\cos (2 \varepsilon)+\frac{1}{\sqrt{3}}(2-4 I) \sin (2 \varepsilon)\right) F^{2}, \\
& \delta_{2, \Lambda}^{\pi}=\left(\frac{2}{3}-\frac{4}{9} I+\frac{4}{9} \cos (2 \varepsilon)+\frac{1}{\sqrt{3}}\left(-\frac{4}{3}+\frac{16}{9} I\right) \sin (2 \varepsilon)\right) D^{2}, \\
& \delta_{2, \Lambda}^{K}=\left(\frac{4}{9} I-\frac{4}{9} \cos (2 \varepsilon)+\frac{1}{\sqrt{3}}\left(\frac{4}{3}-\frac{16}{9} I\right) \sin (2 \varepsilon)\right) D^{2}, \\
& \delta_{3, \Sigma^{+}}^{K}=D^{2}+2 D F+F^{2}, \quad \delta_{3, \Sigma^{-}}^{K}=D^{2}-2 D F+F^{2}, \\
& \delta_{3, \Sigma^{0}}^{K}=\left(\frac{2}{3}+\frac{1}{3} \cos (2 \varepsilon)-\frac{\sin (2 \varepsilon)}{\sqrt{3}}\right) D^{2}+(2-\cos (2 \varepsilon)+\sqrt{3} \sin (2 \varepsilon)) F^{2} \\
& \delta_{3, p}^{K}=\frac{2}{3} D^{2}+2 F^{2}, \quad \delta_{3, \Xi^{-}}^{K}=\frac{2}{3} D^{2}+2 F^{2}, \\
& \delta_{3, n}^{K}=D^{2}-2 D F+F^{2}, \quad \delta_{3, \Xi^{0}}^{K}=D^{2}+2 D F+F^{2}, \\
& \delta_{3, \Lambda}^{K}=\left(\frac{2}{3}-\frac{1}{3} \cos (2 \varepsilon)+\frac{\sin (2 \varepsilon)}{\sqrt{3}}\right) D^{2}+(2+\cos (2 \varepsilon)-\sqrt{3} \sin (2 \varepsilon)) F^{2}, \\
& \delta_{4, \Sigma^{+}}^{\pi}=2(1-I)\left(D^{2}-2 D F+F^{2}\right), \quad \delta_{4, \Sigma^{+}}^{K}=(-1+2 I)\left(D^{2}-2 D F+F^{2}\right), \\
& \delta_{4, \Sigma^{-}}^{\pi}=2(1-I)\left(D^{2}+2 D F+F^{2}\right), \quad \delta_{4, \Sigma^{-}}^{K}=(-1+2 I)\left(D^{2}+2 D F+F^{2}\right) \text {, } \\
& \delta_{4, \Sigma^{0}}^{\pi}=\left(\frac{4}{3}-\frac{4}{3} I+\frac{1}{\sqrt{3}}\left(2-\frac{8}{3} I\right) \sin (2 \varepsilon)\right) D^{2}+\left(4-4 I+\frac{1}{\sqrt{3}}(-6+8 I) \sin (2 \varepsilon)\right) F^{2}, \\
& \delta_{4, \Sigma^{0}}^{K}=\left(-\frac{2}{3}+\frac{4}{3} I+\frac{1}{3} \cos (2 \varepsilon)+\frac{1}{\sqrt{3}}\left(-1+\frac{8}{3} I\right) \sin (2 \varepsilon)\right) D^{2} \\
& +\left(-2+4 I-\cos (2 \varepsilon)+\frac{1}{\sqrt{3}}(3-8 I) \sin (2 \varepsilon)\right) F^{2},
\end{aligned}
$$




$$
\begin{aligned}
& \delta_{4, p}^{\pi}=2(1-I)\left(D^{2}-2 D F+F^{2}\right), \quad \delta_{4, p}^{K}=(-1+2 I)\left(D^{2}-2 D F+F^{2}\right), \\
& \delta_{4, \Xi^{-}}^{\pi}=2(1-I)\left(D^{2}+2 D F+F^{2}\right), \quad \delta_{4, \Xi^{-}}^{K}=(-1+2 I)\left(D^{2}+2 D F+F^{2}\right) \text {, } \\
& \delta_{4, n}^{\pi}=\frac{4}{3}(1-I) D^{2}+4(1-I) F^{2}, \quad \delta_{4, n}^{K}=\left(-\frac{2}{3}+\frac{4}{3} I\right) D^{2}+(-2+4 I) F^{2}, \\
& \delta_{4, \Xi^{0}}^{\pi}=\frac{4}{3}(1-I) D^{2}+4(1-I) F^{2}, \quad \delta_{4, \Xi^{0}}^{K}=\left(-\frac{2}{3}+\frac{4}{3} I\right) D^{2}+(-2+4 I) F^{2}, \\
& \delta_{4, \Lambda}^{\pi}=\left(\frac{4}{3}-\frac{4}{3} I+\frac{1}{\sqrt{3}}\left(-2+\frac{8}{3} I\right) \sin (2 \varepsilon)\right) D^{2}+\left(4-4 I+\frac{1}{\sqrt{3}}(6-8 I) \sin (2 \varepsilon)\right) F^{2}, \\
& \delta_{4, \Lambda}^{K}=\left(-\frac{2}{3}+\frac{4}{3} I-\frac{1}{3} \cos (2 \varepsilon)+\frac{1}{\sqrt{3}}\left(1-\frac{8}{3} I\right) \sin (2 \varepsilon)\right) D^{2} \\
& +\left(-2+4 I+\cos (2 \varepsilon)+\frac{1}{\sqrt{3}}(-3+8 I) \sin (2 \varepsilon)\right) F^{2}, \\
& \delta_{5, \Sigma^{+}}^{\pi}=\left(\frac{1}{3}-\frac{4}{9} I-\frac{1}{9} \cos (2 \varepsilon)+\frac{1}{\sqrt{3}}\left(\frac{2}{3}-\frac{10}{9} I\right) \sin (2 \varepsilon)\right) D^{2}+\left(-\frac{4}{3}+\frac{4}{3} I\right. \\
& \left.+\frac{1}{\sqrt{3}}\left(-2+\frac{4}{3} I\right) \sin (2 \varepsilon)\right) D F+\left(1-\cos (2 \varepsilon)+\frac{2}{\sqrt{3}}(1-I) \sin (2 \varepsilon)\right) F^{2}, \\
& \delta_{5, \Sigma^{+}}^{K}=\left(\frac{4}{9} I+\frac{4}{9} \cos (2 \varepsilon)+\frac{1}{\sqrt{3}}\left(-\frac{2}{3}+\frac{10}{9} I\right) \sin (2 \varepsilon)\right) D^{2}+\frac{4}{3}\left(1-I-\frac{\sin (2 \varepsilon)}{\sqrt{3}} I\right) D F \\
& +\frac{2}{\sqrt{3}}(-1+I) \sin (2 \varepsilon) F^{2} \text {, } \\
& \delta_{5, \Sigma^{-}}^{\pi}=\left(\frac{1}{3}-\frac{4}{9} I-\frac{1}{9} \cos (2 \varepsilon)+\frac{1}{\sqrt{3}}\left(\frac{2}{3}-\frac{10}{9} I\right) \sin (2 \varepsilon)\right) D^{2}+\left(\frac{4}{3}-\frac{4}{3} I\right. \\
& \left.+\frac{1}{\sqrt{3}}\left(2-\frac{4}{3} I\right) \sin (2 \varepsilon)\right) D F+\left(1-\cos (2 \varepsilon)+\frac{2}{\sqrt{3}}(1-I) \sin (2 \varepsilon)\right) F^{2}, \\
& \delta_{5, \Sigma^{-}}^{K}=\left(\frac{4}{9} I+\frac{4}{9} \cos (2 \varepsilon)+\frac{1}{\sqrt{3}}\left(-\frac{2}{3}+\frac{10}{9} I\right) \sin (2 \varepsilon)\right) D^{2}+\frac{4}{3}\left(-1+I+\frac{\sin (2 \varepsilon)}{\sqrt{3}} I\right) D F \\
& +\frac{2}{\sqrt{3}}(-1+I) \sin (2 \varepsilon) F^{2} \\
& \delta_{5, \Sigma^{0}}^{\pi}=\left(\frac{2}{3}-\frac{4}{9} I-\frac{4}{9} \cos (2 \varepsilon)+\frac{1}{\sqrt{3}}\left(\frac{4}{3}-\frac{16}{9} I\right) \sin (2 \varepsilon)\right) D^{2}, \\
& \delta_{5, \Sigma^{0}}^{K}=\left(\frac{4}{9} I+\frac{4}{9} \cos (2 \varepsilon)+\frac{1}{\sqrt{3}}\left(-\frac{4}{3}+\frac{16}{9} I\right) \sin (2 \varepsilon)\right) D^{2}, \\
& \delta_{5, p}^{\pi}=\left(\frac{2}{3}-\frac{4}{9} I-\frac{5}{18} \cos (2 \varepsilon)+\frac{1}{\sqrt{3}}\left(\frac{7}{6}-\frac{10}{9} I\right) \sin (2 \varepsilon)\right) D^{2}+\left(-\frac{2}{3}+\frac{4}{3} I-\frac{1}{3} \cos (2 \varepsilon)\right. \\
& \left.+\frac{1}{\sqrt{3}}\left(-1+\frac{4}{3} I\right) \sin (2 \varepsilon)\right) D F+\left(-\frac{1}{2} \cos (2 \varepsilon)+\frac{1}{\sqrt{3}}\left(\frac{1}{2}-2 I\right) \sin (2 \varepsilon)\right) F^{2}, \\
& \delta_{5, p}^{K}=\left(-\frac{1}{3}+\frac{4}{9} I+\frac{1}{9} \cos (2 \varepsilon)+\frac{1}{\sqrt{3}}\left(-\frac{2}{3}+\frac{10}{9} I\right) \sin (2 \varepsilon)\right) D^{2}+\left(\frac{2}{3}-\frac{4}{3} I-\frac{2}{3} \cos (2 \varepsilon)\right. \\
& \left.-\frac{4}{3} \frac{\sin (2 \varepsilon)}{\sqrt{3}} I\right) D F+\left(1+\cos (2 \varepsilon)+\frac{2}{\sqrt{3}}(-1+I) \sin (2 \varepsilon)\right) F^{2},
\end{aligned}
$$




$$
\begin{aligned}
& \delta_{5, \Xi^{-}}^{\pi}=\left(\frac{2}{3}-\frac{4}{9} I-\frac{5}{18} \cos (2 \varepsilon)+\frac{1}{\sqrt{3}}\left(\frac{7}{6}-\frac{10}{9} I\right) \sin (2 \varepsilon)\right) D^{2}+\left(\frac{2}{3}-\frac{4}{3} I+\frac{1}{3} \cos (2 \varepsilon)\right. \\
& \left.+\frac{1}{\sqrt{3}}\left(1-\frac{4}{3} I\right) \sin (2 \varepsilon)\right) D F+\left(-\frac{1}{2} \cos (2 \varepsilon)+\frac{1}{\sqrt{3}}\left(\frac{1}{2}-2 I\right) \sin (2 \varepsilon)\right) F^{2}, \\
& \delta_{5, \Xi^{-}}^{K}=\left(-\frac{1}{3}+\frac{4}{9} I+\frac{1}{9} \cos (2 \varepsilon)+\frac{1}{\sqrt{3}}\left(-\frac{2}{3}+\frac{10}{9} I\right) \sin (2 \varepsilon)\right) D^{2}+\left(-\frac{2}{3}+\frac{4}{3} I+\frac{2}{3} \cos (2 \varepsilon)\right. \\
& \left.+\frac{4}{3} \frac{\sin (2 \varepsilon)}{\sqrt{3}} I\right) D F+\left(1+\cos (2 \varepsilon)+\frac{2}{\sqrt{3}}(-1+I) \sin (2 \varepsilon)\right) F^{2}, \\
& \delta_{5, n}^{\pi}=\left(\frac{2}{9} I-\frac{5}{18} \cos (2 \varepsilon)+\frac{1}{\sqrt{3}}\left(\frac{1}{6}-\frac{4}{9} I\right) \sin (2 \varepsilon)\right) D^{2}+\left(\frac{2}{3}-\frac{1}{3} \cos (2 \varepsilon)+\frac{\sin (2 \varepsilon)}{\sqrt{3}}\right) D F \\
& +\left(2-2 I-\frac{1}{2} \cos (2 \varepsilon)+\frac{1}{\sqrt{3}}\left(\frac{7}{2}-4 I\right) \sin (2 \varepsilon)\right) F^{2}, \\
& \delta_{5, n}^{K}=\left(\frac{1}{3}-\frac{2}{9} I+\frac{1}{9} \cos (2 \varepsilon)+\frac{1}{\sqrt{3}}\left(-\frac{2}{3}+\frac{4}{9} I\right) \sin (2 \varepsilon)\right) D^{2}-\frac{2}{3}(1+\cos (2 \varepsilon)) D F \\
& +\left(-1+2 I+\cos (2 \varepsilon)+\frac{1}{\sqrt{3}}(-2+4 I) \sin (2 \varepsilon)\right) F^{2}, \\
& \delta_{5, \Xi^{0}}^{\pi}=\left(\frac{2}{9} I-\frac{5}{18} \cos (2 \varepsilon)+\frac{1}{\sqrt{3}}\left(\frac{1}{6}-\frac{4}{9} I\right) \sin (2 \varepsilon)\right) D^{2}+\left(-\frac{2}{3}+\frac{1}{3} \cos (2 \varepsilon)-\frac{\sin (2 \varepsilon)}{\sqrt{3}}\right) D F \\
& +\left(2-2 I-\frac{1}{2} \cos (2 \varepsilon)+\frac{1}{\sqrt{3}}\left(\frac{7}{2}-4 I\right) \sin (2 \varepsilon)\right) F^{2}, \\
& \delta_{5, \Xi^{0}}^{K}=\left(\frac{1}{3}-\frac{2}{9} I+\frac{1}{9} \cos (2 \varepsilon)+\frac{1}{\sqrt{3}}\left(-\frac{2}{3}+\frac{4}{9} I\right) \sin (2 \varepsilon)\right) D^{2}+\frac{2}{3}(1+\cos (2 \varepsilon)) D F \\
& +\left(-1+2 I+\cos (2 \varepsilon)+\frac{1}{\sqrt{3}}(-2+4 I) \sin (2 \varepsilon)\right) F^{2}, \\
& \delta_{5, \Lambda}^{\pi}=\left(\frac{2}{3}-\frac{4}{9} I-\frac{4}{9} \cos (2 \varepsilon)+\frac{1}{\sqrt{3}}\left(\frac{4}{3}-\frac{16}{9} I\right) \sin (2 \varepsilon)\right) D^{2}, \\
& \delta_{5, \Lambda}^{K}=\left(\frac{4}{9} I+\frac{4}{9} \cos (2 \varepsilon)+\frac{1}{\sqrt{3}}\left(-\frac{4}{3}+\frac{16}{9} I\right) \sin (2 \varepsilon)\right) D^{2} .
\end{aligned}
$$

Finally, the fourth order coefficients $\epsilon_{B}$ are (since these formulas are very long, we only give here the expressions for the proton and the neutron. The others, including the coefficients $\eta_{i}, i=1, \ldots 7$, in Eq. (A.7) of the non-diagonal contributions to the $\Sigma^{0}$ and $\Lambda$ masses, can be obtained from the authors upon request):

$$
\begin{aligned}
\epsilon_{1, p}^{\pi} & =4 b_{0}+\left(2-4 D^{2}-8 D F-4 F^{2}\right)\left(b_{D}+b_{F}\right)-2\left(b_{1}+b_{2}+b_{3}\right)-4 b_{4}+\frac{m_{0}}{2}\left(b_{5}+b_{6}+b_{7}\right) \\
& +m_{0} b_{8}+\left(4 D^{2}+8 D F+4 F^{2}\right)\left(b_{D}+b_{F}\right) I, \\
\epsilon_{1, p}^{\pi K} & =\left(4 D^{2}+8 D F+4 F^{2}\right)\left(b_{D}+b_{F}\right)(1-I), \\
\epsilon_{1, n}^{\pi} & =4 b_{0}+\left(2+4 D^{2}+8 D F+4 F^{2}\right)\left(b_{D}+b_{F}\right)-2\left(b_{1}+b_{2}+b_{3}\right)-4 b_{4}+\frac{m_{0}}{2}\left(b_{5}+b_{6}+b_{7}\right) \\
& +m_{0} b_{8}-\left(4 D^{2}+8 D F+4 F^{2}\right)\left(b_{D}+b_{F}\right) I, \\
\epsilon_{1, n}^{\pi K} & =\left(4 D^{2}+8 D F+4 F^{2}\right)\left(b_{D}+b_{F}\right)(-1+I),
\end{aligned}
$$




$$
\begin{aligned}
& \epsilon_{2, p}^{\pi}=\frac{17}{3} b_{0}+\frac{2}{3} b_{D}-\frac{4}{3} b_{F}-\frac{2}{3} b_{1}+\frac{4}{3} b_{2}-\frac{26}{9} b_{3}-\frac{14}{3} b_{4}+\frac{m_{0}}{6} b_{5}-\frac{m_{0}}{3} b_{6}+\frac{13}{18} m_{0} b_{7}+\frac{7}{6} m_{0} b_{8} \\
& +\left(4 b_{0}+\frac{8}{3}\left(b_{D}-b_{1}\right)+\frac{16}{9} b_{2}-\frac{56}{27} b_{3}-\frac{40}{9} b_{4}+\frac{2}{3} m_{0} b_{5}-\frac{4}{9} m_{0} b_{6}+\frac{14}{27} m_{0} b_{7}+\frac{10}{9} m_{0} b_{8}\right) I^{2} \\
& +\left(-8 b_{0}+\frac{8}{3}\left(-b_{D}+b_{F}+b_{1}\right)-\frac{32}{9} b_{2}+\frac{40}{9} b_{3}+8 b_{4}-\frac{2}{3} m_{0} b_{5}+\frac{8}{9} m_{0} b_{6}-\frac{10}{9} m_{0} b_{7}\right. \\
& \left.-2 m_{0} b_{8}\right) I+\left(\frac{4}{3} b_{0}+\frac{1}{3} b_{D}+\frac{5}{3} b_{F}-\frac{1}{3} b_{1}-\frac{5}{9} b_{2}-\frac{13}{27} b_{3}-\frac{8}{9} b_{4}+\frac{m_{0}}{12} b_{5}+\frac{5}{36} m_{0} b_{6}\right. \\
& \left.+\frac{13}{108} m_{0} b_{7}+\frac{2}{9} m_{0} b_{8}\right) \cos (2 \varepsilon)+\frac{1}{\sqrt{3}}\left(-10 b_{0}-b_{D}+3 b_{F}+b_{1}-\frac{7}{3} b_{2}+5 b_{3}+8 b_{4}\right. \\
& -\frac{m_{0}}{4} b_{5}+\frac{7}{12} m_{0} b_{6}-\frac{5}{4} m_{0} b_{7}-2 m_{0} b_{8}+\left(-8 b_{0}-\frac{8}{3} b_{D}+8 b_{F}+\frac{8}{3} b_{1}-\frac{40}{9} b_{2}+\frac{104}{27} b_{3}\right. \\
& \left.+\frac{64}{9} b_{4}-\frac{2}{3} m_{0} b_{5}+\frac{10}{9} m_{0} b_{6}-\frac{26}{27} m_{0} b_{7}-\frac{16}{9} m_{0} b_{8}\right) I^{2}+\left(16 b_{0}+4 b_{D}-\frac{20}{3} b_{F}-4 b_{1}\right. \\
& \left.\left.+\frac{52}{9} b_{2}-\frac{220}{27} b_{3}-\frac{128}{9} b_{4}+m_{0} b_{5}-\frac{13}{9} m_{0} b_{6}+\frac{55}{27} m_{0} b_{7}+\frac{32}{9} m_{0} b_{8}\right) I\right) \sin (2 \varepsilon), \\
& \epsilon_{2, p}^{K}=\frac{8}{3}\left(b_{0}+b_{D}+b_{F}-b_{1}\right)-\frac{8}{9} b_{3}-\frac{8}{3} b_{4}+\frac{2}{3} m_{0} b_{5}+\frac{2}{9} m_{0} b_{7}+\frac{2}{3} m_{0} b_{8}+\left(4 b_{0}\right. \\
& \left.+\frac{8}{3}\left(b_{D}-b_{1}\right)+\frac{16}{9} b_{2}-\frac{56}{27} b_{3}-\frac{40}{9} b_{4}+\frac{2}{3} m_{0} b_{5}-\frac{4}{9} m_{0} b_{6}+\frac{14}{27} m_{0} b_{7}+\frac{10}{9} m_{0} b_{8}\right) I^{2} \\
& +\left(-4 b_{0}-\frac{8}{3} b_{D}-\frac{16}{3} b_{F}+\frac{8}{3} b_{1}-\frac{8}{9} b_{2}+\frac{8}{3} b_{3}+\frac{16}{3} b_{4}-\frac{2}{3} m_{0} b_{5}+\frac{2}{9} m_{0} b_{6}-\frac{2}{3} m_{0} b_{7}\right. \\
& \left.-\frac{4}{3} m_{0} b_{8}\right) I+\left(\frac{8}{3}\left(-b_{0}-b_{D}+b_{F}+b_{1}\right)-\frac{8}{9} b_{2}+\frac{8}{27} b_{3}+\frac{16}{9} b_{4}-\frac{2}{3} m_{0} b_{5}+\frac{2}{9} m_{0} b_{6}\right. \\
& \left.-\frac{2}{27} m_{0} b_{7}-\frac{4}{9} m_{0} b_{8}\right) \cos (2 \varepsilon)+\frac{1}{\sqrt{3}}\left(4\left(b_{D}+b_{F}-b_{1}\right)+\frac{4}{3}\left(-b_{2}+b_{3}\right)+m_{0} b_{5}\right. \\
& +\frac{m_{0}}{3}\left(b_{6}-b_{7}\right)+\left(-8 b_{0}-\frac{8}{3} b_{D}+8 b_{F}+\frac{8}{3} b_{1}-\frac{40}{9} b_{2}+\frac{104}{27} b_{3}+\frac{64}{9} b_{4}-\frac{2}{3} m_{0} b_{5}\right. \\
& \left.+\frac{10}{9} m_{0} b_{6}-\frac{26}{27} m_{0} b_{7}-\frac{16}{9} m_{0} b_{8}\right) I^{2}+\left(4\left(b_{0}-b_{D}\right)-\frac{20}{3} b_{F}+4 b_{1}+\frac{28}{9} b_{2}-\frac{100}{27} b_{3}\right. \\
& \left.\left.-\frac{32}{9} b_{4}-m_{0} b_{5}-\frac{7}{9} m_{0} b_{6}+\frac{25}{27} m_{0} b_{7}+\frac{8}{9} m_{0} b_{8}\right) I\right) \sin (2 \varepsilon)
\end{aligned}
$$




$$
\begin{aligned}
& \epsilon_{2, p}^{\pi K}=-\frac{16}{3} b_{0}+\frac{4}{3}\left(-b_{D}-b_{F}+b_{1}-b_{2}\right)+\frac{28}{9} b_{3}+\frac{16}{3} b_{4}+\frac{m_{0}}{3}\left(-b_{5}+b_{6}\right)-\frac{7}{9} m_{0} b_{7} \\
& -\frac{4}{3} m_{0} b_{8}+\left(-8 b_{0}+\frac{16}{3}\left(-b_{D}+b_{1}\right)-\frac{32}{9} b_{2}+\frac{112}{27} b_{3}+\frac{80}{9} b_{4}-\frac{4}{3} m_{0} b_{5}+\frac{8}{9} m_{0} b_{6}\right. \\
& \left.-\frac{28}{27} m_{0} b_{7}-\frac{20}{9} m_{0} b_{8}\right) I^{2}+\left(12 b_{0}+\frac{16}{3} b_{D}+\frac{8}{3} b_{F}-\frac{16}{3} b_{1}+\frac{40}{9} b_{2}-\frac{64}{9} b_{3}-\frac{40}{3} b_{4}\right. \\
& \left.+\frac{4}{3} m_{0} b_{5}-\frac{10}{9} m_{0} b_{6}+\frac{16}{9} m_{0} b_{7}+\frac{10}{3} m_{0} b_{8}\right) I+\left(\frac{4}{3}\left(b_{0}+b_{D}-b_{F}-b_{1}\right)+\frac{4}{9} b_{2}\right. \\
& \left.-\frac{4}{27} b_{3}-\frac{8}{9} b_{4}+\frac{m_{0}}{3} b_{5}-\frac{m_{0}}{9} b_{6}+\frac{m_{0}}{27} b_{7}+\frac{2}{9} m_{0} b_{8}\right) \cos (2 \varepsilon) \\
& +\frac{1}{\sqrt{3}}\left(10 b_{0}-4 b_{F}+\frac{8}{3} b_{2}-\frac{16}{3} b_{3}-8 b_{4}-\frac{2}{3} m_{0} b_{6}+\frac{4}{3} m_{0} b_{7}+2 m_{0} b_{8}\right. \\
& +\left(16 b_{0}+\frac{16}{3} b_{D}-16 b_{F}-\frac{16}{3} b_{1}+\frac{80}{9} b_{2}-\frac{208}{27} b_{3}-\frac{128}{9} b_{4}+\frac{4}{3} m_{0} b_{5}-\frac{20}{9} m_{0} b_{6}\right. \\
& \left.+\frac{52}{27} m_{0} b_{7}+\frac{32}{9} m_{0} b_{8}\right) I^{2}+\left(-20 b_{0}+\frac{40}{3} b_{F}-\frac{80}{9} b_{2}+\frac{320}{27} b_{3}+\frac{160}{9} b_{4}\right. \\
& \left.\left.+\frac{20}{9} m_{0} b_{6}-\frac{80}{27} m_{0} b_{7}-\frac{40}{9} m_{0} b_{8}\right) I\right) \sin (2 \varepsilon) \text {, } \\
& \epsilon_{2, n}^{\pi}=\frac{17}{3} b_{0}+6 b_{D}+4 b_{F}-\frac{26}{3} b_{1}-\frac{4}{3} b_{2}-\frac{2}{9} b_{3}-\frac{14}{3} b_{4}+\frac{13}{6} m_{0} b_{5}+\frac{m_{0}}{3} b_{6}+\frac{m_{0}}{18} b_{7} \\
& +\frac{7}{6} m_{0} b_{8}+\left(4 b_{0}+\frac{8}{3} b_{D}-8 b_{1}-\frac{8}{27} b_{3}-\frac{40}{9} b_{4}+2 m_{0} b_{5}+\frac{2}{27} m_{0} b_{7}+\frac{10}{9} m_{0} b_{8}\right) I^{2} \\
& +\left(-8\left(b_{0}+b_{D}\right)-\frac{8}{3} b_{F}+16 b_{1}+\frac{8}{9} b_{2}+8 b_{4}-4 m_{0} b_{5}-\frac{2}{9} m_{0} b_{6}-2 m_{0} b_{8}\right) I \\
& +\left(\frac{4}{3} b_{0}+\frac{1}{3} b_{D}+\frac{5}{3} b_{F}-\frac{1}{3} b_{1}-\frac{5}{9} b_{2}-\frac{13}{27} b_{3}-\frac{8}{9} b_{4}+\frac{m_{0}}{12} b_{5}+\frac{5}{36} m_{0} b_{6}+\frac{13}{108} m_{0} b_{7}\right. \\
& \left.+\frac{2}{9} m_{0} b_{8}\right) \cos (2 \varepsilon)+\frac{1}{\sqrt{3}}\left(-10 b_{0}-11 b_{D}-7 b_{F}+15 b_{1}+\frac{7}{3} b_{2}+\frac{1}{3} b_{3}+8 b_{4}\right. \\
& -\frac{15}{4} m_{0} b_{5}-\frac{7}{12} m_{0} b_{6}-\frac{m_{0}}{12} b_{7}-2 m_{0} b_{8}+\left(-8 b_{0}-\frac{32}{3} b_{D}+16 b_{1}-\frac{16}{27} b_{3}\right. \\
& \left.+\frac{64}{9} b_{4}-4 m_{0} b_{5}+\frac{4}{27} m_{0} b_{7}-\frac{16}{9} m_{0} b_{8}\right) I^{2}+\left(16 b_{0}+\frac{52}{3} b_{D}+\frac{20}{3} b_{F}-28 b_{1}\right. \\
& \left.\left.-\frac{20}{9} b_{2}-\frac{4}{27} b_{3}-\frac{128}{9} b_{4}+7 m_{0} b_{5}+\frac{5}{9} m_{0} b_{6}+\frac{m_{0}}{27} b_{7}+\frac{32}{9} m_{0} b_{8}\right) I\right) \sin (2 \varepsilon) \text {, }
\end{aligned}
$$




$$
\begin{aligned}
& \epsilon_{2, n}^{K}=\frac{8}{3}\left(b_{0}-b_{1}\right)-\frac{8}{9} b_{3}-\frac{8}{3} b_{4}+\frac{2}{3} m_{0} b_{5}+\frac{2}{9} m_{0} b_{7}+\frac{2}{3} m_{0} b_{8} \\
& +\left(4 b_{0}+\frac{8}{3} b_{D}-8 b_{1}-\frac{8}{27} b_{3}-\frac{40}{9} b_{4}+2 m_{0} b_{5}+\frac{2}{27} m_{0} b_{7}+\frac{10}{9} m_{0} b_{8}\right) I^{2} \\
& +\left(-4 b_{0}-\frac{8}{3} b_{F}+8 b_{1}+\frac{8}{9}\left(b_{2}+b_{3}\right)+\frac{16}{3} b_{4}-2 m_{0} b_{5}-\frac{2}{9} m_{0}\left(b_{6}+b_{7}\right)-\frac{4}{3} m_{0} b_{8}\right) I \\
& +\left(\frac{8}{3}\left(-b_{0}-b_{D}+b_{F}+b_{1}\right)-\frac{8}{9} b_{2}+\frac{8}{27} b_{3}+\frac{16}{9} b_{4}-\frac{2}{3} m_{0} b_{5}+\frac{2}{9} m_{0} b_{6}-\frac{2}{27} m_{0} b_{7}\right. \\
& \left.-\frac{4}{9} m_{0} b_{8}\right) \cos (2 \varepsilon)+\frac{1}{\sqrt{3}}\left(4\left(-b_{D}-b_{F}+b_{1}\right)+\frac{4}{3}\left(b_{2}-b_{3}\right)-m_{0} b_{5}+\frac{m_{0}}{3}\left(-b_{6}+b_{7}\right)\right. \\
& +\left(-8 b_{0}-\frac{32}{3} b_{D}+16 b_{1}-\frac{16}{27} b_{3}+\frac{64}{9} b_{4}-4 m_{0} b_{5}+\frac{4}{27} m_{0} b_{7}-\frac{16}{9} m_{0} b_{8}\right) I^{2} \\
& +\left(4 b_{0}+\frac{28}{3} b_{D}+\frac{20}{3} b_{F}-12 b_{1}-\frac{20}{9} b_{2}+\frac{44}{27} b_{3}-\frac{32}{9} b_{4}+3 m_{0} b_{5}+\frac{5}{9} m_{0} b_{6}\right. \\
& \left.\left.-\frac{11}{27} m_{0} b_{7}+\frac{8}{9} m_{0} b_{8}\right) I\right) \sin (2 \varepsilon) \text {, } \\
& \epsilon_{2, n}^{\pi K}=-\frac{16}{3} b_{0}-4\left(b_{D}+b_{F}\right)+\frac{28}{3} b_{1}+\frac{4}{3} b_{2}+\frac{4}{9} b_{3}+\frac{16}{3} b_{4}-\frac{7}{3} m_{0} b_{5}-\frac{m_{0}}{3} b_{6}-\frac{m_{0}}{9} b_{7} \\
& -\frac{4}{3} m_{0} b_{8}+\left(-8 b_{0}-\frac{16}{3} b_{D}+16 b_{1}+\frac{16}{27} b_{3}+\frac{80}{9} b_{4}-4 m_{0} b_{5}-\frac{4}{27} m_{0} b_{7}-\frac{20}{9} m_{0} b_{8}\right) I^{2} \\
& +\left(12 b_{0}+8 b_{D}+\frac{16}{3} b_{F}-24 b_{1}-\frac{16}{9} b_{2}-\frac{8}{9} b_{3}-\frac{40}{3} b_{4}+6 m_{0} b_{5}+\frac{4}{9} m_{0} b_{6}+\frac{2}{9} m_{0} b_{7}\right. \\
& \left.+\frac{10}{3} m_{0} b_{8}\right) I+\left(\frac{4}{3}\left(b_{0}+b_{D}-b_{F}-b_{1}\right)+\frac{4}{9} b_{2}-\frac{4}{27} b_{3}-\frac{8}{9} b_{4}+\frac{m_{0}}{3} b_{5}-\frac{m_{0}}{9} b_{6}\right. \\
& \left.+\frac{m_{0}}{27} b_{7}+\frac{2}{9} m_{0} b_{8}\right) \cos (2 \varepsilon)+\frac{1}{\sqrt{3}}\left(10 b_{0}+12 b_{D}+8 b_{F}-16 b_{1}-\frac{8}{3} b_{2}-8 b_{4}\right. \\
& +4 m_{0} b_{5}+\frac{2}{3} m_{0} b_{6}+2 m_{0} b_{8}+\left(16 b_{0}+\frac{64}{3} b_{D}-32 b_{1}+\frac{32}{27} b_{3}-\frac{128}{9} b_{4}+8 m_{0} b_{5}\right. \\
& \left.-\frac{8}{27} m_{0} b_{7}+\frac{32}{9} m_{0} b_{8}\right) I^{2}+\left(-20 b_{0}-\frac{80}{3} b_{D}-\frac{40}{3} b_{F}+40 b_{1}+\frac{40}{9} b_{2}-\frac{40}{27} b_{3}\right. \\
& \left.\left.+\frac{160}{9} b_{4}-10 m_{0} b_{5}-\frac{10}{9} m_{0} b_{6}+\frac{10}{27} m_{0} b_{7}-\frac{40}{9} m_{0} b_{8}\right) I\right) \sin (2 \varepsilon), \\
& \epsilon_{3, p}^{K}=4 b_{0}+\left(4+\frac{8}{3} D^{2}+\frac{8}{3} D F\right) b_{D}+\left(\frac{4}{3} D^{2}+4 F^{2}\right) b_{F}-4\left(b_{1}+b_{3}+b_{4}\right) \\
& +m_{0}\left(b_{5}+b_{7}+b_{8}\right)+\frac{m_{0}}{2} b_{9}-\left(\left(\frac{16}{9} D^{2}+\frac{16}{3} D F\right) b_{D}+\left(\frac{8}{3} D^{2}+8 F^{2}\right) b_{F}\right) I, \\
& \epsilon_{3, p}^{\pi K}=-\frac{8}{3}\left(D^{2}+D F\right) b_{D}-\left(\frac{4}{3} D^{2}+4 F^{2}\right) b_{F} \\
& +\left(\left(\frac{16}{9} D^{2}+\frac{16}{3} D F\right) b_{D}+\left(\frac{8}{3} D^{2}+8 F^{2}\right) b_{F}\right) I,
\end{aligned}
$$




$$
\begin{aligned}
\epsilon_{3, n}^{K} & =4 b_{0}+\left(2-2 D^{2}+4 D F-2 F^{2}\right)\left(b_{D}-b_{F}\right)+2\left(-b_{1}+b_{2}-b_{3}\right)-4 b_{4} \\
& +\frac{m_{0}}{2}\left(b_{5}-b_{6}+b_{7}\right)+m_{0} b_{8}+\left(4 D^{2}-8 D F+4 F^{2}\right)\left(b_{D}-b_{F}\right) I \\
\epsilon_{3, n}^{\pi K} & =\left(2 D^{2}-4 D F+2 F^{2}\right)\left(b_{D}-b_{F}\right)+\left(-4 D^{2}+8 D F-4 F^{2}\right)\left(b_{D}-b_{F}\right) I
\end{aligned}
$$

$$
\begin{aligned}
\epsilon_{4, p}^{\pi} & =16 b_{0}+\left(8-4 D^{2}+8 D F-4 F^{2}\right)\left(b_{D}-b_{F}\right)+8\left(-b_{1}+b_{2}-b_{3}\right)-16 b_{4} \\
& +2 m_{0}\left(b_{5}-b_{6}+b_{7}\right)+4 m_{0} b_{8}+\left(16 b_{0}+8\left(b_{D}-b_{F}-b_{1}+b_{2}-b_{3}\right)-16 b_{4}\right. \\
& \left.+2 m_{0}\left(b_{5}-b_{6}+b_{7}\right)+4 m_{0} b_{8}\right) I^{2}+\left(-32 b_{0}+\left(-16+4 D^{2}-8 D F+4 F^{2}\right)\left(b_{D}-b_{F}\right)\right. \\
& \left.+16\left(b_{1}-b_{2}+b_{3}\right)+32 b_{4}+4 m_{0}\left(-b_{5}+b_{6}-b_{7}\right)-8 m_{0} b_{8}\right) I \\
\epsilon_{4, p}^{K} & =4 b_{0}+\left(2-2 D^{2}+4 D F-2 F^{2}\right)\left(b_{D}-b_{F}\right)+2\left(-b_{1}+b_{2}-b_{3}\right)-4 b_{4} \\
& +\frac{m_{0}}{2}\left(b_{5}-b_{6}+b_{7}\right)+m_{0} b_{8}+\left(16 b_{0}+8\left(b_{D}-b_{F}-b_{1}+b_{2}-b_{3}\right)-16 b_{4}\right. \\
& \left.+2 m_{0}\left(b_{5}-b_{6}+b_{7}\right)+4 m_{0} b_{8}\right) I^{2}+\left(-16 b_{0}+\left(-8+4 D^{2}-8 D F+4 F^{2}\right)\left(b_{D}-b_{F}\right)\right. \\
& \left.+8\left(b_{1}-b_{2}+b_{3}\right)+16 b_{4}+2 m_{0}\left(-b_{5}+b_{6}-b_{7}\right)-4 m_{0} b_{8}\right) I \\
\epsilon_{4, p}^{\pi K} & =-16 b_{0}+\left(-8+6 D^{2}-12 D F+6 F^{2}\right)\left(b_{D}-b_{F}\right)+8\left(b_{1}-b_{2}+b_{3}\right)+16 b_{4} \\
& +2 m_{0}\left(-b_{5}+b_{6}-b_{7}\right)-4 m_{0} b_{8}+\left(-32 b_{0}+16\left(-b_{D}+b_{F}+b_{1}-b_{2}+b_{3}\right)+32 b_{4}\right. \\
& \left.+4 m_{0}\left(-b_{5}+b_{6}-b_{7}\right)-8 m_{0} b_{8}\right) I^{2}+\left(48 b_{0}+\left(24-8 D^{2}+16 D F-8 F^{2}\right)\left(b_{D}-b_{F}\right)\right. \\
& \left.+24\left(-b_{1}+b_{2}-b_{3}\right)-48 b_{4}+6 m_{0}\left(b_{5}-b_{6}+b_{7}\right)+12 m_{0} b_{8}\right) I,
\end{aligned}
$$

$$
\begin{aligned}
\epsilon_{4, n}^{\pi} & =16 b_{0}+\left(16+\frac{16}{3}\left(D^{2}+D F\right)\right) b_{D}+\left(\frac{8}{3} D^{2}+8 F^{2}\right) b_{F}-16\left(b_{1}+b_{3}+b_{4}\right) \\
& +4 m_{0}\left(b_{5}+b_{7}+b_{8}\right)+2 m_{0} b_{9}+\left(16 b_{0}+\left(16+\frac{64}{9} D^{2}\right) b_{D}-16\left(b_{1}+b_{3}+b_{4}\right)\right. \\
& \left.+4 m_{0}\left(b_{5}+b_{7}+b_{8}\right)+2 m_{0} b_{9}\right) I^{2}+\left(-32 b_{0}-\left(32+\frac{112}{9} D^{2}+\frac{16}{3} D F\right) b_{D}\right. \\
& \left.-\left(\frac{8}{3} D^{2}+8 F^{2}\right) b_{F}+32\left(b_{1}+b_{3}+b_{4}\right)-8 m_{0}\left(b_{5}+b_{7}+b_{8}\right)-4 m_{0} b_{9}\right) I \\
\epsilon_{4, n}^{K} & =4 b_{0}+\left(4+\frac{8}{3}\left(D^{2}+D F\right)\right) b_{D}+\left(\frac{4}{3} D^{2}+4 F^{2}\right) b_{F}-4\left(b_{1}+b_{3}+b_{4}\right) \\
& +m_{0}\left(b_{5}+b_{7}+b_{8}\right)+\frac{m_{0}}{2} b_{9}+\left(16 b_{0}+\left(16+\frac{64}{9} D^{2}\right) b_{D}-16\left(b_{1}+b_{3}+b_{4}\right)\right. \\
& \left.+4 m_{0}\left(b_{5}+b_{7}+b_{8}\right)+2 m_{0} b_{9}\right) I^{2}+\left(-16 b_{0}-\left(16+\frac{80}{9} D^{2}+\frac{16}{3} D F\right) b_{D}\right. \\
& \left.-\left(\frac{8}{3} D^{2}+8 F^{2}\right) b_{F}+16\left(b_{1}+b_{3}+b_{4}\right)-4 m_{0}\left(b_{5}+b_{7}+b_{8}\right)-2 m_{0} b_{9}\right) I
\end{aligned}
$$




$$
\begin{aligned}
\epsilon_{4, n}^{\pi K} & =-16 b_{0}-\left(16+8\left(D^{2}+D F\right)\right) b_{D}-\left(4 D^{2}+12 F^{2}\right) b_{F}+16\left(b_{1}+b_{3}+b_{4}\right) \\
& -4 m_{0}\left(b_{5}+b_{7}+b_{8}\right)-2 m_{0} b_{9}+\left(-32 b_{0}-\left(32+\frac{128}{9} D^{2}\right) b_{D}+32\left(b_{1}+b_{3}+b_{4}\right)\right. \\
& \left.-8 m_{0}\left(b_{5}+b_{7}+b_{8}\right)-4 m_{0} b_{9}\right) I^{2}+\left(48 b_{0}+\left(48+\frac{64}{3} D^{2}+\frac{32}{3} D F\right) b_{D}\right. \\
& \left.+\left(\frac{16}{3} D^{2}+16 F^{2}\right) b_{F}-48\left(b_{1}+b_{3}+b_{4}\right)+12 m_{0}\left(b_{5}+b_{7}+b_{8}\right)+6 m_{0} b_{9}\right) I
\end{aligned}
$$

$$
\begin{aligned}
\epsilon_{5, p}^{\pi} & =\frac{17}{3} b_{0}+\frac{2}{3} b_{D}-\frac{4}{3} b_{F}-\frac{2}{3} b_{1}+\frac{4}{3} b_{2}-\frac{26}{9} b_{3}-\frac{14}{3} b_{4}+\frac{m_{0}}{6} b_{5}-\frac{m_{0}}{3} b_{6}+\frac{13}{18} m_{0} b_{7}+\frac{7}{6} m_{0} b_{8} \\
& +\left(4 b_{0}+\frac{8}{3}\left(b_{D}-b_{1}\right)+\frac{16}{9} b_{2}-\frac{56}{27} b_{3}-\frac{40}{9} b_{4}+\frac{2}{3} m_{0} b_{5}-\frac{4}{9} m_{0} b_{6}+\frac{14}{27} m_{0} b_{7}+\frac{10}{9} m_{0} b_{8}\right) I^{2} \\
& +\left(-8 b_{0}+\frac{8}{3}\left(-b_{D}+b_{F}+b_{1}\right)-\frac{32}{9} b_{2}+\frac{40}{9} b_{3}+8 b_{4}-\frac{2}{3} m_{0} b_{5}+\frac{8}{9} m_{0} b_{6}-\frac{10}{9} m_{0} b_{7}\right. \\
& \left.-2 m_{0} b_{8}\right) I+\left(-\frac{4}{3} b_{0}-\frac{1}{3} b_{D}-\frac{5}{3} b_{F}+\frac{1}{3} b_{1}+\frac{5}{9} b_{2}+\frac{13}{27} b_{3}+\frac{8}{9} b_{4}-\frac{m_{0}}{12} b_{5}-\frac{5}{36} m_{0} b_{6}\right. \\
& \left.-\frac{13}{108} m_{0} b_{7}-\frac{2}{9} m_{0} b_{8}\right) \cos (2 \varepsilon)+\frac{1}{\sqrt{3}}\left(10 b_{0}+b_{D}-3 b_{F}-b_{1}+\frac{7}{3} b_{2}-5 b_{3}-8 b_{4}\right. \\
& +\frac{m_{0}}{4} b_{5}-\frac{7}{12} m_{0} b_{6}+\frac{5}{4} m_{0} b_{7}+2 m_{0} b_{8}+\left(8 b_{0}+\frac{8}{3} b_{D}-8 b_{F}-\frac{8}{3} b_{1}+\frac{40}{9} b_{2}-\frac{104}{27} b_{3}\right. \\
& \left.-\frac{64}{9} b_{4}+\frac{2}{3} m_{0} b_{5}-\frac{10}{9} m_{0} b_{6}+\frac{26}{27} m_{0} b_{7}+\frac{16}{9} m_{0} b_{8}\right) I^{2}+\left(-16 b_{0}-4 b_{D}+\frac{20}{3} b_{F}\right. \\
& \left.\left.+4 b_{1}-\frac{52}{9} b_{2}+\frac{220}{27} b_{3}+\frac{128}{9} b_{4}-m_{0} b_{5}+\frac{13}{9} m_{0} b_{6}-\frac{55}{27} m_{0} b_{7}-\frac{32}{9} m_{0} b_{8}\right) I\right) \sin (2 \varepsilon),
\end{aligned}
$$

$$
\begin{aligned}
\epsilon_{5, p}^{K} & =\frac{8}{3}\left(b_{0}+b_{D}+b_{F}-b_{1}\right)-\frac{8}{9} b_{3}-\frac{8}{3} b_{4}+\frac{2}{3} m_{0} b_{5}+\frac{2}{9} m_{0} b_{7}+\frac{2}{3} m_{0} b_{8}+\left(4 b_{0}\right. \\
& \left.+\frac{8}{3}\left(b_{D}-b_{1}\right)+\frac{16}{9} b_{2}-\frac{56}{27} b_{3}-\frac{40}{9} b_{4}+\frac{2}{3} m_{0} b_{5}-\frac{4}{9} m_{0} b_{6}+\frac{14}{27} m_{0} b_{7}+\frac{10}{9} m_{0} b_{8}\right) I^{2} \\
& +\left(-4 b_{0}-\frac{8}{3} b_{D}-\frac{16}{3} b_{F}+\frac{8}{3} b_{1}-\frac{8}{9} b_{2}+\frac{8}{3} b_{3}+\frac{16}{3} b_{4}-\frac{2}{3} m_{0} b_{5}+\frac{2}{9} m_{0} b_{6}-\frac{2}{3} m_{0} b_{7}\right. \\
& \left.-\frac{4}{3} m_{0} b_{8}\right) I+\left(\frac{8}{3}\left(b_{0}+b_{D}-b_{F}-b_{1}\right)+\frac{8}{9} b_{2}-\frac{8}{27} b_{3}-\frac{16}{9} b_{4}+\frac{2}{3} m_{0} b_{5}-\frac{2}{9} m_{0} b_{6}\right. \\
& \left.+\frac{2}{27} m_{0} b_{7}+\frac{4}{9} m_{0} b_{8}\right) \cos (2 \varepsilon)+\frac{1}{\sqrt{3}}\left(4\left(-b_{D}-b_{F}+b_{1}\right)+\frac{4}{3}\left(b_{2}-b_{3}\right)-m_{0} b_{5}\right. \\
& +\frac{m_{0}}{3}\left(-b_{6}+b_{7}\right)+\left(8 b_{0}+\frac{8}{3} b_{D}-8 b_{F}-\frac{8}{3} b_{1}+\frac{40}{9} b_{2}-\frac{104}{27} b_{3}-\frac{64}{9} b_{4}+\frac{2}{3} m_{0} b_{5}\right. \\
& \left.-\frac{10}{9} m_{0} b_{6}+\frac{26}{27} m_{0} b_{7}+\frac{16}{9} m_{0} b_{8}\right) I^{2}+\left(4\left(-b_{0}+b_{D}\right)+\frac{20}{3} b_{F}-4 b_{1}-\frac{28}{9} b_{2}+\frac{100}{27} b_{3}\right. \\
& \left.\left.+\frac{32}{9} b_{4}+m_{0} b_{5}+\frac{7}{9} m_{0} b_{6}-\frac{25}{27} m_{0} b_{7}-\frac{8}{9} m_{0} b_{8}\right) I\right) \sin (2 \varepsilon),
\end{aligned}
$$




$$
\begin{aligned}
& \epsilon_{5, p}^{\pi K}=-\frac{16}{3} b_{0}+\frac{4}{3}\left(-b_{D}-b_{F}+b_{1}-b_{2}\right)+\frac{28}{9} b_{3}+\frac{16}{3} b_{4}+\frac{m_{0}}{3}\left(-b_{5}+b_{6}\right)-\frac{7}{9} m_{0} b_{7} \\
& -\frac{4}{3} m_{0} b_{8}+\left(-8 b_{0}+\frac{16}{3}\left(-b_{D}+b_{1}\right)-\frac{32}{9} b_{2}+\frac{112}{27} b_{3}+\frac{80}{9} b_{4}-\frac{4}{3} m_{0} b_{5}+\frac{8}{9} m_{0} b_{6}\right. \\
& \left.-\frac{28}{27} m_{0} b_{7}-\frac{20}{9} m_{0} b_{8}\right) I^{2}+\left(12 b_{0}+\frac{16}{3} b_{D}+\frac{8}{3} b_{F}-\frac{16}{3} b_{1}+\frac{40}{9} b_{2}-\frac{64}{9} b_{3}-\frac{40}{3} b_{4}\right. \\
& \left.+\frac{4}{3} m_{0} b_{5}-\frac{10}{9} m_{0} b_{6}+\frac{16}{9} m_{0} b_{7}+\frac{10}{3} m_{0} b_{8}\right) I+\left(\frac{4}{3}\left(-b_{0}-b_{D}+b_{F}+b_{1}\right)-\frac{4}{9} b_{2}\right. \\
& \left.+\frac{4}{27} b_{3}+\frac{8}{9} b_{4}-\frac{m_{0}}{3} b_{5}+\frac{m_{0}}{9} b_{6}-\frac{m_{0}}{27} b_{7}-\frac{2}{9} m_{0} b_{8}\right) \cos (2 \varepsilon) \\
& +\frac{1}{\sqrt{3}}\left(-10 b_{0}+4 b_{F}-\frac{8}{3} b_{2}+\frac{16}{3} b_{3}+8 b_{4}+\frac{2}{3} m_{0} b_{6}-\frac{4}{3} m_{0} b_{7}-2 m_{0} b_{8}\right. \\
& +\left(-16 b_{0}-\frac{16}{3} b_{D}+16 b_{F}+\frac{16}{3} b_{1}-\frac{80}{9} b_{2}+\frac{208}{27} b_{3}+\frac{128}{9} b_{4}-\frac{4}{3} m_{0} b_{5}+\frac{20}{9} m_{0} b_{6}\right. \\
& \left.-\frac{52}{27} m_{0} b_{7}-\frac{32}{9} m_{0} b_{8}\right) I^{2}+\left(20 b_{0}-\frac{40}{3} b_{F}+\frac{80}{9} b_{2}-\frac{320}{27} b_{3}-\frac{160}{9} b_{4}-\frac{20}{9} m_{0} b_{6}\right. \\
& \left.\left.+\frac{80}{27} m_{0} b_{7}+\frac{40}{9} m_{0} b_{8}\right) I\right) \sin (2 \varepsilon) \\
& \epsilon_{5, n}^{\pi}=\frac{17}{3} b_{0}+6 b_{D}+4 b_{F}-\frac{26}{3} b_{1}-\frac{4}{3} b_{2}-\frac{2}{9} b_{3}-\frac{14}{3} b_{4}+\frac{13}{6} m_{0} b_{5}+\frac{m_{0}}{3} b_{6}+\frac{m_{0}}{18} b_{7} \\
& +\frac{7}{6} m_{0} b_{8}+\left(4 b_{0}+\frac{8}{3} b_{D}-8 b_{1}-\frac{8}{27} b_{3}-\frac{40}{9} b_{4}+2 m_{0} b_{5}+\frac{2}{27} m_{0} b_{7}+\frac{10}{9} m_{0} b_{8}\right) I^{2} \\
& +\left(-8\left(b_{0}+b_{D}\right)-\frac{8}{3} b_{F}+16 b_{1}+\frac{8}{9} b_{2}+8 b_{4}-4 m_{0} b_{5}-\frac{2}{9} m_{0} b_{6}-2 m_{0} b_{8}\right) I \\
& +\left(-\frac{4}{3} b_{0}-\frac{1}{3} b_{D}-\frac{5}{3} b_{F}+\frac{1}{3} b_{1}+\frac{5}{9} b_{2}+\frac{13}{27} b_{3}+\frac{8}{9} b_{4}-\frac{m_{0}}{12} b_{5}-\frac{5}{36} m_{0} b_{6}-\frac{13}{108} m_{0} b_{7}\right. \\
& \left.-\frac{2}{9} m_{0} b_{8}\right) \cos (2 \varepsilon)+\frac{1}{\sqrt{3}}\left(10 b_{0}+11 b_{D}+7 b_{F}-15 b_{1}-\frac{7}{3} b_{2}-\frac{1}{3} b_{3}-8 b_{4}\right. \\
& +\frac{15}{4} m_{0} b_{5}+\frac{7}{12} m_{0} b_{6}+\frac{m_{0}}{12} b_{7}+2 m_{0} b_{8}+\left(8 b_{0}+\frac{32}{3} b_{D}-16 b_{1}+\frac{16}{27} b_{3}\right. \\
& \left.-\frac{64}{9} b_{4}+4 m_{0} b_{5}-\frac{4}{27} m_{0} b_{7}+\frac{16}{9} m_{0} b_{8}\right) I^{2}+\left(-16 b_{0}-\frac{52}{3} b_{D}-\frac{20}{3} b_{F}+28 b_{1}\right. \\
& \left.\left.+\frac{20}{9} b_{2}+\frac{4}{27} b_{3}+\frac{128}{9} b_{4}-7 m_{0} b_{5}-\frac{5}{9} m_{0} b_{6}-\frac{m_{0}}{27} b_{7}-\frac{32}{9} m_{0} b_{8}\right) I\right) \sin (2 \varepsilon),
\end{aligned}
$$




$$
\begin{aligned}
\epsilon_{5, n}^{K} & =\frac{8}{3}\left(b_{0}-b_{1}\right)-\frac{8}{9} b_{3}-\frac{8}{3} b_{4}+\frac{2}{3} m_{0} b_{5}+\frac{2}{9} m_{0} b_{7}+\frac{2}{3} m_{0} b_{8}+\left(4 b_{0}+\frac{8}{3} b_{D}-8 b_{1}-\frac{8}{27} b_{3}\right. \\
& \left.-\frac{40}{9} b_{4}+2 m_{0} b_{5}+\frac{2}{27} m_{0} b_{7}+\frac{10}{9} m_{0} b_{8}\right) I^{2}+\left(-4 b_{0}-\frac{8}{3} b_{F}+8 b_{1}+\frac{8}{9}\left(b_{2}+b_{3}\right)\right. \\
& \left.+\frac{16}{3} b_{4}-2 m_{0} b_{5}-\frac{2}{9} m_{0}\left(b_{6}+b_{7}\right)-\frac{4}{3} m_{0} b_{8}\right) I+\left(\frac{8}{3}\left(b_{0}+b_{D}-b_{F}-b_{1}\right)+\frac{8}{9} b_{2}\right. \\
& \left.-\frac{8}{27} b_{3}-\frac{16}{9} b_{4}+\frac{2}{3} m_{0} b_{5}-\frac{2}{9} m_{0} b_{6}+\frac{2}{27} m_{0} b_{7}+\frac{4}{9} m_{0} b_{8}\right) \cos (2 \varepsilon) \\
& +\frac{1}{\sqrt{3}}\left(4\left(b_{D}+b_{F}-b_{1}\right)+\frac{4}{3}\left(-b_{2}+b_{3}\right)+m_{0} b_{5}+\frac{m_{0}}{3}\left(b_{6}-b_{7}\right)\right. \\
& +\left(8 b_{0}+\frac{32}{3} b_{D}-16 b_{1}+\frac{16}{27} b_{3}-\frac{64}{9} b_{4}+4 m_{0} b_{5}-\frac{4}{27} m_{0} b_{7}+\frac{16}{9} m_{0} b_{8}\right) I^{2} \\
& +\left(-4 b_{0}-\frac{28}{3} b_{D}-\frac{20}{3} b_{F}+12 b_{1}+\frac{20}{9} b_{2}-\frac{44}{27} b_{3}+\frac{32}{9} b_{4}-3 m_{0} b_{5}-\frac{5}{9} m_{0} b_{6}\right. \\
& \left.\left.+\frac{11}{27} m_{0} b_{7}-\frac{8}{9} m_{0} b_{8}\right) I\right) \sin (2 \varepsilon),
\end{aligned}
$$

$$
\begin{aligned}
\epsilon_{5, n}^{\pi K} & =-\frac{16}{3} b_{0}-4\left(b_{D}+b_{F}\right)+\frac{28}{3} b_{1}+\frac{4}{3} b_{2}+\frac{4}{9} b_{3}+\frac{16}{3} b_{4}-\frac{7}{3} m_{0} b_{5}-\frac{m_{0}}{3} b_{6}-\frac{m_{0}}{9} b_{7} \\
& -\frac{4}{3} m_{0} b_{8}+\left(-8 b_{0}-\frac{16}{3} b_{D}+16 b_{1}+\frac{16}{27} b_{3}+\frac{80}{9} b_{4}-4 m_{0} b_{5}-\frac{4}{27} m_{0} b_{7}-\frac{20}{9} m_{0} b_{8}\right) I^{2} \\
& +\left(12 b_{0}+8 b_{D}+\frac{16}{3} b_{F}-24 b_{1}-\frac{16}{9} b_{2}-\frac{8}{9} b_{3}-\frac{40}{3} b_{4}+6 m_{0} b_{5}+\frac{4}{9} m_{0} b_{6}+\frac{2}{9} m_{0} b_{7}\right. \\
& \left.+\frac{10}{3} m_{0} b_{8}\right) I+\left(\frac{4}{3}\left(-b_{0}-b_{D}+b_{F}+b_{1}\right)-\frac{4}{9} b_{2}+\frac{4}{27} b_{3}+\frac{8}{9} b_{4}-\frac{m_{0}}{3} b_{5}+\frac{m_{0}}{9} b_{6}\right. \\
& \left.-\frac{m_{0}}{27} b_{7}-\frac{2}{9} m_{0} b_{8}\right) \cos (2 \varepsilon)+\frac{1}{\sqrt{3}}\left(-10 b_{0}-12 b_{D}-8 b_{F}+16 b_{1}+\frac{8}{3} b_{2}+8 b_{4}\right. \\
& -4 m_{0} b_{5}-\frac{2}{3} m_{0} b_{6}-2 m_{0} b_{8}+\left(-16 b_{0}-\frac{64}{3} b_{D}+32 b_{1}-\frac{32}{27} b_{3}+\frac{128}{9} b_{4}-8 m_{0} b_{5}\right. \\
& \left.+\frac{8}{27} m_{0} b_{7}-\frac{32}{9} m_{0} b_{8}\right) I^{2}+\left(20 b_{0}+\frac{80}{3} b_{D}+\frac{40}{3} b_{F}-40 b_{1}-\frac{40}{9} b_{2}+\frac{40}{27} b_{3}\right. \\
& \left.\left.-\frac{160}{9} b_{4}+10 m_{0} b_{5}+\frac{10}{9} m_{0} b_{6}-\frac{10}{27} m_{0} b_{7}+\frac{40}{9} m_{0} b_{8}\right) I\right) \sin (2 \varepsilon),
\end{aligned}
$$

$$
\begin{aligned}
& \epsilon_{6, p}^{\pi}=\left(16 D^{2}+32 D F+16 F^{2}\right)\left(b_{D}+b_{F}\right)(1-I), \\
& \epsilon_{6, p}^{\pi K}=\left(16 D^{2}+32 D F+16 F^{2}\right)\left(b_{D}+b_{F}\right)(-1+I), \\
& \epsilon_{6, n}^{\pi}=\left(16 D^{2}+32 D F+16 F^{2}\right)\left(b_{D}+b_{F}\right)(-1+I), \\
& \epsilon_{6, n}^{\pi K}=\left(16 D^{2}+32 D F+16 F^{2}\right)\left(b_{D}+b_{F}\right)(1-I),
\end{aligned}
$$




$$
\begin{aligned}
\epsilon_{8, p}^{K} & =-\frac{32}{3}\left(D^{2}+D F\right) b_{D}-\left(\frac{16}{3} D^{2}+16 F^{2}\right) b_{F} \\
& +\left(\left(\frac{64}{9} D^{2}+\frac{64}{3} D F\right) b_{D}+\left(\frac{32}{3} D^{2}+32 F^{2}\right) b_{F}\right) I \\
\epsilon_{8, p}^{\pi K} & =\frac{32}{3}\left(D^{2}+D F\right) b_{D}+\left(\frac{16}{3} D^{2}+16 F^{2}\right) b_{F} \\
& -\left(\left(\frac{64}{9} D^{2}+\frac{64}{3} D F\right) b_{D}+\left(\frac{32}{3} D^{2}+32 F^{2}\right) b_{F}\right) I
\end{aligned}
$$

$$
\begin{aligned}
& \epsilon_{8, n}^{K}=\left(8 D^{2}-16 D F+8 F^{2}\right)\left(b_{D}-b_{F}\right)+\left(-16 D^{2}+32 D F-16 F^{2}\right)\left(b_{D}-b_{F}\right) I \\
& \epsilon_{8, n}^{\pi K}=\left(-8 D^{2}+16 D F-8 F^{2}\right)\left(b_{D}-b_{F}\right)+\left(16 D^{2}-32 D F+16 F^{2}\right)\left(b_{D}-b_{F}\right) I
\end{aligned}
$$

$$
\begin{aligned}
\epsilon_{9, p}^{\pi} & =\left(16 D^{2}-32 D F+16 F^{2}\right)\left(b_{D}-b_{F}\right)(1-I) \\
\epsilon_{9, p}^{K} & =\left(8 D^{2}-16 D F+8 F^{2}\right)\left(b_{D}-b_{F}\right)+\left(-16 D^{2}+32 D F-16 F^{2}\right)\left(b_{D}-b_{F}\right) I, \\
\epsilon_{9, p}^{\pi K} & =\left(-24 D^{2}+48 D F-24 F^{2}\right)\left(b_{D}-b_{F}\right)+\left(32 D^{2}-64 D F+32 F^{2}\right)\left(b_{D}-b_{F}\right) I
\end{aligned}
$$

$$
\begin{aligned}
\epsilon_{9, n}^{\pi} & =-\frac{64}{3}\left(D^{2}+D F\right) b_{D}-\left(\frac{32}{3} D^{2}+32 F^{2}\right) b_{F}-\frac{256}{9} D^{2} b_{D} I^{2} \\
& +\left(\left(\frac{448}{9} D^{2}+\frac{64}{3} D F\right) b_{D}+\left(\frac{32}{3} D^{2}+32 F^{2}\right) b_{F}\right) I, \\
\epsilon_{9, n}^{K} & =-\frac{32}{3}\left(D^{2}+D F\right) b_{D}-\left(\frac{16}{3} D^{2}+16 F^{2}\right) b_{F}-\frac{256}{9} D^{2} b_{D} I^{2} \\
& +\left(\left(\frac{320}{9} D^{2}+\frac{64}{3} D F\right) b_{D}+\left(\frac{32}{3} D^{2}+32 F^{2}\right) b_{F}\right) I, \\
\epsilon_{9, n}^{\pi K} & =32\left(D^{2}+D F\right) b_{D}+\left(16 D^{2}+48 F^{2}\right) b_{F}+\frac{512}{9} D^{2} b_{D} I^{2} \\
& -\left(\left(\frac{256}{3} D^{2}+\frac{128}{3} D F\right) b_{D}+\left(\frac{64}{3} D^{2}+64 F^{2}\right) b_{F}\right) I,
\end{aligned}
$$




$$
\begin{aligned}
\epsilon_{11, p}^{\pi} & =\left(-144 L_{4}-48 L_{5}+288 L_{6}+96 L_{8}\right) \frac{b_{0}}{F_{\pi}^{2}}+\left(96 L_{4}+32 L_{5}-192 L_{6}-64 L_{8}\right) \frac{b_{F}}{F_{\pi}^{2}} \\
& +\frac{m_{0}}{(4 \pi)^{2} F_{\pi}^{2}}\left(-\frac{17}{12} b_{5}+\frac{13}{12} b_{6}-\frac{71}{36} b_{7}-\frac{11}{3} b_{8}\right)-16 d_{1}+24 d_{5}-36 d_{6}-44 d_{7} \\
& +\left(\left(-64 L_{4}+128 L_{6}\right) \frac{b_{0}}{F_{\pi}^{2}}+\left(128 L_{4}-256 L_{6}\right) \frac{b_{F}}{F_{\pi}^{2}}\right. \\
& \left.+\frac{m_{0}}{(4 \pi)^{2} F_{\pi}^{2}}\left(-\frac{5}{3} b_{5}+\frac{13}{9} b_{6}-\frac{41}{27} b_{7}-\frac{28}{9} b_{8}\right)-64 d_{1}+32 d_{5}-16 d_{6}-48 d_{7}\right) I^{2} \\
& +\left(\left(192 L_{4}+32 L_{5}-384 L_{6}-64 L_{8}\right) \frac{b_{0}}{F_{\pi}^{2}}+\left(-256 L_{4}-64 L_{5}+512 L_{6}+128 L_{8}\right) \frac{b_{F}}{F_{\pi}^{2}}\right. \\
& \left.+\frac{m_{0}}{(4 \pi)^{2} F_{\pi}^{2}}\left(\frac{8}{3} b_{5}-\frac{26}{9} b_{6}+\frac{28}{9} b_{7}+6 b_{8}\right)+64\left(d_{1}-d_{5}\right)+48 d_{6}+80 d_{7}\right) I,
\end{aligned}
$$

$$
\begin{aligned}
\epsilon_{11, p}^{K} & =\left(-32 L_{5}+64 L_{8}\right)\left(\frac{b_{D}}{F_{\pi}^{2}}+\frac{b_{F}}{F_{\pi}^{2}}\right)+\frac{m_{0}}{(4 \pi)^{2} F_{\pi}^{2}}\left(-\frac{17}{12} b_{5}+\frac{1}{4} b_{6}-\frac{35}{36} b_{7}-\frac{5}{3} b_{8}-\frac{1}{4} b_{9}\right) \\
& -16\left(d_{1}+d_{2}+d_{3}\right)-32 d_{7}+\left(\left(-64 L_{4}+128 L_{6}\right) \frac{b_{0}}{F_{\pi}^{2}}+\left(128 L_{4}-256 L_{6}\right) \frac{b_{F}}{F_{\pi}^{2}}\right. \\
& \left.+\frac{m_{0}}{(4 \pi)^{2} F_{\pi}^{2}}\left(-\frac{5}{3} b_{5}+\frac{13}{9} b_{6}-\frac{41}{27} b_{7}-\frac{28}{9} b_{8}\right)-64 d_{1}+32 d_{5}-16 d_{6}-48 d_{7}\right) I^{2} \\
& +\left(\left(-32 L_{5}+64 L_{8}\right) \frac{b_{0}}{F_{\pi}^{2}}+\left(-64 L_{4}+128 L_{6}\right) \frac{b_{D}}{F_{\pi}^{2}}\right. \\
& +\left(64\left(-L_{4}+L_{5}\right)+128\left(L_{6}-L_{8}\right)\right) \frac{b_{F}}{F_{\pi}^{2}}+\frac{m_{0}}{(4 \pi)^{2} F_{\pi}^{2}}\left(\frac{5}{3} b_{5}-\frac{11}{9} b_{6}+\frac{5}{3} b_{7}+\frac{10}{3} b_{8}\right) \\
& \left.+64 d_{1}+32 d_{2}-16 d_{5}+64 d_{7}\right) I,
\end{aligned}
$$

$$
\begin{aligned}
\epsilon_{11, p}^{\pi K} & =\left(-96 L_{4}+192 L_{6}\right)\left(\frac{b_{D}}{F_{\pi}^{2}}+\frac{b_{F}}{F_{\pi}^{2}}\right)+\frac{m_{0}}{(4 \pi)^{2} F_{\pi}^{2}}\left(\frac{4}{3}\left(b_{5}-b_{6}\right)+\frac{16}{9} b_{7}+\frac{10}{3} b_{8}\right) \\
& +32 d_{1}+16 d_{2}-24 d_{5}+64 d_{7}+\left(\left(128 L_{4}-256 L_{6}\right) \frac{b_{0}}{F_{\pi}^{2}}+\left(-256 L_{4}+512 L_{6}\right) \frac{b_{F}}{F_{\pi}^{2}}\right. \\
& \left.+\frac{m_{0}}{(4 \pi)^{2} F_{\pi}^{2}}\left(\frac{10}{3} b_{5}-\frac{26}{9} b_{6}+\frac{82}{27} b_{7}+\frac{56}{9} b_{8}\right)+128 d_{1}-64 d_{5}+32 d_{6}+96 d_{7}\right) I^{2} \\
& +\left(\left(-192 L_{4}+384 L_{6}\right) \frac{b_{0}}{F_{\pi}^{2}}+\left(64 L_{4}-128 L_{6}\right) \frac{b_{D}}{F_{\pi}^{2}}+\left(320 L_{4}-640 L_{6}\right) \frac{b_{F}}{F_{\pi}^{2}}\right. \\
& +\frac{m_{0}}{(4 \pi)^{2} F_{\pi}^{2}}\left(-\frac{13}{3} b_{5}+\frac{37}{9} b_{6}-\frac{43}{9} b_{7}-\frac{28}{3} b_{8}\right)-128 d_{1}-32 d_{2}+80 d_{5}-48 d_{6} \\
& \left.-144 d_{7}\right) I
\end{aligned}
$$




$$
\begin{aligned}
\epsilon_{11, n}^{\pi} & =\left(-144 L_{4}-48 L_{5}+288 L_{6}+96 L_{8}\right) \frac{b_{0}}{F_{\pi}^{2}}+\left(-192 L_{4}-64 L_{5}+384 L_{6}+128 L_{8}\right) \frac{b_{D}}{F_{\pi}^{2}} \\
& +\left(-96 L_{4}-32 L_{5}+192 L_{6}+64 L_{8}\right) \frac{b_{F}}{F_{\pi}^{2}}-\frac{m_{0}}{(4 \pi)^{2} F_{\pi}^{2}}\left(\frac{53}{12} b_{5}+\frac{7}{12} b_{6}+\frac{83}{36} b_{7}+\frac{11}{3} b_{8}\right. \\
& \left.+b_{9}\right)-16 d_{1}-32 d_{2}-64 d_{3}-24 d_{5}-36 d_{6}-44 d_{7}+\left(\left(-64 L_{4}+128 L_{6}\right) \frac{b_{0}}{F_{\pi}^{2}}\right. \\
& +\left(-128 L_{4}+256 L_{6}\right) \frac{b_{D}}{F_{\pi}^{2}}-\frac{m_{0}}{(4 \pi)^{2} F_{\pi}^{2}}\left(4 b_{5}+\frac{56}{27} b_{7}+\frac{28}{9} b_{8}+b_{9}\right)-64 d_{3}-16 d_{6} \\
& \left.-48 d_{7}\right) I^{2}+\left(\left(192 L_{4}+32 L_{5}-384 L_{6}-64 L_{8}\right) \frac{b_{0}}{F_{\pi}^{2}}+\left(320 L_{4}+64 L_{5}-640 L_{6}\right.\right. \\
& \left.-128 L_{8}\right) \frac{b_{D}}{F_{\pi}^{2}}+\left(64 L_{4}-128 L_{6}\right) \frac{b_{F}}{F_{\pi}^{2}}+\frac{m_{0}}{(4 \pi)^{2} F_{\pi}^{2}}\left(8 b_{5}+\frac{2}{9} b_{6}+4 b_{7}+6 b_{8}+2 b_{9}\right) \\
& \left.+32 d_{2}+128 d_{3}+16 d_{5}+48 d_{6}+80 d_{7}\right) I,
\end{aligned}
$$

$$
\begin{aligned}
\epsilon_{11, n}^{K} & =\left(32 L_{5}-64 L_{8}\right)\left(\frac{b_{D}}{F_{\pi}^{2}}+\frac{b_{F}}{F_{\pi}^{2}}\right)+\frac{m_{0}}{(4 \pi)^{2} F_{\pi}^{2}}\left(-\frac{17}{12} b_{5}+\frac{1}{4} b_{6}-\frac{35}{36} b_{7}-\frac{5}{3} b_{8}-\frac{1}{4} b_{9}\right) \\
& -16\left(d_{1}+d_{2}+d_{3}\right)-32 d_{7}+\left(\left(-64 L_{4}+128 L_{6}\right) \frac{b_{0}}{F_{\pi}^{2}}+\left(-128 L_{4}+256 L_{6}\right) \frac{b_{D}}{F_{\pi}^{2}}\right. \\
& \left.-\frac{m_{0}}{(4 \pi)^{2} F_{\pi}^{2}}\left(4 b_{5}+\frac{56}{27} b_{7}+\frac{28}{9} b_{8}+b_{9}\right)-64 d_{3}-16 d_{6}-48 d_{7}\right) I^{2} \\
& +\left(\left(-32 L_{5}+64 L_{8}\right) \frac{b_{0}}{F_{\pi}^{2}}+\left(64\left(L_{4}-L_{5}\right)+128\left(-L_{6}+L_{8}\right)\right) \frac{b_{D}}{F_{\pi}^{2}}\right. \\
& +\left(64 L_{4}-128 L_{6}\right) \frac{b_{F}}{F_{\pi}^{2}}+\frac{m_{0}}{(4 \pi)^{2} F_{\pi}^{2}}\left(4 b_{5}+\frac{2}{9} b_{6}+\frac{20}{9} b_{7}+\frac{10}{3} b_{8}+b_{9}\right) \\
& \left.+32 d_{2}+64 d_{3}+16 d_{5}+64 d_{7}\right) I,
\end{aligned}
$$

$$
\begin{aligned}
\epsilon_{11, n}^{\pi K} & =\left(96 L_{4}-192 L_{6}\right)\left(\frac{b_{D}}{F_{\pi}^{2}}+\frac{b_{F}}{F_{\pi}^{2}}\right)+\frac{m_{0}}{(4 \pi)^{2} F_{\pi}^{2}}\left(\frac{13}{3} b_{5}+\frac{1}{3} b_{6}+\frac{19}{9} b_{7}+\frac{10}{3} b_{8}+b_{9}\right) \\
& +32 d_{1}+48 d_{2}+64 d_{3}+24 d_{5}+64 d_{7}+\left(\left(128 L_{4}-256 L_{6}\right) \frac{b_{0}}{F_{\pi}^{2}}\right. \\
& +\left(256 L_{4}-512 L_{6}\right) \frac{b_{D}}{F_{\pi}^{2}}+\frac{m_{0}}{(4 \pi)^{2} F_{\pi}^{2}}\left(8 b_{5}+\frac{112}{27} b_{7}+\frac{56}{9} b_{8}+2 b_{9}\right)+128 d_{3} \\
& \left.+32 d_{6}+96 d_{7}\right) I^{2}+\left(\left(-192 L_{4}+384 L_{6}\right) \frac{b_{0}}{F_{\pi}^{2}}+\left(-384 L_{4}+768 L_{6}\right) \frac{b_{D}}{F_{\pi}^{2}}\right. \\
& +\left(-128 L_{4}+256 L_{6}\right) \frac{b_{F}}{F_{\pi}^{2}}-\frac{m_{0}}{(4 \pi)^{2} F_{\pi}^{2}}\left(12 b_{5}+\frac{4}{9} b_{6}+\frac{56}{9} b_{7}+\frac{28}{3} b_{8}+3 b_{9}\right) \\
& \left.-64 d_{2}-192 d_{3}-32 d_{5}-48 d_{6}-144 d_{7}\right) I .
\end{aligned}
$$

For completeness, we also give the fourth order corrections to the meson masses in the form they were used to arrive at the quark mass representations of the baryon masses. More precisely, in the second order baryon mass corrections $m_{B}^{(2)}=\sum_{P} \gamma_{B}^{P} M_{P}^{2}$ we have 
contributions from the meson masses $M_{P}^{2}=\bar{M}_{P}^{2}+M_{P}^{(4)}$, where $M_{P}^{(4)}$ is the fourth-order correction. We remark that only the charged pion and kaon mass appear in the terms $m_{B}^{(2)}$ due to our conventions. The generic form of these corrections is

$$
\begin{aligned}
F_{\pi}^{2} M_{P}^{(4)} & =\left(\alpha_{1, P}^{\pi} M_{\pi^{+}}^{4}+\alpha_{1, P}^{K} M_{K^{+}}^{4}+\alpha_{1, P}^{\pi K} M_{\pi^{+}}^{2} M_{K^{+}}^{2}\right) \bar{\mu}_{\pi^{+}} \\
& +\left(\alpha_{2, P}^{\pi} M_{\pi^{+}}^{4}+\alpha_{2, P}^{K} M_{K^{+}}^{4}+\alpha_{2, P}^{\pi K} M_{\pi^{+}}^{2} M_{K^{+}}^{2}\right) \bar{\mu}_{\pi^{0}} \\
& +\left(\alpha_{3, P}^{\pi} M_{\pi^{+}}^{4}+\alpha_{3, P}^{K} M_{K^{+}}^{4}+\alpha_{3, P}^{\pi K} M_{\pi^{+}}^{2} M_{K^{+}}^{2}\right) \bar{\mu}_{K^{+}} \\
& +\left(\alpha_{4, P}^{\pi} M_{\pi^{+}}^{4}+\alpha_{4, P}^{K} M_{K^{+}}^{4}+\alpha_{4, P}^{\pi K} M_{\pi^{+}}^{2} M_{K^{+}}^{2}\right) \bar{\mu}_{K^{0}} \\
& +\left(\alpha_{5, P}^{\pi} M_{\pi^{+}}^{4}+\alpha_{5, P}^{K} M_{K^{+}}^{4}+\alpha_{5, P}^{\pi K} M_{\pi^{+}}^{2} M_{K^{+}}^{2}\right) \bar{\mu}_{\eta} \\
& +\alpha_{6, P}^{\pi} M_{\pi^{+}}^{4}+\alpha_{6, P}^{K} M_{K^{+}}^{4}+\alpha_{6, P}^{\pi K} M_{\pi^{+}}^{2} M_{K^{+}}^{2} .
\end{aligned}
$$

The non-vanishing coefficients for $P=\pi^{+}, K^{+}$are

$$
\begin{aligned}
\alpha_{2, \pi^{+}}^{\pi} & =\frac{1}{6}+\frac{1}{9} I+\frac{2}{9} \cos (2 \varepsilon)+\frac{1}{\sqrt{3}}\left(-\frac{1}{3}+\frac{2}{9} I\right) \sin (2 \varepsilon), \\
\alpha_{2, \pi^{+}}^{\pi K} & =-\frac{1}{9} I+\frac{1}{9} \cos (2 \varepsilon)+\frac{1}{\sqrt{3}}\left(\frac{1}{3}-\frac{2}{9} I\right) \sin (2 \varepsilon), \\
\alpha_{5, \pi^{+}}^{\pi} & =\frac{1}{6}+\frac{1}{9} I-\frac{2}{9} \cos (2 \varepsilon)+\frac{1}{\sqrt{3}}\left(\frac{1}{3}-\frac{2}{9} I\right) \sin (2 \varepsilon), \\
\alpha_{5, \pi^{+}}^{\pi K} & =-\frac{1}{9} I-\frac{1}{9} \cos (2 \varepsilon)+\frac{1}{\sqrt{3}}\left(-\frac{1}{3}+\frac{2}{9} I\right) \sin (2 \varepsilon), \\
\alpha_{6, \pi^{+}}^{\pi} & =(-24+16 I) L_{4}-8 L_{5}+(48-32 I) L_{6}+16 L_{8}, \\
\alpha_{6, \pi^{+}}^{\pi K} & =-16 I L_{4}+32 I L_{6}, \\
\alpha_{2, K^{+}}^{K} & =\frac{1}{3}-\frac{1}{9} I-\frac{2}{9} \cos (2 \varepsilon)+\frac{1}{\sqrt{3}}\left(\frac{1}{3}-\frac{2}{9} I\right) \sin (2 \varepsilon), \\
\alpha_{2, K^{+}}^{\pi K} & =-\frac{1}{6}+\frac{1}{9} I+\frac{1}{18} \cos (2 \varepsilon)+\frac{1}{\sqrt{3}}\left(\frac{1}{6}+\frac{2}{9} I\right) \sin (2 \varepsilon), \\
\alpha_{5, K^{+}}^{K} & =\frac{1}{3}-\frac{1}{9} I+\frac{2}{9} \cos (2 \varepsilon)+\frac{1}{\sqrt{3}}\left(-\frac{1}{3}+\frac{2}{9} I\right) \sin (2 \varepsilon), \\
\alpha_{5, K^{+}}^{\pi K} & =-\frac{1}{6}+\frac{1}{9} I-\frac{1}{18} \cos (2 \varepsilon)-\frac{1}{\sqrt{3}}\left(\frac{1}{6}+\frac{2}{9} I\right) \sin (2 \varepsilon), \\
\alpha_{6, K^{+}}^{K} & =-16 I L_{4}-8 L_{5}+32 I L_{6}+16 L_{8}, \\
\alpha_{6, K^{+}}^{\pi K} & =(-24+16 I) L_{4}+(48-32 I) L_{6} .
\end{aligned}
$$

We note that in the isospin limit $m_{u}=m_{d}$, these formulas coincide with the ones in the classic paper [28]. We stress again that the $L_{i}$ are the renormalized values taken at the scale $\lambda=m_{0}$ and similarly for the chiral logarithms hidden in the $\bar{\mu}_{P}$. These LECs are usually given at the scale $\lambda=M_{\rho}$, with $M_{\rho}=770 \mathrm{MeV}$ the $\rho$-meson mass. The corresponding $\beta$-functions to evolve the $L_{i}$ from $\lambda=M_{\rho}$ to $\lambda=m_{0}$ are given in [28].

\section{B. Analysis of tapdole graphs}

Here, we give a detailed analysis of the tadpole diagrams. We had already enumerated in Eq. (2.14) the terms in the dimension two Lagrangian that lead to a non-trivial quark mass 
dependence of the baryon masses. Apart from the terms listed there, one also has terms with two covariant derivatives of the form (we stick to the labeling of [18])

$$
\begin{aligned}
\mathcal{L}^{(2,2)}= & \frac{b_{15}}{2}\left(\operatorname{Tr}\left\{\bar{B}\left[u_{\mu},\left[u_{\nu},\left[D^{\mu},\left[D^{\nu}, B\right]\right]\right]\right]\right\}+\operatorname{Tr}\left\{\bar{B}\left[D_{\nu},\left[D_{\mu},\left[u^{\nu},\left[u^{\mu}, B\right]\right]\right]\right]\right\}\right) \\
+ & \frac{b_{16}}{4}\left(\operatorname{Tr}\left\{\bar{B}\left[u_{\mu},\left\{u_{\nu},\left[D^{\mu},\left[D^{\nu}, B\right]\right]\right\}\right]\right\}+\operatorname{Tr}\left\{\bar{B}\left[D_{\nu},\left[D_{\mu},\left\{u^{\nu},\left[u^{\mu}, B\right]\right\}\right]\right]\right\}\right. \\
& +\operatorname{Tr}\left\{\bar{B}\left\{u_{\mu},\left[u_{\nu},\left[D^{\mu},\left[D^{\nu}, B\right]\right]\right]\right\}\right\}+\operatorname{Tr}\left\{\bar{B}\left[D_{\nu},\left[D_{\mu},\left[u^{\nu},\left\{u^{\mu}, B\right\}\right]\right]\right\}\right) \\
+ & \frac{b_{17}}{2}\left(\operatorname{Tr}\left\{\bar{B}\left\{u_{\mu},\left\{u_{\nu},\left[D^{\mu},\left[D^{\nu}, B\right]\right]\right\}\right\}\right\}+\operatorname{Tr}\left\{\bar{B}\left[D_{\nu},\left[D_{\mu},\left\{u^{\nu},\left\{u^{\mu}, B\right\}\right\}\right]\right]\right\}\right) \\
+ & \frac{b_{18}}{2}\left(\operatorname{Tr}\left\{\bar{B} u_{\mu}\right\} \operatorname{Tr}\left\{u_{\nu}\left[D^{\mu},\left[D^{\nu}, B\right]\right]\right\}+\operatorname{Tr}\left\{\bar{B} u_{\nu}\right\} \operatorname{Tr}\left\{\left[D_{\mu},\left[D^{\nu}, u^{\mu} B\right]\right]\right\}\right. \\
& +\operatorname{Tr}\left\{\bar{B}\left[D_{\mu},\left[D_{\nu}, u^{\nu}\right]\right]\right\} \operatorname{Tr}\left\{u^{\mu} B\right\}+\operatorname{Tr}\left\{\bar{B}\left[D_{\mu}, u_{\nu}\right]\right\} \operatorname{Tr}\left\{\left[D^{\nu}, u^{\mu} B\right]\right\} \\
& \left.+\operatorname{Tr}\left\{\bar{B}\left[D_{\nu}, u^{\nu}\right]\right\} \operatorname{Tr}\left\{\left[D_{\mu}, u^{\mu} B\right]\right\}\right) \\
+ & b_{19}\left(\operatorname{Tr}\left\{\bar{B}\left[D_{\mu},\left[D_{\nu}, B\right]\right]\right\} \operatorname{Tr}\left\{u^{\mu} u^{\nu}\right\}+\operatorname{Tr}\left\{\bar{B}\left[D_{\nu}, B\right]\right\} \operatorname{Tr}\left\{\left[D_{\mu}, u^{\mu} u^{\nu}\right]\right\}\right) .(B .1
\end{aligned}
$$

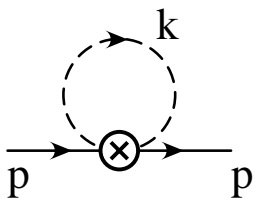

Figure 2: Tadpole graph.

Therefore, the tadpole diagram shown in Fig. 2 with the four-momenta as given there can lead to the following structures (we only display the non-vanishing ones)

$$
\mathcal{A}_{\text {tadpole }} \sim \int_{I} \frac{d^{4} k}{(2 \pi)^{4}}\left\{\mathbf{1}, k_{\mu} k^{\mu}, k_{\mu} k_{\nu} p^{\mu} p^{\nu}, k_{\mu} k_{\nu} p^{\mu} \gamma^{\mu}\right\} \frac{i}{k^{2}-\bar{M}_{P}^{2}}
$$

where the factors $\sim k_{\mu}$ are generated from the chiral building blocks $u_{\mu}$ whereas the factors of $p_{\mu}$ stem from the covariant derivatives $D_{\mu}$ acting on the baryon fields. The terms $\sim \mathbf{1}$ stem from the explicit symmetry breaking $\sim \chi_{+}$ encoded in the terms proportional to $b_{0, D, F}$. Upon integration over the meson momentum in the loop one obtains

$$
\begin{aligned}
\mathcal{A}^{(1)} & =\int_{I} \frac{d^{4} k}{(2 \pi)^{4}} \frac{i}{k^{2}-\bar{M}_{P}^{2}}=\bar{\Delta}_{P}, \\
\mathcal{A}^{(2)} & =\int_{I} \frac{d^{4} k}{(2 \pi)^{4}} \frac{i k^{2}}{k^{2}-\bar{M}_{P}^{2}}=\bar{M}_{P}^{2} \bar{\Delta}_{P}, \\
\mathcal{A}^{(3)} & =\int_{I} \frac{d^{4} k}{(2 \pi)^{4}} p^{\mu} p^{\nu} \frac{i k_{\mu} k_{\nu}}{k^{2}-\bar{M}_{P}^{2}}=p^{2}\left(\frac{1}{4} \bar{M}_{P}^{2} \bar{\Delta}_{P}-\frac{1}{8} \frac{\bar{M}_{P}^{4}}{(4 \pi)^{2}}\right), \\
\mathcal{A}^{(4)} & =\int_{I} \frac{d^{4} k}{(2 \pi)^{4}} \gamma^{\mu} p^{\nu} \frac{i k_{\mu} k_{\nu}}{k^{2}-\bar{M}_{P}^{2}}=\not p\left(\frac{1}{4} \bar{M}_{P}^{2} \bar{\Delta}_{P}-\frac{1}{8} \frac{\bar{M}_{P}^{4}}{(4 \pi)^{2}}\right),
\end{aligned}
$$

with $\bar{\Delta}_{P}$ given in Eq. (3.5). One sees that the terms with one or two derivatives generate quark mass dependent structures $\sim M_{P}^{4}$ that can not be absorbed in the LECs of the terms $\sim u_{\mu} u^{\mu} \sim k_{\mu} k^{\mu}$. However, these additional terms are only of importance if one is interested in varying the quark masses (the procedure of retaining only the dimension two terms $\sim b_{1,2,3,4}$ in [18] for the analysis of the chiral expansion of the baryon masses was thus correct). Furthermore, Eq. (B.3) also shows that the quark mass dependence of the 
terms with one or two covariant derivatives acting on the baryon fields is the same since the diagrams have to be evaluated on the mass shell, $\not p=m_{0}, p^{2}=m_{0}^{2}$.

\section{Renormalization and $\beta$-functions}

Here, we briefly discuss the renormalization of the fourth order loop graphs. We use standard dimensional regularization, the UV infinities are mapped onto simple poles $\sim$ $1 /(d-4)$, where $d$ is the number of space-time dimensions. This leads to the following representation of the fourth order LECs $d_{i}$ in Eq. (2.16)

$$
d_{i}=d_{i}^{r}\left(m_{0}\right)+\frac{\Gamma_{i}}{F_{\pi}^{2}} \bar{L}
$$

with $\bar{L}$ defined in Eq. (3.6) (remember that we work at the scale $\lambda=m_{0}$ ). The corresponding $\beta$-functions are given by

$$
\begin{aligned}
\Gamma_{1}= & -\frac{1}{6} b_{1}+\frac{1}{18} b_{3}+\frac{9}{4} D F b_{F}+\frac{1}{4}\left(\frac{7}{9}+\frac{23}{6} D^{2}+\frac{9}{2} F^{2}\right) b_{D} \\
& +\frac{1}{24 m_{0}}\left(\frac{1}{3} D^{2}-F^{2}\right)+\frac{m_{0}}{24}\left(b_{5}-\frac{1}{3} b_{7}\right), \\
\Gamma_{2}= & \frac{1}{4} b_{2}+\frac{5}{2} D F b_{D}+\frac{1}{4}\left(\frac{1}{3}+5 D^{2}+9 F^{2}\right) b_{F}+\frac{1}{8 m_{0}} D F-\frac{m_{0}}{16} b_{6}, \\
\Gamma_{3}= & -\frac{3}{4} b_{1}-\frac{1}{12} b_{3}+\frac{9}{4} D F b_{F}+\frac{1}{2}\left(1+\frac{13}{4} D^{2}+\frac{9}{4} F^{2}\right) b_{D} \\
& +\frac{1}{16 m_{0}}\left(D^{2}-3 F^{2}\right)+\frac{m_{0}}{16}\left(3 b_{5}+\frac{1}{3} b_{7}+b_{9}\right), \\
\Gamma_{4}= & \frac{3}{2} b_{1}-\frac{1}{18} b_{3}-\left(\frac{11}{18}+4 D^{2}\right) b_{D}+\frac{1}{8 m_{0}}\left(3 F^{2}-D^{2}\right)-\frac{m_{0}}{4}\left(\frac{3}{2} b_{5}-\frac{1}{18} b_{7}+\frac{1}{3} b_{9}\right), \\
\Gamma_{5}= & -\frac{1}{18}\left(13 b_{2}-11 b_{F}\right)-\frac{13}{36}\left(\frac{1}{m_{0}} D F-\frac{1}{2} m_{0} b_{6}\right), \\
\Gamma_{6}= & \frac{11}{18} b_{0}-\frac{1}{4} b_{1}-\frac{35}{108} b_{3}-\frac{11}{18} b_{4}+\frac{1}{3}\left(\frac{11}{12}-D^{2}\right) b_{D}-\frac{1}{16 m_{0}}\left(\frac{35}{27} D^{2}+F^{2}\right) \\
& +\frac{m_{0}}{8}\left(\frac{1}{2} b_{5}+\frac{35}{54} b_{7}+\frac{11}{9} b_{8}\right), \\
& \frac{5}{6} b_{0}-\frac{1}{4} b_{1}-\frac{17}{36} b_{3}-\frac{5}{6} b_{4}-3 D F b_{F}+\frac{1}{2}\left(\frac{7}{18}-\frac{7}{3} D^{2}-3 F^{2}\right) b_{D} \\
\Gamma_{7} & \frac{1}{16 m_{0}}\left(\frac{17}{9} D^{2}+F^{2}\right)+\frac{m_{0}}{8}\left(\frac{1}{2} b_{5}+\frac{17}{18} b_{7}+\frac{5}{3} b_{8}\right) \cdot .
\end{aligned}
$$

These agree with the $\beta$-functions in [18] (if one makes use of Eqs. (5.12)) up to kinetic energy insertions and a few typographical errors in that paper. 


\section{References}

[1] C. Bernard, S. Hashimoto, D. B. Leinweber, P. Lepage, E. Pallante, S. R. Sharpe and

H. Wittig, Nucl. Phys. 119 (Proc. Suppl.) (2003) 170, hep-lat/0209086.

[2] K. Jansen, hep-lat/0311039.

[3] R. D. Young, D. B. Leinweber and A. W. Thomas, Prog. Part. Nucl. Phys. 50 (2003) 399, hep-lat/0212031.

[4] D. B. Leinweber, A. W. Thomas and R. D. Young, hep-lat/0302020.

[5] V. Bernard, T. R. Hemmert and U.-G. Meißner, Nucl. Phys. A 732 (2004) 149, hep-ph/0307115.

[6] M. Procura, T. R. Hemmert and W. Weise, Phys. Rev. D 69 (2004) 034505, hep-lat/0309020.

[7] A. Ali Khan et al. [CP-PACS Collaboration], Phys. Rev. D 65 (2002) 054505, [Erratum-ibid. D 67 (2003) 059901], hep-lat/0105015.

[8] S. Aoki et al. [JLQCD Collaboration], Phys. Rev. D 68 (2003) 054502, hep-lat/0212039.

[9] G. Schierholz et al. [QCDSF-UKQCD Collaboration], in preparation.

[10] A. Ali Khan et al. [QCDSF-UKQCD Collaboration], hep-lat/0312030.

[11] S. R. Beane and M. J. Savage, Nucl. Phys. A 709 (2002) 319, hep-lat/0203003.

[12] K. Kanaya [CP-PACS Collaboration], hep-lat/0310040.

[13] S. Descotes-Genon, L. Girlanda and J. Stern, J. High Energy Phys. 0001 (2000) 041, hep-ph/9910537.

[14] S. R. Beane, hep-lat/0403030.

[15] J. Gasser, Ann. Phys. (NY) 136 (1981) 62.

[16] E. Jenkins, Nucl. Phys. B 368 (1992) 190.

[17] V. Bernard, N. Kaiser and U.-G. Meißner, Z. Physik C 60 (1993) 111, hep-ph/9303311.

[18] B. Borasoy and U.-G. Meißner, Ann. Phys. (NY) 254 (1997) 192, hep-ph/9607432.

[19] S. Steininger, U.-G. Meißner and N. Fettes, J. High Energy Phys. 9809 (1998) 008, hep-ph/9808280.

[20] J. F. Donoghue, B. R. Holstein and B. Borasoy, Phys. Rev. D 59 (1999) 036002, hep-ph/9804281.

[21] J. A. McGovern and M. C. Birse, Phys. Lett. B 446 (1999) 300, hep-ph/9807384.

[22] J. Kambor and M. Mojžiš, J. High Energy Phys. 9904 (1999) 031, hep-ph/9901235.

[23] B. Borasoy, Eur. Phys. J. C 8 (1999) 121, hep-ph/9807453.

[24] M. Mojžiš and J. Kambor, Phys. Lett. B 476 (2000) 344, hep-ph/9912517.

[25] T. Becher and H. Leutwyler, Eur. Phys. J. C 9 (1999) 643, hep-ph/9901384.

[26] P. J. Ellis and K. Torikoshi, Phys. Rev. C 61 (2000) 015205, nucl-th/9904017.

[27] T. Fuchs, J. Gegelia, G. Japaridze and S. Scherer, Phys. Rev. D 68 (2003) 056005, hep-ph/0302117. 
[28] J. Gasser and H. Leutwyler, Nucl. Phys. B 250 (1985) 465.

[29] P. G. Ratcliffe, hep-ph/0402063.

[30] A. Krause, Helv. Phys. Acta 63 (1990) 3.

[31] N. Fettes, U.-G. Meißner, M. Mojžiš and S. Steininger, Ann. Phys. (NY) 283 (2000) 273 [Erratum-ibid. 288 (2001) 249], hep-ph/0001308.

[32] M. Frink and U.-G. Meißner, in preparation.

[33] B. Kubis and U.-G. Meißner, Nucl. Phys. A 679 (2001) 698, hep-ph/0007056.

[34] V. Bernard, N. Kaiser and U.-G. Meißner, Int. J. Mod. Phys. E 4 (1995) 193, hep-ph/9501384

[35] V. Bernard, N. Kaiser and U.-G. Meißner, Nucl. Phys. A 615 (1997) 483, hep-ph/9611253.

[36] N. Fettes, U.-G. Meißner and S. Steininger, Nucl. Phys. A 640 (1998) 199, hep-ph/9803266.

[37] P. Büttiker and U.-G. Meißner, Nucl. Phys. A 668 (2000) 97, hep-ph/9908247.

[38] M. C. M. Rentmeester, R. G. E. Timmermans and J. J. de Swart, Phys. Rev. C 67 (2003) 044001, nucl-th/0302080.

[39] N. Fettes and U.-G. Meißner, Nucl. Phys. A 693 (2001) 693, hep-ph/0101030.

[40] S. Weinberg, Trans. New York Acad. Sci. 38 (1977) 185;

S. Weinberg, in "Chiral Dynamics: Theory and Experiment", A. M. Bernstein and B. R. Holstein (eds.), (Springer Verlag, Berlin, 1995).

[41] R. P. Feynman, Phys. Rev. 56 (1939) 340;

H. Hellmann, Einführung in die Quantenchemie, (Deuticke Verlag, Leipzig, 1937).

[42] U.-G. Meißner and S. Steininger, Phys. Lett. B 419 (1998) 403, hep-ph/9709453.

[43] G. Müller and U.-G. Meißner, Nucl. Phys. B 556 (1999) 265, hep-ph/9903375.

[44] S. Gusken, K. Schilling, R. Sommer, K. H. Mutter and A. Patel, Phys. Lett. B 212 (1988) 216.

[45] R. Altmeyer, M. Göckeler, R. Horsley, E. Laermann and G. Schierholz [MTc Collaboration], hep-lat/9311012.

[46] M. Fukugita, Y. Kuramashi, M. Okawa and A. Ukawa, Phys. Rev. D 51 (1995) 5319, hep-lat/9408002.

[47] S. J. Dong, J. F. Lagae and K. F. Liu, Phys. Rev. D 54 (1996) 5496, hep-ph/9602259.

[48] D. B. Leinweber, A. W. Thomas and S. V. Wright, Phys. Lett. B 482 (2000) 109, hep-lat/0001007. 